\author{
Universidade de Brasília \\ Departamento de Matemática \\ Programa de Pós-Graduação em Matemática
}

\author{
DISSERTAÇÃO
}

\title{
Generalizando um teorema de P. Hall sobre grupos finitos-por-nilpotentes
}

\author{
Aluno: Leandro Araújo Castro \\ Orientadora: Cristina Acciarri
}




\title{
Leandro Araújo Castro ${ }^{1}$
}

\section{Generalizando um teorema de P. Hall sobre grupos finitos-por-nilpotentes}

\begin{abstract}
Dissertação apresentada ao Programa de Pós-Graduação em Matemática do Departamento de Matemática da Universidade de Brasília, em cumprimento às exigências para obtenção do Título de Mestre em Matemática
\end{abstract}

Orientadora: Prof. Cristina Acciarri 


\section{Sumário}

Agradecimentos $\quad$ ii

Resumo $\quad$ iii

Abstract iv

Introdução $\quad$ V

Capítulo 1. Preliminares 1

1.1. Subgrupos finitamente gerados e a condição maximal 2

1.2. Sobre comutadores e centralizadores 9

1.3. As séries centrais ascendente e descendente 14

1.4. Grupos solúveis e nilpotentes 23

1.5. $p$-grupos extra especiais 24

1.6. Ação de grupos $\quad 27$

1.7. O homomorfismo transfer 31

1.8. Produto tensorial de grupos abelianos 34

Capítulo 2. Os Teoremas de Schur, Baer e Hall 37

2.1. Teorema de Schur como aplicação do transfer 37

2.2. Recíprocas do Teorema de Schur 40

2.3. Teorema de Baer 43

2.4. Recíprocas do Teorema de Baer 47

2.5. Teorema de Hall 55

Capítulo 3. Generalizando o Teorema de Hall 64

3.1. Seguindo os passos de P. Hall 65

3.2. Grupos capable e grupos i-capable 79

Índice Remissivo $\quad 85$

Referências Bibliográficas $\quad 86$ 


\section{Agradecimentos}

À minha orientadora Cristina Acciarri, pela disponibilidade, paciência e atenção. A dedicação incansável da professora Cristina foi o que garantiu a qualidade do trabalho e que livrou o texto de incontáveis erros.

À professora Aline Pinto e ao professor Jhone Caldeira, membros da banca examinadora, pelos valiosos comentários e sugestões.

À minha mãe Cândida Araújo, a principal incentivadora dos meus estudos.

À Renata Guedes, pelo carinho e companheirismo e por sempre me encorajar a seguir em frente.

Aos colegas da pós graduação, em especial ao Raimundo Bastos, pela ajuda e pelos valiosos conselhos.

Aos funcionários do departamento de Matemática, em especial à Bruna Ribeiro, pela eficiência e prestatividade.

Aos professores com quem tive aula no mestrado, especialmente o professor Noraí Rocco que me ensinou a gostar de Álgebra e o professor Pavel Shumyatsky com quem pude aprofundar meus conhecimentos em Teoria de Grupos. 


\section{Resumo}

Um conhecido teorema devido a Schur [25, 10.1.4] assegura que, se $G$ é um grupo tal que $[G: Z(G)]$ é finito então $G^{\prime}$ é finito. Baer [2] forneceu uma generalização para todos os termos das séries centrais ascendente e descendente, assegurando que se $\left[G: Z_{i}(G)\right]$ é finito então $\gamma_{i+1}(G)$ é finito. A recíproca do Teorema de Baer não é válida em geral. Não obstante, P. Hall $[\mathbf{1 1}]$ mostrou que se $\gamma_{i+1}(G)$ é finito então $\left[G: Z_{2 i}(G)\right]$ é finito. A presente dissertação tem por base um trabalho de G. Fernández-Alcober e M. Morigi [7] onde mostra-se que a mesma conclusão do Teorema de Hall vale sob a hipótese mais fraca de que $\left[\gamma_{i+1}(G): \gamma_{i+1}(G) \cap Z_{i}(G)\right]$ é finito.

Dado um inteiro $i \geq 1$, dizemos que um grupo $G$ é $i$-capable se $G$ é isomorfo a $H / Z_{i}(H)$, para algum grupo $H$. O resultado de FernándezAlcober e Morigi garante que, para um grupo $i$-capable $G$, vale a recíproca do Teorema de Baer. Além dos resultados de [7], neste trabalho estudamos outras classes de grupos para as quais vale a recíproca do Teorema de Baer. 


\begin{abstract}
A well known theorem due to Schur $[\mathbf{2 5}, 10.1 .4]$ asserts that, if $G$ is a group such that $[G: Z(G)]$ is finite, then $G^{\prime}$ is finite. Baer $[2]$ generalized Schur's result to other terms of the upper and lower central series, proving that if $\left[G: Z_{i}(G)\right]$ is finite, then $\gamma_{i+1}(G)$ is finite. The converse of Baer's Theorem does not hold in general. However, P. Hall $[\mathbf{1 1}]$ showed that if $\gamma_{i+1}(G)$ is finite, then $\left[G: Z_{2 i}(G)\right]$ is finite. This dissertation is based on the work of G. Fernández-Alcober and M. Morigi [7]. The authors showed [7, Theorem A] that the same conclusion of Hall's result is valid under the weaker hypotesis that $\left[\gamma_{i+1}(G): \gamma_{i+1}(G) \cap Z_{i}(G)\right]$ is finite.

Given an integer $i \geq 1$, we say that $G$ is $i$-capable if $G$ is isomorphic to $H / Z_{i}(H)$, for some group $H$. The result of Fernández-Alcober and Morigi ensures that the converse of Baer's Theorem holds for any $i$ capable group $G$. Apart from the results in [7], in this essay we also study other classes of groups for which the converse of Baer's Theorem is true.
\end{abstract}




\section{Introdução}

Seja $G$ um grupo arbitrário. Denotamos por $Z(G)$ e $G^{\prime}$, respectivamente, o centro de $G$ e o subgrupo derivado de $G$. Intuitivamente tanto o índice $[G: Z(G)]$ quanto a ordem de $G^{\prime}$ medem o quanto $G$ é abeliano, pois o fato de $G$ ser abeliano é equivalente à condição de que $[G: Z(G)]=1$ e também é equivalente à condição de que $G^{\prime}=1$. Sendo assim, é natural se perguntar qual é a relação entre $[G: Z(G)]$ e a ordem de $G^{\prime}$ no caso geral em que $G$ não é necessariamente abeliano. Neste sentido, um teorema clássico de Issai Schur [25, 10.1.4] nos garante que se $[G: Z(G)]$ é finito então $G^{\prime}$ é finito. A prova deste teorema é uma aplicação da teoria do homomorfismo transfer. Posteriormente alguns autores forneceram outras provas do Teorema de Schur, algumas das quais se baseavam em conceitos mais elementares como identidades de comutadores, sem fazer uso do transfer (veja, por exemplo, [19, Theorem 8.19] e [27]).

Reforçando a relação entre $[G: Z(G)]$ e $\left|G^{\prime}\right|$ James Wiegold mostrou em $[30]$ que se $[G: Z(G)]$ é finito então $\left|G^{\prime}\right| \leq f([G: Z(G)])$, onde a expressão explícita da função é $f(n)=n^{\frac{1}{2}\left(\log _{p} n-1\right)}$, sendo $p$ o menor primo divisor de $[G: Z(G)]$. Wiegold ainda forneceu exemplos mostrando que esta cota é a melhor possível. O Teorema de Schur, com a versão quantitativa de Wiegold, diz informalmente que se $Z(G)$ é grande então $G^{\prime}$ é pequeno. 
Infelizmente a recíproca do Teorema de Schur não vale em geral. Como mostram os exemplos dos p-grupos extra especiais infinitos, o fato de $G^{\prime}$ ser finito não implica que $[G: Z(G)]$ seja finito. Um $p$-grupo $P$ é dito extra especial se $P^{\prime}$ e $Z(P)$ coincidem e têm ordem $p$ e $P / Z(P)$ é um p-grupo abeliano elementar infinito (veja a seção 1.5).

Apesar de não ser possível mostrar em geral a recíproca do Teorema de Schur, para algumas classes de grupos é possível estabelecer este fato. Por exemplo, caso $G$ seja finitamente gerado, ou residualmente finito, ou caso exista um subgrupo abeliano de índice finito em $G$, então $G^{\prime}$ ser finito implica que $[G: Z(G)]$ é finito. Estas recíprocas parciais do Teorema de Schur despertaram o interesse de alguns autores nos últimos anos. Veja, por exemplo, os artigos $[\mathbf{1 7}],[\mathbf{2 4}],[\mathbf{2 3}],[\mathbf{1 4}]$ e suas referências.

Dado um grupo $G$, denotamos por $Z_{i}(G)$ e por $\gamma_{i+1}(G)$ os termos das séries centrais ascendente e descendente de $G$, respectivamente. Notamos que o conceito de grupo nilpotente generaliza de certa forma o de grupo abeliano. Intuitivamente tanto $\left[G: Z_{i}(G)\right]$ quanto a ordem de $\gamma_{i+1}(G)$ medem o quanto $G$ é nilpotente de classe $i$, pois o fato de $G$ ser nilpotente de classe $i$ é equivalente à condição de que $\left[G: Z_{i}(G)\right]=1$ e também é equivalente a $\gamma_{i+1}(G)=1$. Dessa forma, também é natural se perguntar qual é a relação entre $\left[G: Z_{i}(G)\right]$ e $\left|\gamma_{i+1}(G)\right|$ no caso geral em que $G$ não é necessariamente nilpotente de classe $i$. Respondendo esses questionamentos Reinhold Baer [2, §6 Theorem 4] generalizou o Teorema de Schur para os outros termos das séries centrais ascendente e descendente, mostrando que se $\left[G: Z_{i}(G)\right]$ é finito então $\gamma_{i+1}(G)$ é finito. Assim como Wiegold mostrou que a ordem de $G^{\prime}$ é limitada 
em função de $[G: Z(G)]$, também é possível mostrar que a ordem de $\gamma_{i+1}(G)$ é limitada em função de $\left[G: Z_{i}(G)\right]$.

Em [11] Philip Hall fornece, para cada inteiro $i \geq 1$, um exemplo de um p-grupo $G$ tal que $\gamma_{i+1}(G)$ é finito e $\left[G: Z_{i}(G)\right]$ é infinito, mostrando que a recíproca do Teorema de Baer não é válida em geral (veja as páginas 47-49 desta dissertação). A despeito disso, nesse mesmo artigo, P. Hall mostrou que se $\gamma_{i+1}(G)$ é finito então $\left[G: Z_{2 i}(G)\right]$ é finito, onde $i$ é um inteiro não negativo. Analisando mais atentamente a demonstração dada por P. Hall, é possível deduzir que se $\gamma_{i+1}(G)$ é finito de ordem $n$ então $\left[G: Z_{2 i}(G)\right] \leq n^{\log _{2} n+\left(\log _{2} n\right)^{2 i}}$ (veja [26, p. $118])$.

O Teorema de Hall mostra que um grupo finito-por-nilpotente é de fato nilpotente-por-finito, e pode ser visto como uma recíproca parcial do Teorema de Baer. Outras recíprocas parciais do Teorema de Baer podem ser obtidas impondo condições adicionais ao grupo $G$ como, por exemplo, a condição de que $\gamma_{i+1}(G) \cap Z_{i}(G)=1$, a condição de que $G$ seja residualmente finito $[20]$ ou ainda a condição de que $G$ seja finitamente gerado [16]. Recentemente foi publicado o artigo [14] dedicado a uma recíproca do Teorema de Baer para um grupo $G$ tal que $G / Z_{i}(G)$ é finitamente gerado.

Ian Macdonald [20, Corollary 2], enfraquecendo as hipóteses do Teorema de Hall, mostrou que se $G$ é um grupo tal que, para algum $i \geq 1,\left[\gamma_{i+1}(G): \gamma_{i+1}(G) \cap Z(G)\right]$ é finito então $\left[G: Z_{2 i}(G)\right]$ é finito. Recentemente Podoski e Szegedy [24] mostraram que se $\left[G^{\prime}: G^{\prime} \cap Z(G)\right]$ é finito e igual a $n$ então $\left[G: Z_{2}(G)\right] \leq n^{2 \log _{2} n}$, e que essa cota está próxima de ser a melhor possível. 
Gustavo Fernández-Alcober e Marta Morigi [7] generalizaram, para qualquer $i \geq 1$, o resultado de Podoski e Szegedy obtendo a seguinte versão mais forte do Teorema de Hall:

Teorema A. Sejam $G$ um grupo e um inteiro $i \geq 0$ tais que $\left[\gamma_{i+1}(G): \gamma_{i+1}(G) \cap Z_{i}(G)\right]$ é finito. Então $\left[G: Z_{2 i}(G)\right]$ é finito e limitado em função de $\left[\gamma_{i+1}(G): \gamma_{i+1}(G) \cap Z_{i}(G)\right]$.

Tendo em vista essa série de generalizações sucessivas do Teorema de Hall, surgem naturalmente as seguintes questões:

(a) Se a hipótese mais fraca de que $\left[\gamma_{i+1}(G): \gamma_{i+1}(G) \cap Z_{i+1}(G)\right]$ é finito vale, ainda é possível mostrar que $\left[G: Z_{2 i}(G)\right]$ é finito?

(b) Seja $G$ um grupo finitamente gerado. Em [16, Theorem 2.10] Hekster mostrou que se $\gamma_{i+1}(G)$ é finito então $\left[G: Z_{i}(G)\right]$ é finito. É possível mostrar que $\left[G: Z_{i}(G)\right]$ é finito sob a hipótese mais fraca do Teorema A de que $\left[\gamma_{i+1}(G): \gamma_{i+1}(G) \cap Z_{i}(G)\right]$ é finito? É possível mostrar pelo menos que $\left[G: Z_{j}(G)\right]$ é finito para algum $j<2 i$ ?

Em [7] os autores fornecem exemplos que dão resposta negativa a ambas as questões (veja as páginas 76-79 desta dissertação).

Outro resultado de [7] que merece ser destacado é o teorema a seguir, que é uma ferramenta usada na demonstração do Teorema A.

Teorema B. Seja $G$ um grupo em que $\left[\gamma_{s}(G): \gamma_{s}(G) \cap Z_{t}(G)\right]$ é finito, para s et inteiros. Então $\left[\gamma_{s+j}(G): \gamma_{s+j}(G) \cap Z_{t-j}(G)\right]$ é finito, para todo $0 \leq j \leq t$. Em particular, $\gamma_{s+t}(G)$ é finito.

Como caso particular do Teorema B, para $s=i+1$ e $t=i$, temos que se $\left[\gamma_{i+1}(G): \gamma_{i+1}(G) \cap Z_{i}(G)\right]$ é finito então $\gamma_{2 i+1}(G)$ é finito. Ressaltamos ainda que o Teorema B já havia sido apresentado por 
Macdonald [20, Corollary 1] com uma prova diferente da fornecida em $[7]$.

Seguindo M. Hall e Senior [10], dizemos que um grupo G é capable se for isomorfo a $H / Z(H)$, para algum grupo $H$. Em [13] P. Hall ressalta que a compreensão dos grupos capable é importante para a classificação dos p-grupos. Em [17] e [24] são provadas recíprocas do Teorema de Schur para grupos capable. Sucessivamente o conceito de grupo capable foi generalizado por Burns e Ellis em [4] da seguinte forma: dado um inteiro $i \geq 1$, dizemos que um grupo $G$ é $i$-capable se $G$ é isomorfo a $H / Z_{i}(H)$, para algum grupo $H$. Na Seção 3.2 mostramos que o Teorema A de Fernández-Alcober e Morigi é equivalente à recíproca do Teorema de Baer para grupos $i$-capable.

Este trabalho está dividido em 3 capítulos. O Capítulo 1 é dedicado ao tratamento dos resultados e definições mais básicos para a compreensão dos capítulos seguintes. No Capítulo 2 são apresentados os teoremas clássicos de Schur, Baer e Hall com suas versões quantitativas, bem como várias recíprocas parciais dos Teoremas de Schur e de Baer e ainda alguns contra exemplos. No Capítulo 3 detalhamos os resultados de [7] e discutimos a interpretação do Teorema A no contexto dos grupos $i$-capable. 


\section{CAPíTULO 1}

\section{Preliminares}

Usamos como principais referências os livros [25] de Derek Robinson e [18] de Martin Isaacs. Tentamos adotar uma notação padrão em Teoria de Grupos sempre que possível. Em geral, $G$ denota um grupo qualquer, não necessariamente finito. Usamos o símbolo 1 tanto para o elemento identidade quanto para o grupo que contém apenas a identidade. Quando $H$ é um subgrupo de um grupo $G$, escrevemos $H \leq G$. O índice de $H$ em $G$, denotado por $[G: H]$, é a cardinalidade do conjunto das classes laterais à esquerda (ou à direita) de $H$ em $G$. O símbolo $|X|$ denota a cardinalidade do conjunto $X$. As letras $m$ e $n$ em geral denotam inteiros positivos, sendo um primo normalmente denotado por $p$. Na maioria das vezes escrevemos $\alpha(x)$ para denotar a imagem de $x$ pela função $\alpha$, mas em algumas raras situações usamos $x^{\alpha}$ para a imagem de $x$ pela função $\alpha$.

Assumimos que o leitor tem familiaridade com os conceitos básicos da Teoria de Grupos. Alguns teoremas como o Teorema de Lagrange e os Teoremas de Isomorfismo também são assumidos como conhecidos.

Vamos iniciar demonstrando duas propriedades básicas dos índices de subgrupos que são muito usadas neste trabalho.

Proposição 1.0.1. Se $H$ e $K$ são subgrupos de um grupo $G$ então $[H: H \cap K]=|\{h K \mid h \in H\}|$. Em particular, se $H K$ é um subgrupo então $[H: H \cap K]=[H K: K]$. 
DemonstraÇÃo. Seja $\mathfrak{C}$ o conjunto das classes laterais à esquerda de $H \cap K$ em $H$. Vamos mostrar que $\varphi: h(H \cap K) \mapsto h K$ é uma bijeção de $\mathfrak{C}$ em $\{h K \mid h \in H\}$. Note que, $h_{1}(H \cap K)=h_{2}(H \cap K)$ se, e somente se, $h_{1}^{-1} h_{2} \in H \cap K \subseteq K$, que é equivalente a $h_{1} K=h_{2} K$. Isto mostra que $\varphi$ está bem definida e é injetiva. Além disso, $\varphi$ é claramente sobrejetiva. Isto conclui a demonstração.

Proposição 1.0.2. Seja $G$ um grupo. Se os subgrupos $H_{1}, \ldots, H_{n}$ todos têm índices finitos em $G$ então $H_{1} \cap \cdots \cap H_{n}$ também tem índice finito em $G$, e $\left[G: H_{1} \cap \cdots \cap H_{n}\right] \leq\left[G: H_{1}\right] \cdots\left[G: H_{n}\right]$.

Demonstração. Chamemos de $\mathfrak{C}$ o conjunto das classes laterais à esquerda de $H_{1} \cap \cdots \cap H_{n}$ em $G$ e, para $1 \leq i \leq n$, chamemos de $\mathfrak{C}_{i}$ o conjunto das classes laterais à esquerda de $H_{i}$ em $G$. Definimos $\varphi: \mathfrak{C} \longrightarrow \mathfrak{C}_{1} \times \cdots \times \mathfrak{C}_{n}$ tal que $\varphi\left(g\left(H_{1} \cap \cdots \cap H_{n}\right)\right)=\left(g H_{1}, \ldots, g H_{n}\right)$. Vamos mostrar que $\varphi$ está bem definida e é injetiva. Sejam $a$ e $b$ elementos arbitrários de $G$. Note que $a\left(H_{1} \cap \cdots \cap H_{n}\right)=b\left(H_{1} \cap \cdots \cap H_{n}\right)$ se, e somente se, $a^{-1} b \in H_{1} \cap \cdots \cap H_{n}$, o que é equivalente a $a^{-1} b \in H_{i}$, para todo $1 \leq i \leq n$. Isto, por sua vez, é equivalente à equação $\left(a H_{1}, \ldots, a H_{n}\right)=\left(b H_{1}, \ldots, b H_{n}\right)$. Isto mostra que $\varphi$ está bem definida e é injetiva, e o resultado segue.

\subsection{Subgrupos finitamente gerados e a condição maximal}

Seja $X$ um conjunto não vazio de um grupo $G$. O subgrupo gerado por $X$, denotado por $\langle X\rangle$, é o menor subgrupo de $G$ que contém $X$. Mostra-se que $\langle X\rangle=\left\{x_{1} \cdots x_{n} \mid n \geq 0, x_{i} \in X \cup X^{-1}\right\}$, onde denota$\operatorname{mos} X^{-1}=\left\{x^{-1} \mid x \in X\right\}$ e por convenção a palavra vazia é igual a identidade. 
Um grupo $G$ é dito finitamente gerado se existe um subconjunto finito $X \subseteq G$ tal que $G=\langle X\rangle$. Se $n$ é um inteiro positivo, $G$ é dito $n$-gerado se pode ser gerado por algum subconjunto $X \subseteq G$ com $n$ elementos, onde o conjunto $X$ é pensado ser não redundante, no sentido de que nenhum subconjunto próprio de $X$ pode gerar $G$.

Proposição 1.1.1. Se um grupo $G$ possui um subgrupo normal $N$ tal que $N$ é n-gerado e $G / N$ é m-gerado, então $G$ é $(n+m)$-gerado.

Demonstração. Seja $X \subset N$ tal que $N=\langle X\rangle$ e $|X|=n$. Seja $Y=\left\{g_{1}, \ldots, g_{m}\right\} \subseteq G$ tal que $G / N=\left\langle g_{1} N, \ldots, g_{m} N\right\rangle$. Dado qualquer $g \in G$, podemos escrever $g N=y_{1} \cdots y_{r} N$, com $y_{1}, \ldots, y_{r} \in Y \cup Y^{-1}$. Logo, $g=y_{1} \cdots y_{r} x, \operatorname{com} x \in N$. Observamos que $x=x_{1} \cdots x_{s}$, com $x_{1}, \ldots, x_{s} \in X \cup X^{-1}$. Segue que $g=y_{1} \cdots y_{r} x_{1} \cdots x_{s}$. Assim, $G=\langle X \cup Y\rangle$, sendo $|X \cup Y|=m+n$.

Um elemento $g$ de um grupo $G$ tem ordem finita $n$ se o grupo $\langle g\rangle$ tem ordem $n$. Se $\langle g\rangle$ é infinito, dizemos que $g$ tem ordem infinita. Um grupo no qual todos os elementos tem ordem finita é dito periódico. Um grupo no qual a ordem de todos os elementos é finita e limitada é um grupo de exponente finito. O expoente do grupo é o mínimo múltiplo comum entre as possíveis ordens de elementos do grupo. Ou seja, um grupo $G$ tem expoente finito $e$ se $g^{e}=1$, para todo $g \in G$.

Obviamente, todo grupo finito tem expoente finito, mas a soma direta de infinitas cópias de $\mathbb{Z} / 2 \mathbb{Z}$ é um exemplo de um grupo de expoente finito que não é finito. Todo grupo de expoente finito é periódico, mas $C_{2^{\infty}}=\left\langle a_{1}, a_{2}, \ldots \mid a_{1}^{2}=1, a_{i}^{2}=a_{i-1}, \forall i>1\right\rangle$ é um exemplo de um grupo periódico que não tem expoente finito. 
Estes exemplos mostram que, mesmo para grupos abelianos, o grupo ter expoente finito não implica que ele é finito. Mas, para grupos abelianos finitamente gerados, expoente finito implica em finitude, como mostra a proposição a seguir.

Lema 1.1.2. Seja $G$ um grupo abeliano m-gerado. Se, para algum inteiro e, $G^{e}=1$ então $|G| \leq e^{m}$.

DemonstraÇÃo. Sejam $g_{1}, \ldots, g_{m} \in G$ tais que $G=\left\langle g_{1}, \ldots, g_{m}\right\rangle$. Dado $g \in G$, como $G$ é abeliano podemos escrever $g=g_{1}^{\alpha_{1}} \cdots g_{m}^{\alpha_{m}}$, com $0 \leq \alpha_{i}<e$, pois $g_{i}^{e}=1$ para todo $1 \leq i \leq m$. Portanto, $|G| \leq e^{m}$.

Denotamos por $\lfloor x\rfloor$ a parte inteira do número real $x$.

ProposiçÃo 1.1.3. Seja $H$ um subgrupo próprio de um grupo $G$ tal $q u e[G: H]$ é finito e seja p é o menor primo divisor de $[G: H]$. Então existem um inteiro $1 \leq m \leq\left\lfloor\log _{p}[G: H]\right\rfloor$ e elementos $x_{1}, \ldots, x_{m} \in G$ tais que $G=\left\langle x_{1}, \ldots, x_{m}, H\right\rangle$.

Demonstração. Defina $H_{0}=H$ e, para $j \geq 1$, defina recursivamente $H_{j}=\left\langle x_{j}, H_{j-1}\right\rangle$, onde $x_{j}$ é escolhido arbitrariamente em $G \backslash H_{j-1}$, enquanto for $H_{j-1}<G$. Como $[G: H]$ é finito, temos que $H_{m}=G$, para algum $m \geq 1$. Temos, assim, a série de subgrupos $H=H_{0}<H_{1}<\cdots<H_{m}=G$. Note que $p \leq\left[H_{j}: H_{j-1}\right]$, para todo $1 \leq j \leq m$, pois $\left[H_{j}: H_{j-1}\right]>1$ e $\left[H_{j}: H_{j-1}\right]$ divide $[G: H]$. Assim,

$$
[G: H]=\left[H_{m}: H_{m-1}\right] \cdots\left[H_{1}: H_{0}\right] \geq p^{m} .
$$

Logo, $m \leq \log _{p}[G: H]$ e, como $m$ é inteiro, $m \leq\left\lfloor\log _{p}[G: H]\right\rfloor$. Finalmente, $H_{m}=\left\langle x_{m}, H_{m-1}\right\rangle=\cdots=\left\langle x_{1}, \ldots, x_{m}, H_{0}\right\rangle=\left\langle x_{1}, \ldots, x_{m}, H\right\rangle$. Isto conclui a demonstração. 
1.1. SUBGRUPOS FINITAMENTE GERADOS E A CONDIÇÃO MAXIMAL 5

Observe que, para todo primo $p$, temos $\log _{p}[G: H] \leq \log _{2}[G: H]$ então, na Proposição 1.1.3 também podemos obter $m \leq\left\lfloor\log _{2}[G: H]\right\rfloor$.

Seja $G$ um grupo finitamente gerado. Denotamos por $d(G)$ o número mínimo de elementos necessário para gerar $G$, onde por convenção $d(G)=0$ se $G=1$.

Corolário 1.1.4. Se $G$ é um grupo finito então $d(G) \leq\left\lfloor\log _{p}|G|\right\rfloor$, onde p é o menor primo divisor da ordem de $G$.

Segue da Proposição 1.1.3 com $H=1$.

Observe que $\log _{p}|G| \leq \log _{2}|G|$ então, do Corolário 1.1.4 concluímos que $d(G) \leq\left\lfloor\log _{2}|G|\right\rfloor$, qualquer que seja o grupo $G$. As vezes é mais conveniente usar essa desigualdade.

Dizemos que um grupo $G$ satisfaz a condição maximal se o conjunto dos subgrupos de $G$, parcialmente ordenado pela inclusão, não possui nenhuma cadeia ascendente infinita $H_{1}<H_{2}<\cdots$. Isto é, toda cadeia ascendente infinita de subgrupos de $G$ se torna estacionária a partir de algum ponto.

ProposiçÃo 1.1.5. Um grupo $G$ satisfaz a condição maximal se, e somente se, todos os seus subgrupos são finitamente gerados.

DemonstraçÃo. Assuma que $G$ satisfaz a condição maximal e suponha que exista um subgrupo $H \leq G$ que não é finitamente gerado. Escolha $h_{1} \in H$. Como $H$ não é finitamente gerado, temos que $\left\langle h_{1}\right\rangle \neq H$, de forma que podemos escolher $h_{2} \in H \backslash\left\langle h_{1}\right\rangle$. Continuando assim, obtemos uma cadeia ascendente infinita $\left\langle h_{1}\right\rangle<\left\langle h_{1}, h_{2}\right\rangle<\cdots$ de subgrupos de $G$. Isso contradiz a suposição de que $G$ satisfaz a condição maximal. 
Agora assuma que todos os subgrupos de $G$ são finitamente gerados e considere uma cadeia arbitrária $H_{1} \leq H_{2} \leq \cdots$ de subgrupos de $G$. Vamos mostrar que essa cadeia não é infinita. O conjunto $U=\bigcup_{i=1}^{\infty} H_{i}$ é um subgrupo de $G$. Como todo subgrupo de $G$ é finitamente gerado, existem $u_{1}, \ldots, u_{n} \in U$ tais que $U=\left\langle u_{1}, \ldots, u_{n}\right\rangle$. Note que existe um inteiro $r$ suficientemente grande tal que $u_{1}, \ldots, u_{n} \in H_{r}$. Assim, $H_{r}=U$ e a cadeia fica estacionária a partir de $H_{r}$. Portanto, $G$ satisfaz a condição maximal.

ProposiçÃo 1.1.6. Seja $G$ um grupo. Se $G$ possui um subgrupo normal $N$ tal que $N$ e $G / N$ satisfazem a condição maximal então $G$ satisfaz a condição maximal.

Demonstração. Pela Proposição 1.1.5, basta mostrar que todo subgrupo de $G$ é finitamente gerado. Seja $H$ um subgrupo de $G$. Notamos que, pela Proposição 1.1.5, $H \cap N$ é finitamente gerado, pois $N$ satisfaz a condição maximal. Analogamente, $H N / N$ é finitamente gerado, pois $G / N$ satisfaz a condição maximal. Como $H \cap N$ é normal em $H$, podemos considerar o quociente $H /(H \cap N)$, o qual é finitamente gerado, pois é isomorfo a $H N / N$. Portanto, segue da Proposição 1.1.1 que $H$ é finitamente gerado.

Proposição 1.1.7. Seja $G$ um grupo abeliano. Se $G$ é m-gerado então todo subgrupo de $G$ é m-gerado. Em particular, $G$ satisfaz a condição maximal.

DemonstraçÃo. Argumentamos por indução sobre $m$. Se $m=1$ então $G$ é cíclico e, portanto, todo subgrupo de $G$ é cíclico. Agora, seja $G=\left\langle g_{1}, \ldots, g_{m+1}\right\rangle$. Considere o subgrupo $N=\left\langle g_{1}, \ldots, g_{m}\right\rangle$. Por indução, todo subgrupo de $N$ é $m$-gerado e note que $G / N$ é cíclico. Se 
1.1. SUBGRUPOS FINITAMENTE GERADOS E A CONDIÇÃO MAXIMAL 7 $H$ é um subgrupo de $G$, então $H \cap N$ é $m$-gerado e $H /(H \cap N) \cong H N / N$, que é cíclico. Segue da Proposição 1.1.1 que $H$ é $(m+1)$-gerado.

Em geral nem todo subgrupo de um grupo finitamente gerado é finitamente gerado, como mostra o exemplo a seguir.

Considere o grupo $G=\left\langle a, b \mid b^{-1} a b=a^{2}\right\rangle$ e o subgrupo normal $H=\left\langle a, a^{b^{-1}}, a^{b^{-2}}, \ldots\right\rangle$. Veremos dentre outras coisas que $H$ não é finitamente gerado. Para verificar que $H$ é de fato normal em $G$ basta mostrar que $H^{a} \subseteq H$ e que $H^{b} \subseteq H$, pois $G=\langle a, b\rangle$. Claramente $H^{a} \subseteq H$, pois $a \in H$, e, como $\left(a^{b^{-i}}\right)^{b} \in H$, para todo $i \geq 0$, segue que $H^{b} \subseteq H$, pois $H$ é gerado pelos elementos da forma $a^{b^{-i}}$, com $i \geq 0$. Por comodidade definimos $x_{i}=a^{b^{-i}}$, para $i \geq 0$, de forma que $H=\left\langle x_{0}, x_{1}, x_{2} \ldots\right\rangle$. Observe que

$$
x_{i}=x_{i+1}^{2} \quad \text { para todo } i \geq 0
$$

pois $x_{i+1}^{2}=\left(a^{b^{-(i+1)}}\right)^{2}=\left(a^{2}\right)^{b^{-(i+1)}}=\left(a^{b}\right)^{b^{-(i+1)}}=a^{b^{-i}}=x_{i}$. Para ver que $H$ é abeliano basta mostrar que quaisquer $x_{i}, x_{j} \in\left\{x_{0}, x_{1}, x_{2}, \ldots\right\}$ comutam entre si. Isto segue da equação (1.1.1). De fato, podemos assumir sem perda de generalidade que $i<j$ e, assim, aplicando (1.1.1) repetidas vezes, temos que $x_{i}=x_{i+1}^{2}=\cdots=x_{j}^{2^{j-i}}$. Logo, $x_{i} \in\left\langle x_{j}\right\rangle \mathrm{e}$ em particular $x_{i}$ comuta com $x_{j}$. De (1.1.1) também segue que

$$
\left\langle x_{0}\right\rangle \leq\left\langle x_{1}\right\rangle \leq \cdots \leq\left\langle x_{i}\right\rangle \leq\left\langle x_{i+1}\right\rangle \leq \cdots
$$

Agora, como $H$ é abeliano, todo elemento $h \in H$ se escreve como $h=x_{i_{1}}^{n_{1}} \cdots x_{i_{s}}^{n_{s}}$, com $x_{i_{1}}, \ldots, x_{i_{s}} \in\left\{x_{0}, x_{1}, x_{2}, \ldots\right\}$ e $n_{1}, \ldots, n_{s}$ inteiros. Escolhendo $i=\max \left(i_{1}, \ldots, i_{s}\right)$, temos que $x_{i_{1}}, \ldots, x_{i_{s}} \in\left\langle x_{i}\right\rangle$ e portanto 
1.1. SUBGRUPOS FINITAMENTE GERADOS E A CONDIÇÃO MAXIMAL 8 $h \in\left\langle x_{i}\right\rangle$. Isto mostra que $H \subseteq \bigcup_{n=1}^{\infty}\left\langle x_{n}\right\rangle$. Assim,

$$
H=\bigcup_{n=1}^{\infty}\left\langle x_{n}\right\rangle \text {. }
$$

Com isso, podemos mostrar que $H$ não é finitamente gerado. De fato, se $Y \subseteq H$ é um conjunto finito qualquer, então $Y \subseteq\left\langle x_{k}\right\rangle$, para algum inteiro positivo $k$ suficientemente grande. Logo, $\langle Y\rangle \subseteq\left\langle x_{k}\right\rangle \neq H$. Ou seja, $H$ não é finitamente gerado.

Este exemplo mostra que um subgrupo $H$ de um grupo finitamente gerado $G$ pode não ser finitamente gerado. De fato, o exemplo mostra que, mesmo subgrupos normais abelianos do grupo $G$, podem não ser finitamente gerados. Não obstante, dado um grupo finitamente gerado, os subgrupos de índice finito são sempre finitamente gerados. Este é o conteúdo do teorema a seguir.

Teorema 1.1.8 (Schreier). Sejam G um grupo finitamente gerado. Se $H$ é um subgrupo de $G$ com indice finito então $H$ é finitamente gerado. Mais precisamente, se $G$ é m-gerado e $[G: H]=n$ então $H$ pode ser gerado por mn elementos.

DemonstraÇÃo. Seja $G=\langle X\rangle, \operatorname{com}|X|=m$, e $T$ um transversal à direita para $H$ em $G$. Assuma que $1 \in T$ e note que $|T|=n$. Para todo $g \in G$ e todo $t \in T$, existe um único $\tau(g) \in T$ tal que $H t g=H \tau(g)$. Defina $h(t, g)=t g \tau(g)^{-1}$ e note que $h(t, g) \in H$ e que

$$
t g=h(t, g) \tau(g)
$$

Dado $h \in H$, temos $h=g_{1} \cdots g_{s}$, com $g_{1}, \ldots, g_{s} \in X \cup X^{-1}$. Usando a fórmula (1.1.2), temos que $1 g_{1}=h\left(1, g_{1}\right) \tau\left(g_{1}\right)$. De forma que o elemento $h$ se reescreve como $h=1 g_{1} g_{2} \cdots g_{s}=h\left(1, g_{1}\right) \tau\left(g_{1}\right) g_{2} \cdots g_{s}$. 
Aplicando (1.1.2) novamente, vemos que $\tau\left(g_{1}\right) g_{2}=h\left(\tau\left(g_{1}\right), g_{2}\right) \tau\left(g_{2}\right)$. Repetindo esse processo, obtemos

$$
h=h\left(1, g_{1}\right) h\left(\tau\left(g_{2}\right), g_{2}\right) \cdots h\left(\tau\left(g_{s-1}\right), g_{s}\right) \tau\left(g_{s}\right)
$$

Assim, $H \tau\left(g_{s}\right)=H h=H$ e tem-se necessariamente $\tau\left(g_{s}\right)=1$, pois $T$ é um transversal. Logo, da equação (1.1.3) segue que $H$ é gerado pelo conjunto finito $Y=\left\{h(t, g) \mid t \in T, g \in X \cup X^{-1}\right\}$, que tem no máximo $2 m n$ elementos.

Agora veremos que, na verdade, precisamos apenas de metade dos elementos de $Y$ para gerar $H$. Para todo $g \in G$ e todo $t \in T$, temos que $H t g^{-1}=H \tau\left(g^{-1}\right)$, ou seja, $H \tau\left(g^{-1}\right) g=H t$ e então temos que $h\left(\tau\left(g^{-1}\right), g\right)=\tau\left(g^{-1}\right) g t^{-1}$. Por outro lado, $h\left(t, g^{-1}\right)=t g^{-1} \tau\left(g^{-1}\right)^{-1} \mathrm{e}$, portanto,

$$
h\left(t, g^{-1}\right)=h\left(\tau\left(g^{-1}\right), g\right)^{-1}
$$

Concluímos que $Y \subseteq\langle h(t, g) \mid t \in T, g \in X\rangle$ e, como $H=\langle Y\rangle$, temos que $H=\langle h(t, g) \mid t \in T, g \in X\rangle$. Observe que esse conjunto gerador tem no máximo mn elementos. Isto conclui a demonstração.

Notamos que a cota para o número de geradores de $H$ no Teorema 1.1.8 ainda pode ser melhorada, como mostra o teorema a seguir.

TeOrema 1.1.9. [25, 6.1.8(ii)] Sejam $G$ um grupo m-gerado e $H$ um subgrupo tal que $[G: H]=n$. Então $H$ pode ser gerado por $m n-n+1$ elementos.

\subsection{Sobre comutadores e centralizadores}

Dados elementos $a$ e $b$ de um grupo $G$, o comutador de $a$ e $b$ é o elemento $[a, b]=a^{-1} b^{-1} a b$. A identidade $a b=b a[a, b]$ justifica o nome 
comutador para o fator que aparece ao se comutar dois elementos. O conjugado de $a$ por $b$ é o elemento $a^{b}=b^{-1} a b$. Dados um inteiro $n \geq 2$ e elementos $x_{1}, \ldots, x_{n} \in G$, o comutador simples de peso $n$ é definido recursivamente como $\left[x_{1}, \ldots, x_{n}\right]=\left[\left[x_{1}, \ldots, x_{n-1}\right], x_{n}\right]$. Por convenção $\left[x_{1}\right]=x_{1}$.

Existem algumas relações entre comutadores e conjugados. Por exemplo, $[a, b]=a^{-1} a^{b}$ e também $[a, b]^{x}=\left[a^{x}, b^{x}\right]$, valem para quaisquer elementos $a, b, x \in G$. Esta última é consequência de a aplicação $g \mapsto g^{x}$ ser um automorfismo de $G$.

As identidades a seguir, que são de fácil verificação, são usadas diversas vezes neste trabalho. Dados $x, y, g \in G$, temos que

$$
\begin{aligned}
& {[x y, g]=[x, g]^{y}[y, g],} \\
& {[g, x y]=[g, y][g, x]^{y} .}
\end{aligned}
$$

Tomando por caso base as identidades (1.2.1) e (1.2.2), mostra-se por indução que, dados $n \geq 2$ e $x_{1}, \ldots, x_{n}, g \in G$,

$$
\begin{gathered}
{\left[x_{1} \cdots x_{n}, g\right]=\left[x_{1}, g\right]^{x_{2} \cdots x_{n}} \cdots\left[x_{n-1}, g\right]^{x_{n}}\left[x_{n}, g\right],} \\
{\left[g, x_{1} \cdots x_{n}\right]=\left[g, x_{n}\right]\left[g, x_{n-1}\right]^{x_{1}} \cdots\left[g, x_{1}\right]^{x_{n-1} \cdots x_{1}} .}
\end{gathered}
$$

Das fórmulas (1.2.1) e (1.2.2) e, usando o fato de $a^{b}=a[a, b]$, temos:

$$
\begin{aligned}
& {[x y, g]=[x, g][x, g, y][y, g],} \\
& {[g, x y]=[g, y][g, x][g, x, y] .}
\end{aligned}
$$


Destacamos, ainda, outras duas fórmulas que usaremos:

$$
\begin{aligned}
& {\left[x, y^{-1}\right]=[y, x]^{y^{-1}}=\left([x, y]^{-1}\right)^{y^{-1}}=\left([x, y]^{y^{-1}}\right)^{-1}} \\
& {\left[x^{-1}, y\right]=[y, x]^{x^{-1}}=\left([x, y]^{-1}\right)^{x^{-1}}=\left([x, y]^{x^{-1}}\right)^{-1} .}
\end{aligned}
$$

Dados subconjuntos não vazios $X$ e $Y$ de um grupo $G$, definimos o subgrupo comutador de $X$ e $Y$ como $[X, Y]=\langle[x, y] \mid x \in X, y \in Y\rangle$.

Dados um subconjunto não vazio $X$ de um grupo $G$ e um elemento $g \in G$, o conjugado de $X$ por $g$ é o conjunto $X^{g}=\left\{x^{g} \mid x \in X\right\}$. O normalizador de $X$ em $G$ é definido como $N_{G}(X)=\left\{g \in G \mid X^{g}=X\right\}$. Se $H \leq G$ então $N_{G}(H)$ é o maior subgrupo de $G$ no qual $H$ é normal. A proposição a seguir dá uma caracterização do normalizador usando comutadores.

Proposição 1.2.1. Sejam $H$ e $K$ subgrupos de um grupo $G$. Então $K \leq N_{G}(H)$ se, e somente se, $[H, K] \leq H$.

Demonstração. Por um lado, se $K \leq N_{G}(H)$, dados $h \in H$ e $k \in K,[h, k]=h^{-1} h^{k} \in H$ e, portanto, $[H, K] \leq H$. Por outro lado, se $[H, K] \leq H$ então, para todo $h \in H$ e todo $k \in K$, temos que $h^{k}=h[h, k] \in H$ e, assim, $K \leq N_{G}(H)$.

Em particular, se $H$ é um subgrupo normal de um grupo $G$, segue da Proposição 1.2 .1 que $[K, H] \leq H$, para todo $K \leq G$.

Seja $X$ um subconjunto não vazio de um grupo $G$. O subconjunto $C_{G}(X)=\left\{g \in G \mid x^{g}=x, \forall x \in X\right\}$ é o centralizador de $X$ em $G$. Não é difícil verificar que $C_{G}(X)$ é de fato um subgrupo de $G$.

O centro de $G$ é o subgrupo $Z(G)=\{z \in G \mid z g=g z, \forall g \in G\}$. Observe que $Z(G)=C_{G}(G)$. 
Sejam $H$ um subgrupo de um grupo $G$ e $X \subseteq G$ não vazio. Defini$\operatorname{mos} C_{H}(X)=H \cap C_{G}(X)=\left\{h \in H \mid x^{h}=x, \forall x \in X\right\}$ e chamamos $C_{H}(X)$ de o centralizador de $X$ em $H$.

Proposição 1.2.2. Sejam $N$ e $H$ subgrupos de um grupo G. Se $N$ normal em $G$ então $C_{H}(N)$ é normal em $H$ e se, além disso, $N$ é finito então $C_{H}(N)$ tem indice finito em $H$.

Demonstração. Consideremos o homomorfismo $\varphi: H \rightarrow \operatorname{Aut}(N)$ que associa a cada elemento $h \in H$ o automorfismo $\sigma_{h}: x \mapsto h^{-1} x h$. Observe que $C_{H}(N)$ é exatamente o $\operatorname{Ker}(\varphi)=\left\{h \in H \mid \sigma_{h}=I d\right\}$. Logo, $C_{H}(N)$ é normal em $H$. Note que o grupo $\operatorname{Sym}(N)$ das bijeções de $N$ é finito pois $N$ é finito e, consequentemente, $\operatorname{Aut}(N)$ é finito pois é subgrupo de $\operatorname{Sym}(N)$. Pelo Teorema do Isomorfismo, temos que $H / C_{H}(N)=H / \operatorname{Ker}(\varphi)$ é isomorfo a um subgrupo do grupo finito $\operatorname{Aut}(N)$ e, portanto, $H / C_{H}(N)$ é finito.

Dado um subgrupo $K$ de um grupo $G$, é fácil ver que a aplicação

$$
\begin{aligned}
\varphi: N_{G}(K) & \longrightarrow \operatorname{Aut}(K) \\
x & \longmapsto\left(k \mapsto k^{x}\right)
\end{aligned}
$$

é um homomorfismo. Portanto, pelo Teorema do Isomorfismo, temos a seguinte proposição.

Proposição 1.2.3. Seja $K$ um subgrupo de um grupo $G$. Então $C_{G}(K)$ é normal em $N_{G}(K)$ e $N_{G}(K) / C_{G}(K)$ é isomorfo a um subgrupo de $\operatorname{Aut}(K)$.

Um subconjunto $X$ de um grupo $G$ é dito um subconjunto normal de $G$ se $X^{g}=X$, para todo $g \in G$. 
Lema 1.2.4. Seja $G$ um grupo. Se $X$ é um subconjunto normal de $G$ então $\langle X\rangle$ é normal em $G$.

DemonstraÇÃo. Dado $h \in\langle X\rangle$, podemos escrever $h=x_{1}^{\alpha_{1}} \cdots x_{k}^{\alpha_{k}}$, com $x_{1}, \ldots, x_{k} \in X$ e $\alpha_{1}, \ldots, \alpha_{k} \in\{-1,1\}$. Como, para todo elemento $g \in G$, a conjugação por $g$ é um homomorfismo, temos que $h^{g}=\left(x_{1}^{g}\right)^{\alpha_{1}} \cdots\left(x_{k}^{g}\right)^{\alpha_{k}}$. Logo, $h^{g} \in\langle X\rangle$, pois $x_{i}^{g} \in X$, para todo $i=1, \ldots, k$. Portanto $\langle X\rangle$ é normal em $G$.

Proposição 1.2.5. Sejam $M$ e $N$ subgrupos normais de um grupo G. Então $[M, N]$ é normal em $G$.

Demonstração. Lembre-se que $[M, N]$ é gerado pelo conjunto $X=\{[m, n] \mid m \in M, n \in N\}$. Pelo Lema 1.2.4, basta ver que o conjunto $X$ é um subconjunto normal em $G$. Ora, dado $g \in G$, $[m, n]^{g}=\left[m^{g}, n^{g}\right]$. Então $[m, n]^{g} \in X$, pois, sendo $M$ e $N$ normais, $m^{g} \in M$ e $n^{g} \in N$. Portanto, $X$ é normal em $G$, e o resultado segue.

Dados subconjuntos não vazios $X_{1}, X_{2}, \ldots$ de um grupo $G$, definimos recursivamente os subgrupos $\left[X_{1}\right]=\left\langle X_{1}\right\rangle$ e, para $n \geq 2$, $\left[X_{1}, \ldots, X_{n}\right]=\left[\left[X_{1}, \ldots, X_{n-1}\right], X_{n}\right]$.

A seguir estabelecemos vários resultados básicos sobre subgrupos comutadores.

Proposição 1.2.6. Sejam $A, B$ e $C$ subgrupos normais de um grupo G. Então $[A B, C]=[A, C][B, C]$.

DemonstraÇÃo. Dados $a \in A, b \in B$ e $c \in C$, pela identidade (1.2.1), temos $[a b, c]=[a, c]^{b}[b, c] \in[A, C][B, C]$, pois $[A, C]$ é normal em $G$ pela Proposição 1.2.5. $\operatorname{Logo}[A B, C] \leq[A, C][B, C]$. Por outro lado, $[A, C] \leq[A B, C]$ e $[B, C] \leq[A B, C]$. Logo $[A, C][B, C] \leq$ $[A B, C]$, e o resultado segue. 
Por indução sobre $n$ mostra-se facilmente o seguinte corolário.

Corolário 1.2.7. Sejam $A_{1}, \ldots, A_{n}$ e $C$ subgrupos normais de um grupo G. Então $\left[A_{1} \cdots A_{n}, C\right]=\left[A_{1}, C\right] \cdots\left[A_{n}, C\right]$.

Proposição 1.2.8. Sejam $A, B, H$ e $N$ subgrupos de um grupo $G$, sendo $N$ normal. Se $[A, H] \leq N$ e $[B, H] \leq N$ então $[A B, H] \leq N$.

Demonstração. Lembramos que os geradores de $[A B, H]$ são da forma $[a b, h]$, com $h \in H, a \in A$ e $b \in B$. Segue da identidade (1.2.1) que $[a b, h]=[a, h]^{b}[b, h]$. Por hipótese, $[a, h] \in N,[b, h] \in N$ e então $[a, h]^{b} \in N$ pois $N$ é normal em $G$. Assim $[a b, h] \in N$ e, portanto, os geradores de $[H, A B]$ estão contidos em $N$. Isto conclui a demonstração.

A partir da Proposição 1.2.8 podemos obter o resultado abaixo por indução sobre $n$.

Corolário 1.2.9. Sejam $A_{1}, \ldots, A_{n}, H$ e $N$ subgrupos de um grupo $G$, sendo $N$ normal. Se $\left[A_{i}, H\right] \leq N$, para todo $1 \leq i \leq n$, então $\left[A_{1} \cdots A_{n}, H\right] \leq N$.

\subsection{As séries centrais ascendente e descendente}

A série central descendente de um grupo $G$ é definida recursivamente: $\gamma_{1}(G)=G$ e, para $n \geq 2$, definimos

$$
\gamma_{n}(G)=\left[\gamma_{n-1}(G), G\right]
$$

Lembramos que os subgrupos $\gamma_{n}(G)$ são normais em $G$. De fato são invariantes por qualquer endomorfismo de $G$. Chamamos o subgrupo $\gamma_{2}(G)=[G, G]$ de subgrupo derivado de $G$ e o denotamos por $G^{\prime}$. 
Proposição 1.3.1. Seja $G$ um grupo. Então $\gamma_{n}(G) / \gamma_{n+1}(G)$ é abeliano, para todo $n \geq 1$.

DemonstraÇÃo. Se $a, b \in G$ então $\left[a G^{\prime}, b G^{\prime}\right]=[a, b] G^{\prime}=G^{\prime}$, ou seja, $a G^{\prime}$ e $b G^{\prime}$ comutam. Isto mostra que $G / G^{\prime}$ é abeliano. Agora, se $n \geq 2$, temos que $\gamma_{n}(G)^{\prime}=\left[\gamma_{n}(G), \gamma_{n}(G)\right] \leq\left[\gamma_{n}(G), G\right]=\gamma_{n+1}(G)$. Logo, $\gamma_{n}(G) / \gamma_{n+1}(G)$ é isomorfo a $\left(\gamma_{n}(G) / \gamma_{n}(G)^{\prime}\right) /\left(\gamma_{n+1}(G) / \gamma_{n}(G)^{\prime}\right)$, que é abeliano pois $\gamma_{n}(G) / \gamma_{n}(G)^{\prime}$ é abeliano. Portanto $\gamma_{n}(G) / \gamma_{n+1}(G)$ é abeliano, como queríamos.

Sejam $H, K$ e $N$ subgrupos de um grupo $G$, sendo $N$ normal em G. Em $G / N$ o subgrupo $[H N / N, K N / N]$ é gerado pelas classes do tipo $[h N, k N]$, com $h \in H$ e $k \in K$. Por outro lado, $[H, K]$ é gerado pelos comutadores do tipo $[h, k]$, com $h \in H$ e $k \in K$, então a imagem $[H, K] N / N$ de $[H, K]$ pelo homomorfismo canônico de $G$ em $G / N$ é gerado pelos elementos do tipo $[h, k] N$, com $h \in H$ e $k \in K$. Como $[h N, k N]=[h, k] N$, para todo $h \in H$ e todo $k \in K$, segue que

$$
[H N / N, K N / N]=[H, K] N / N
$$

Proposição 1.3.2. Seja $N$ um subgrupo normal de um grupo $G$. Então $\gamma_{i}(G / N)=\gamma_{i}(G) N / N$, para todo $i \geq 1$.

Demonstração. Argumentamos por indução sobre $i$. Quando $i=1$ o resultado é trivial. Supondo que o resultado vale para $i$, temos que $\gamma_{i+1}(G / N)=\left[\gamma_{i}(G / N), G / N\right]=\left[\gamma_{i}(G) N / N, G / N\right]$. Da identidade (1.3.1) segue que $\left[\gamma_{i}(G) N / N, G / N\right]=\left[\gamma_{i}(G), G\right] N / N=\gamma_{i+1}(G) N / N$. Isto conclui a indução e o resultado segue. 
A série central ascendente de um grupo $G$ é definida recursivamente: $Z_{0}(G)=1$ e, para $n \geq 1, Z_{n}(G)$ é o subgrupo de $G$ tal que

$$
\frac{Z_{n}(G)}{Z_{n-1}(G)}=Z\left(\frac{G}{Z_{n-1}(G)}\right) \text {. }
$$

Usando indução não é difícil verificar que, para todo $n \geq 0$,

$$
Z_{n}(G)=\left\{x \in G \mid\left[x, g_{1}, \ldots, g_{n}\right]=1, \forall g_{1}, \ldots, g_{n} \in G\right\}
$$

De fato, o caso $n=0$ é trivial e, supondo que (1.3.2) vale para $n$, mostramos que (1.3.2) vale para $n+1$. Pela definição de $Z_{n+1}(G)$,

$$
\begin{aligned}
Z_{n+1}(G) & =\left\{x \in G \mid x Z_{n}(G) \in Z\left(G / Z_{n}(G)\right)\right\} \\
& =\left\{x \in G \mid\left[x, g_{1}\right] \in Z_{n}(G), \forall g_{1} \in G\right\} \\
& =\left\{x \in G \mid\left[x, g_{1}, \ldots, g_{n+1}\right]=1, \forall g_{i} \in G\right\}
\end{aligned}
$$

onde a última igualdade é consequência da hipótese de indução. Isto conclui a indução.

Como é usual, dados subgrupos $A$ e $B$ de um grupo $G$, vamos escrever: $\left[A,,_{0} B\right]=A$ e, para $n \geq 1,\left[A,_{n} B\right]=\left[\left[A_{,_{n-1}} B\right], B\right] . \mathrm{O}$ resultado a seguir é consequência direta da identidade (1.3.2).

Proposição 1.3.3. Sejam H um subgrupo de um grupo $G$ e $n \geq 0$. $O$ comutador $\left[H,_{n} G\right]=1$ se, e somente se, $H \leq Z_{n}(G)$.

Proposição 1.3.4 (Hirsch). Seja $N$ um subgrupo normal de um grupo $G$ tal que $N \cap Z(G)=1$. Então $N \cap Z_{n}(G)=1$, para todo $n \geq 1$.

Demonstração. Usamos indução sobre $n$. O caso $n=1$ é dado por hipótese. Assuma, por indução, que o resultado vale para $n$. 
Dado $x \in N \cap Z_{n+1}(G)$, temos que $[x, G] \leq N$, pois $N$ é normal, e $\left[x, Z_{n+1}(G)\right] \leq Z_{n}(G)$. Assim, $[x, G] \leq N \cap Z_{n}(G)=1$, pela hipótese de indução. Então $x \in N \cap Z(G)=1$. Isto mostra que $N \cap Z_{n+1}(G)=1$ e termina a indução.

A fórmula a seguir é a Identidade de Hall-Witt, que vale para quaisquer elementos $x, y$ e $z$ de um grupo $G$.

$$
\left[x, y^{-1}, z\right]^{y}\left[y, z^{-1}, x\right]^{z}\left[z, x^{-1}, y\right]^{x}=1
$$

Para verificá-la basta expandir os comutadores. A Identidade de Hall-Witt é uma ferramenta essencial para provar o próximo resultado.

Lema 1.3.5 (dos Três Subgrupos). Sejam H,K e L subgrupos de um grupo $G$. Se $[K, L, H]$ e $[L, H, K]$ estão contidos em um subgrupo normal $N$ de $G$, então $[H, K, L]$ também está contido em $N$. Em particular, $[H, K, L] \leq[K, L, H][L, H, K]$.

Demonstração. Dados $x \in H, y \in K, z \in L$, da Identidade de Hall-Witt 1.3.3 aplicada respectivamente ao trio $x, y^{-1}, z$ e ao trio $y, x^{-1}, z$ segue que

$$
\begin{aligned}
& {[x, y, z]=\left(\left(\left[y^{-1}, z^{-1}, x\right]^{z}\left[z, x^{-1}, y^{-1}\right]^{x}\right)^{-1}\right)^{y}} \\
& {[y, x, z]=\left(\left(\left[x^{-1}, z^{-1}, y\right]^{z}\left[z, y^{-1}, x^{-1}\right]^{y}\right)^{-1}\right)^{x}}
\end{aligned}
$$

Como $[K, L, H]=[L, K, H]$ e $[L, H, K]=[H, L, K]$ estão contidos em $N$, segue que os quatro elementos $\left[y^{-1}, z^{-1}, x\right],\left[z, x^{-1}, y^{-1}\right],\left[x^{-1}, z^{-1}, y\right]$ e $\left[z, y^{-1}, x^{-1}\right]$ pertencem a $N$. Sendo $N$ normal concluímos que

$$
[x, y, z] \text { e }[y, x, z] \in N
$$


Os geradores de $[H, K, L]$ são da forma $\left[c_{1} \cdots c_{n}, z\right]$, com $z \in L$ e $c_{1}, \ldots, c_{n} \in\{[x, y],[y, x] \mid x \in H, y \in K\}$. Usando a fórmula (1.2.3), temos que $\left[c_{1} \cdots c_{n}, z\right]=\left[c_{1}, z\right]^{c_{2} \cdots c_{n}} \cdots\left[c_{n-1}, z\right]^{c_{n}}\left[c_{n}, z\right]$. As inclusões em (1.3.4) mostram que, para cada $1 \leq i \leq n$, o comutador $\left[c_{i}, z\right] \in N$ e, assim, $\left[c_{1} \cdots c_{n}, z\right] \in N$, pois $N$ é normal. Isto mostra que os geradores de $[H, K, L]$ pertencem à $N$ e, portanto, $[H, K, L]$ está contido em $N$.

Para verificar a segunda parte basta notar que, sendo $H, K$ e $L$ normais em $G$, temos que $[K, L, H][L, H, K]$ é normal em $G$.

O Lema dos Três Subgrupos é uma ferramenta extremamente útil e ajuda a simplificar certas demonstrações que envolvem muitas fórmulas com comutadores.

Proposição 1.3.6. Sejam um grupo $G$ e inteiros $1 \leq i \leq j$. Então $\left[\gamma_{i}(G), Z_{j}(G)\right]=Z_{j-i}(G)$. Em particular, $Z_{i}(G)$ centraliza $\gamma_{i}(G)$.

Demonstração. Vamos argumentar por indução sobre $i$. Primeiro, $\left[G, Z_{j}(G)\right] \leq Z_{j-1}(G)$. Agora, pelo Lema dos Três Subgrupos, $\left[\gamma_{i+1}(G), Z_{j}(G)\right]=\left[\gamma_{i}(G), G, Z_{j}(G)\right] \leq\left[\gamma_{i}(G), Z_{j}(G), G\right]\left[G, Z_{j}(G), \gamma_{i}(G)\right]$. Este, por indução, está contido em $\left[Z_{j-i}(G), G\right]\left[Z_{j-1}(G), \gamma_{i}(G)\right]$, que, por sua vez, está contido em $Z_{j-i-1}(G)$, novamente pela hipótese de indução. Isto conclui a demonstração.

ProposiçÃo 1.3.7. Sejam $G$ um grupo e $i \geq 1$. Então, dados um inteiro $1 \leq n \leq i$ e elementos $z \in Z_{i-1}(G)$ e $g_{1}, \ldots, g_{i} \in G$, temos

$$
\left[g_{1}, \ldots, g_{n-1}, z g_{n}, g_{n+1}, \ldots, g_{i}\right]=\left[g_{1}, \ldots, g_{i}\right]
$$

Demonstração. Usamos indução sobre $i$. O caso $i=1$ é trivial pois $Z_{0}(G)=1$. Assumindo por indução que o resultado vale para todo inteiro menor que $i$, vamos mostrar que o resultado vale para $i$. Pela 
identidade (1.2.2), temos que

$$
\left[g_{1}, \ldots, g_{n-1}, z g_{n}\right]=\left[g_{1}, \ldots, g_{n}\right]\left[g_{1}, \ldots, g_{n-1}, z\right]^{g_{n}}
$$

Ponha $x=\left[g_{1}, \ldots, g_{n}\right]$ e $u=\left[g_{1}, \ldots, g_{n-1}, z\right]^{g_{n}}$. Pela Proposição 1.3.6, $\left[g_{1}, \ldots, g_{n-1}, z\right] \in Z_{i-n}(G)$ e, assim, $u \in Z_{i-n}(G)$. Como $Z_{i-n}(G)$ é normal, temos que $x u=v x$, para algum $v \in Z_{i-n}(G)$. Logo, da equação (1.3.5), temos que $\left[g_{1}, \ldots, g_{n-1}, z g_{n}\right]=x u=v x$. Portanto,

$$
\left[g_{1}, \ldots, g_{n-1}, z g_{n}, g_{n+1}, \ldots, g_{i}\right]=\left[v x, g_{n+1}, \ldots, g_{i}\right]=\left[x, g_{n+1}, \ldots, g_{i}\right]
$$

pela hipótese de indução, pois $v \in Z_{i-n}(G)$ e $x, g_{n+1}, \ldots, g_{i}$ são $i-n+1$ elementos de $G$. Isto conclui a indução.

Proposição 1.3.8. Sejam $G$ um grupo e $i, j$ inteiros não negativos. Então $Z_{i}\left(G / Z_{j}(G)\right)=Z_{i+j}(G) / Z_{j}(G)$.

DemonstraçÃo. Pela identidade (1.3.2), $x Z_{j}(G) \in Z_{i}\left(G / Z_{j}(G)\right)$ se, e somente se, $\left[x Z_{j}(G), g_{1} Z_{j}(G), \ldots, g_{i} Z_{j}(G)\right]=Z_{j}(G)$, quaisquer que sejam $g_{1} Z_{j}(G), \ldots, g_{i} Z_{j}(G) \in G / Z_{j}(G)$. Logo, o elemento $x Z_{j}(G)$ pertence a $Z_{i}\left(G / Z_{j}(G)\right)$ se, e somente se, $\left[x, g_{1}, \ldots, g_{i}\right] \in Z_{j}(G)$, quaisquer que sejam $g_{1}, \ldots, g_{i} \in G$. Novamente pela identidade (1.3.2), isso acontece se, e somente se, $\left[\left[x, g_{1}, \ldots, g_{i}\right], g_{i+1}, \ldots, g_{i+j}\right]=1$, para quaisquer $g_{1}, \ldots, g_{i+j} \in G$. Ou seja, $x Z_{j}(G) \in Z_{i}\left(G / Z_{j}(G)\right)$ se, e somente se, $x \in Z_{i+j}(G)$, que é equivalente a $x Z_{j}(G) \in Z_{i+j}(G) / Z_{j}(G)$. Mostramos que um elemento arbitrário de $G / Z_{j}(G)$ pertence a $Z_{i}\left(G / Z_{j}(G)\right)$ se, e somente se, pertence a $Z_{i+j}(G) / Z_{j}(G)$. Isto conclui a demonstração.

Concluímos esta seção provando alguns resultados sobre a geração dos termos da série central descendente. 
ProposiçÃo 1.3.9. Seja $G$ um grupo e $n \geq 1$. Então

$$
\gamma_{n}(G)=\left\langle\left[g_{1}, \ldots, g_{n}\right] \mid g_{1}, \ldots, g_{n} \in G\right\rangle
$$

DemonstraçÃo. Argumentamos por indução sobre $n$. O caso $n=$ 1 é trivial. Assuma por indução que $\gamma_{n}(G)$ é gerado pelo conjunto $X=\left\{\left[g_{1}, \ldots, g_{n}\right] \mid g_{1}, \ldots, g_{n} \in G\right\}$. Por definição,

$$
\gamma_{n+1}(G)=\left\langle[h, g] \mid h \in \gamma_{n}(G), g \in G\right\rangle
$$

Então, pela hipótese de indução, um elemento gerador de $\gamma_{n+1}(G)$ pode ser escrito como $\left[c_{1} \cdots c_{k}, g\right]$, com $g \in G$ e $c_{1}, \ldots, c_{k} \in X \cup X^{-1}$. Pela fórmula (1.2.3), temos que

$$
\left[c_{1} \cdots c_{k}, g\right]=\left[c_{1}, g\right]^{a_{1}} \cdots\left[c_{k}, g\right]^{a_{k}}
$$

com $a_{1}, \ldots, a_{k} \in G$. Por um lado, se $c_{i} \in X$ então $\left[c_{i}, g\right]^{a_{i}}=\left[c_{i}^{a_{i}}, g^{a_{i}}\right]$ é um comutador de peso $n+1$, pois $c_{i}^{a_{i}} \in X^{a_{i}}=X$. Por outro lado, se $c_{i} \in X^{-1}$ então $c_{i}=d_{i}^{-1}$, onde $d_{i} \in X$. Logo, pela fórmula (1.2.8),

$$
\begin{aligned}
{\left[c_{i}, g\right]^{a_{i}} } & =\left[d_{i}^{-1}, g\right]^{a_{i}}=\left(\left(\left[d_{i}, g\right]^{-1}\right)^{d_{i}^{-1}}\right)^{a_{i}} \\
& =\left(\left[d_{i}, g\right]^{-1}\right)^{d_{i}^{-1} a_{i}}=\left[d_{i}^{\left(d_{i}^{-1} a_{i}\right)}, g^{\left(d_{i}^{-1} a_{i}\right)}\right]^{-1} .
\end{aligned}
$$

Logo, $\left[c_{i}, g\right]^{a_{i}}$ é o inverso de um comutador de peso $n+1$, porque $d_{i}^{\left(d_{i}^{-1} a_{i}\right)} \in X^{\left(d_{i}^{-1} a_{i}\right)}=X$. Portanto, os fatores do produto em (1.3.6) são comutadores de peso $n+1$ ou inversos de comutadores de peso $n+1$ e, assim, $\gamma_{n+1}(G)=\left\langle\left[g_{1}, \ldots, g_{n+1}\right] \mid g_{1}, \ldots, g_{n+1} \in G\right\rangle$. Isto termina a indução e a proposição está provada.

Teorema 1.3.10. Seja G um grupo m-gerado. Então, para todo $k \geq 1$, o quociente $\gamma_{k}(G) / \gamma_{k+1}(G)$ é $\left(m^{k}\right)$-gerado. 
DemonstraçÃo. Seja $G=\langle X\rangle$, com $|X|=m$. Vamos usar indução sobre $k$. Note que $\gamma_{1}(G) / \gamma_{2}(G)$ é $m$-gerado. Agora suponha o resultado vale para $k$. Deve existir um subconjunto $Y \subseteq \gamma_{k}(G)$, com $|Y|=m^{k}$, tal que $\gamma_{k}(G) / \gamma_{k+1}(G)=\left\langle y \gamma_{k+1}(G) \mid y \in Y\right\rangle$. Assim, dado $h \in \gamma_{k}(G)$, temos que $h \gamma_{k+1}(G)=y_{1} \cdots y_{s} \gamma_{k+1}(G)$, com $y_{1}, \ldots, y_{s} \in Y \cup Y^{-1}$. Então $h=y_{1} \cdots y_{s} z$, com $z \in \gamma_{k+1}(G)$.

Por definição, $\gamma_{k+1}(G)=\left[\gamma_{k}(G), G\right]=\left\langle[h, g] \mid h \in \gamma_{k}(G), g \in G\right\rangle$ e, assim, $\gamma_{k+1}(G) / \gamma_{k+2}(G)$ é gerado pelos elementos $[h, g] \gamma_{k+2}(G)$, com $h \in \gamma_{k}(G)$ e $g \in G$. Como $h=y_{1} \cdots y_{s} z, \operatorname{com} y_{1}, \ldots, y_{s} \in Y \cup Y^{-1} \mathrm{e}$ $z \in \gamma_{k+1}(G)$, aplicando a fórmula (1.2.3), temos que

$$
[h, g]=\left[y_{1} \cdots y_{s} z, g\right]=\left[y_{1}, g\right]^{e_{2}} \cdots\left[y_{s-1}, g\right]^{e_{s}}\left[y_{s}, g\right]^{z}[z, g]
$$

onde $e_{2}=y_{2} \cdots y_{s} z, \ldots, e_{s}=y_{s} z$. Agora, lembrando que $a^{b}=a[a, b]$, para quaisquer $a, b \in G$, temos que

$$
[h, g]=\left[y_{1}, g\right]\left[y_{1}, g, e_{2}\right] \cdots\left[y_{s-1}, g\right]\left[y_{s-1}, g, e_{s}\right]\left[y_{s}, g\right]\left[y_{s}, g, z\right][z, g] .
$$

Portanto, no quociente $\gamma_{k+1}(G) / \gamma_{k+2}(G)$ temos que

$$
[h, g] \gamma_{k+2}(G)=\left[y_{1}, g\right] \cdots\left[y_{s}, g\right] \gamma_{k+2}(G)
$$

Como $g=x_{1} \cdots x_{t}$, com $x_{1}, \ldots, x_{t} \in X \cup X^{-1}$, da identidade (1.2.4) segue que, para cada $1 \leq i \leq s$,

$$
\left[y_{i}, g\right]=\left[y_{i}, x_{1} \cdots x_{t}\right]=\left[y_{i}, x_{t}\right]\left[y_{i}, x_{t-1}\right]^{x_{t}} \cdots\left[y_{i}, x_{1}\right]^{x_{2} \cdots x_{t}} .
$$

Portanto, no quociente $\gamma_{k+1}(G) / \gamma_{k+2}(G)$ temos, para cada $1 \leq i \leq s$,

$$
\left[y_{i}, g\right] \gamma_{k+2}(G)=\left[y_{i}, x_{t}\right] \cdots\left[y_{i}, x_{1}\right] \gamma_{k+2}(G)
$$


Ou seja, o elemento $[h, g] \gamma_{k+2}(G)$ é um produto de elementos da forma $\left[y_{i}, x_{j}\right] \gamma_{k+2}(G)$, com $y_{i} \in Y \cup Y^{-1}, x_{j} \in X \cup X^{-1}$. Concluímos que $\gamma_{k+1}(G) / \gamma_{k+2}(G)=\left\langle[u, v] \gamma_{k+2}(G) \mid u \in Y \cup Y^{-1}, v \in X \cup X^{-1}\right\rangle$.

Para todo $y \in Y$ e todo $x \in X$, segue das identidades (1.2.7) e (1.2.8) que $\left[y, x^{-1}\right]=[x, y]^{\left(x^{-1}\right)}=[y, x]^{-1}\left[x, y, x^{-1}\right]$ e, analogamente, $\left[y^{-1}, x\right]=[x, y]^{\left(y^{-1}\right)}=[y, x]^{-1}\left[x, y, y^{-1}\right]$. Podemos mostrar ainda que $\left[y^{-1}, x^{-1}\right]=\left[x^{-1}, y\right]^{y^{-1}}=[y, x]^{y^{-1} x^{-1}}=[y, x]\left[y, x, y^{-1} x^{-1}\right]$. Então,

$$
\begin{aligned}
& {\left[y, x^{-1}\right] \gamma_{k+2}(G)=[y, x]^{-1} \gamma_{k+2}(G),} \\
& {\left[y^{-1}, x\right] \gamma_{k+2}(G)=[y, x]^{-1} \gamma_{n+2}(G),} \\
& {\left[y^{-1}, x^{-1}\right] \gamma_{k+2}(G)=[y, x] \gamma_{k+2}(G) .}
\end{aligned}
$$

De forma que, $\gamma_{k+1}(G) / \gamma_{k+2}(G)=\left\langle[y, x] \gamma_{k+2}(G) \mid y \in Y, x \in X\right\rangle$. Isto quer dizer que $\gamma_{k+1}(G) / \gamma_{k+2}(G)$ é $\left(m^{k+1}\right)$-gerado e a indução está concluída.

Corolário 1.3.11. Seja $G$ um grupo m-gerado tal que $\gamma_{r}(G)$ tem ordem finita $n$, para algum $r \geq 1$. Então

(i) todos os termos da série central descendente de $G$ são finitamente gerados;

(ii) para todo $1 \leq j \leq r-1$, temos que $\gamma_{r-j}(G)$ é gerado por $\left\lfloor\log _{2}(n)\right\rfloor+m^{r-1}+\cdots+m^{r-j}$ elementos.

DemonstraÇÃo. (ii) Argumentamos por indução sobre $j$. Observe que, pela Proposição 1.1.4, $\gamma_{r}(G)$ é $\left\lfloor\log _{2}(n)\right\rfloor$-gerado e, pelo Teorema 1.3.10, $\gamma_{r-1}(G) / \gamma_{r}(G)$ é $\left(m^{r-1}\right)$-gerado. Logo, pela Proposição 1.1.1, $\gamma_{r-1}(G)$ pode ser gerado por $\left\lfloor\log _{2}(n)\right\rfloor+m^{r-1}$ elementos. Isto estabelece o caso $j=1$. Agora suponha, por indução sobre $j$, que $\gamma_{r-j}(G)$ é gerado por $\left\lfloor\log _{2}(n)\right\rfloor+m^{r-1}+\cdots+m^{r-j}$ elementos. Pelo 
Teorema 1.3.10, temos que $\gamma_{r-j-1}(G) / \gamma_{r-j}(G)$ é $\left(m^{r-j-1}\right)$-gerado. Assim, segue da Proposição 1.1.1 que $\gamma_{r-j-1}(G)$ pode ser gerado por $\left\lfloor\log _{2}(n)\right\rfloor+m^{r-1}+\cdots+m^{r-j}+m^{r-j-1}$ elementos. Isto conclui a indução.

(i) Claramente $\gamma_{r+k}(G)$ é finitamente gerado, para todo $k \geq 0$, pois $\gamma_{r}(G)$ é finito. Disso e da parte (ii) concluímos que todos os termos da série central descendente de $G$ são finitamente gerados.

\subsection{Grupos solúveis e nilpotentes}

Um grupo G é solúvel se possui uma série abeliana, isto é, uma série $G=G_{0} \geq G_{1} \geq \cdots \geq G_{n}=1$ onde $G_{i+1}$ é normal em $G_{i}$ e $G_{i} / G_{i+1}$ é abeliano, para todo $0 \leq i \leq n-1$.

Dado um grupo $G$, chamamos de série derivada a seguinte série $G=G^{(0)} \geq G^{(1)} \geq G^{(2)} \geq \cdots$, onde $G^{(i+1)}=\left[G^{(i)}, G^{(i)}\right]$, para todo $i \geq 0$.

ProposiçÃo 1.4.1. Se $G=G_{0} \geq G_{1} \geq \cdots \geq G_{n}=1$ é uma série abeliana de um grupo solúvel $G$, então $G^{(i)} \leq G_{i}$, para todo $0 \leq i \leq n$. Em particular, $G^{(n)}=1$.

Demonstração. Vamos provar por indução sobre $i$. Para $i=0$ o resultado é óbvio. Agora suponha que $G^{(i)} \leq G_{i}$. Como $G_{i} / G_{i+1}$ é abeliano, a Proposição 1.3.1 garante que $\left(G_{i}\right)^{\prime} \leq G_{i+1}$. Assim, temos que $G^{(i+1)}=\left(G^{(i)}\right)^{\prime} \leq\left(G_{i}\right)^{\prime} \leq G_{i+1}$. Isto conclui a indução e mostra que $G^{(i)} \leq G_{i}$, para todo $0 \leq i \leq n$. Em particular, $G^{(n)} \leq G_{n}=1$.

O comprimento derivado de um grupo solúvel $G$ é o menor inteiro $d$ tal que $G^{(d)}=1$.

Um grupo $G$ é metabeliano se é solúvel de comprimento derivado menor ou igual a 2. Ou seja, $G$ é metabeliano se possui um subgrupo normal abeliano cujo quociente é abeliano. 
Um grupo $G$ é nilpotente se possui uma série central, isto é, uma série $G=G_{0} \geq G_{1} \geq \cdots \geq G_{n}=1$ onde $G_{i+1}$ é normal em $G_{i}$ e $G_{i} / G_{i+1}$ é abeliano, para todo $0 \leq i \leq n-1$. Pode-se mostrar que $G$ é nilpotente se, e somente se, $\gamma_{n+1}(G)=1$ para algum inteiro $n$. Se $c$ é o menor inteiro tal que $\gamma_{c+1}(G)=1$, dizemos que $G$ é nilpotente de classe $c$.

Proposição 1.4.2. Todo grupo nilpotente é solúvel.

DemonstraÇÃo. Seja $G$ nilpotente de classe $c$. Mostraremos que $G^{(n)} \leq \gamma_{n+1}(G)$, para todo $n \geq 0$. Em particular $G^{(c)} \leq \gamma_{c+1}(G)=1$. O caso $n=0$ é trivial. Agora, supondo que $G^{(n)} \leq \gamma_{n+1}(G)$, temos que $G^{(n+1)}=\left[G^{(n)}, G^{(n)}\right] \leq\left[\gamma_{n+1}(G), G\right]=\gamma_{n+2}(G)$. Isto prova a afirmação.

Observamos que a recíproca da Proposição 1.4.2 não é verdadeira, como mostra o grupo $S_{3}=\left\langle a, b \mid a^{3}=b^{2}=1, a^{b}=a^{-1}\right\rangle$. Nesse grupo, a série derivada é $1 \leq\langle a\rangle \leq S_{3}$. Assim, $S_{3}$ é metabeliano mas não é nilpotente, pois $\gamma_{n}\left(S_{3}\right)=\langle a\rangle$, para todo $n \geq 2$.

\section{5. $p$-grupos extra especiais}

O objetivo desta seção é construir um exemplo de um p-grupo extra especial infinito. A existência de grupos desse tipo mostra que a recíproca do Teorema de Schur não vale em geral, como veremos no Capítulo 2. Estes grupos também são importantes para a construção dos contra exemplos para a recíproca do Teorema de Baer.

Seguindo P. Hall e Higman [12, p. 15] damos a seguinte definição.

DEFiniÇÃo 1.5.1. Sejam $p$ um número primo e $G$ um $p$-grupo. O grupo $G$ é um $p$-grupo especial se $G$ é um $p$-grupo abeliano elementar 
ou se $G$ é um $p$-grupo de classe 2 tal que $G^{\prime}=Z(G)$ e o quociente $G / G^{\prime}$ é um $p$-grupo abeliano elementar. Dizemos que $G$ é um $p$-grupo extra especial se $G$ é um $p$-grupo especial não abeliano tal que $G^{\prime}=Z(G)$ é cíclico de ordem $p$.

Sendo assim, um grupo p-grupo $G$ é extra especial quando $G^{\prime}$ e $Z(G)$ coincidem e tem ordem $p$ e, além disso, $G / G^{\prime}$ é um $p$-grupo abeliano elementar.

Seja $G$ um $p$-grupo não abeliano de ordem $p^{3}$. O centro $G$ não pode ser trivial, pois $G$ é um p-grupo. Então, pelo Teorema de Lagrange $Z(G)$ tem ordem $p$ ou $p^{2}$. Se $Z(G)$ tivesse ordem $p^{2}$, o quociente $G / Z(G)$ teria ordem $p$ e $G$ seria abeliano. Portanto, $Z(G)$ tem ordem $p$ e o grupo $G / Z(G)$ tem ordem $p^{2}$. Por ter ordem $p^{2}$, o quociente $G / Z(G)$ é abeliano e deve ser isomorfo a $C_{p^{2}}$ ou a $C_{p} \times C_{p}$, onde $C_{n}$ denota o grupo cíclico de ordem $n$. Como $G$ não é abeliano, $G / Z(G)$ deve ser isomorfo a $C_{p} \times C_{p}$. Note que $G^{\prime} \leq Z(G)$, pois $G / Z(G)$ é abeliano. Logo, $G^{\prime}=Z(G)$ pois $G^{\prime} \neq 1$. Isto mostra $G$ é extra especial.

Os únicos 2-grupos não abelianos de ordem $2^{3}$ são, a menos de isomorfismos, o diedral $D_{4}$ e o grupo dos quatérnions $Q_{8}$.

Quando $p$ é um primo ímpar, definimos dois grupos de ordem $p^{3}$ :

$$
\begin{gathered}
\left.M(p)=\langle x, y, z| x^{p}=y^{p}=z^{p}=1,[x, z]=[y, z]=1 \text { e }[x, y]=z\right\rangle \\
\left.M_{3}(p)=\langle x, y| x^{p^{2}}=y^{p}=1 \text { e } x^{y}=x^{1+p}\right\rangle .
\end{gathered}
$$

Dado um p-grupo $G$ lembramos que $\Omega_{1}(G)=\left\langle g \in G \mid g^{p}=1\right\rangle$. Note que $M(p)$ é de classe 2 e $\Omega_{1}(M(p))=M(p)$. Assim, $M(p)$ possui expoente $p$. Por sua vez, $M_{3}(p)$ é de classe 2 mas $\Omega_{1}\left(M_{3}(p)\right)$ é abeliano 
elementar de ordem $p^{2}$ e, assim, $\Omega_{1}\left(M_{3}(p)\right) \neq \Omega_{1}\left(M_{3}(p)\right)$. Em particular, $M(p)$ e $M_{3}(p)$ não são isomorfos embora ambos possuam ordem $p^{3}$. Para mais detalhes sobre esses dois grupos veja [8, pp. 190-203].

O resultado a seguir fornece uma classificação dos p-grupos não abelianos de ordem $p^{3}$.

Teorema 1.5.2. [8, §5 Theorem 5.1] Seja G um p-grupo não abeliano de ordem $p^{3}$. Então $G$ é extra especial. Se $p=2$ então $G$ é isomorfo a $D_{4}$ ou $Q_{3}$. Se $p>2$ então $G$ é isomorfo a $M_{3}(p)$ ou $M(p)$.

Seja $\left\{G_{i} \mid i \in I\right\}$ uma família de grupos. O produto cartesiano (ou produto irrestrito) de $\left\{G_{i} \mid i \in I\right\}$ é o grupo

$$
\mathrm{Cr}_{i \in I} G_{i}=\left\{f: I \rightarrow \bigcup_{i \in I} G_{i} \mid f(i) \in G_{i}, \forall i \in I\right\}
$$

onde a operação é tal que, dados $f, g \in \mathrm{Cr}_{i \in I} G_{i}$, o produto $f g$ é a função tal que $(f g)(i)=f(i) g(i)$, para todo $i \in I$. Note que a operação está bem definida, pois $f(i), g(i) \in G_{i}$, para todo $i \in I$. Em geral denotamos um elemento do produto cartesiano por $\left(g_{i}\right)$, sendo que $g_{i} \in G_{i}$, e pensamos nesses elementos como $I$-uplas. Com essa notação, a operação do grupo é definida como $\left(g_{i}\right)\left(h_{i}\right)=\left(g_{i} h_{i}\right)$.

Estamos interessados no seguinte subgrupo do produto cartesiano:

$$
\prod_{i \in I} G_{i}=\left\{\left(g_{i}\right) \in \mathrm{Cr}_{i \in I} G_{i} \mid\left\{i \in I \mid g_{i} \neq 1\right\} \text { é finito }\right\} .
$$

Este subgrupo é chamado de produto direto (ou restrito). Observe que $\prod_{i \in I} G_{i}$ é o conjunto dos elementos $\left(g_{i}\right)$ tais que $g_{i}=1$ exceto para um número finito de índices.

É fácil ver que se $H_{i} \leq G_{i}$, para todo $i \in I$, então $\prod_{i \in I} H_{i} \leq \prod_{i \in I} G_{i}$ e se $H_{i}$ é um subgrupo normal de $G_{i}$, para todo $i \in I$, então $\prod_{i \in I} H_{i}$ é um subgrupo normal de $\prod_{i \in I} G_{i}$. Podemos mostrar ainda que

$$
\left(\prod_{i \in I} G_{i}\right)^{\prime}=\prod_{i \in I} G_{i}^{\prime}
$$




$$
Z\left(\prod_{i \in I} G_{i}\right)=\prod_{i \in I} Z\left(G_{i}\right)
$$

Agora estamos em condições de construir um p-grupo extra especial infinito. Sejam $p$ um primo e $G$ um $p$-grupo não abeliano de ordem $p^{3}$. Considere o produto direto $\prod_{i \in \mathbb{N}} G$ e o subgrupo normal

$$
N=\left\{\left(g_{i}\right) \in \prod_{i \in \mathbb{N}} Z(G) \mid \prod_{i \in \mathbb{N}} g_{i}=1\right\} .
$$

Note que, para todo $\left(g_{i}\right) \in \prod_{i \in \mathbb{N}} G$, o produto $\prod_{i \in \mathbb{N}} g_{i}$ faz sentido porque $\left\{i \in I \mid g_{i} \neq 1\right\}$ é finito.

Definimos $E=\left(\prod_{i \in \mathbb{N}} G\right) / N$ e afirmamos que $E$ é um $p$-grupo extra especial infinito. Primeiramente, é possível mostrar que

$$
Z(E)=\left(\prod_{i \in \mathbb{N}} Z(G)\right) / N \cong Z(G)
$$

que tem ordem $p$, pois $G$ é extra especial. Agora notamos que

$$
E / Z(E) \cong\left(\prod_{i \in \mathbb{N}} G\right) /\left(\prod_{i \in \mathbb{N}} Z(G)\right) \cong \prod_{i \in \mathbb{N}}(G / Z(G))
$$

que é abeliano elementar infinito. Finalmente, observamos que

$$
E^{\prime}=\left(\prod_{i \in \mathbb{N}} G\right)^{\prime} / N=\left(\prod_{i \in \mathbb{N}} G^{\prime}\right) / N=\left(\prod_{i \in \mathbb{N}} Z(G)\right) / N=Z(E) .
$$

Isto mostra que $E$ é um p-grupo extra especial infinito, como queríamos.

\subsection{Ação de grupos}

Seja $G$ um grupo e $\Omega$ um conjunto não vazio. Uma ação de $G$ sobre $\Omega$ é uma função $\Psi: \Omega \times G \longrightarrow \Omega$ tal que $\Psi(x, g h)=\Psi(\Phi(x, g), h)$ e $\Psi(x, 1)=x$, quaisquer que sejam $x \in \Omega$ e $g, h \in G$.

Em geral, denotamos $\Psi(x, g)$ por $x g$, de forma que as relações acima se reescrevem como $x(g h)=(x g) h$ e $x 1=x$, quaisquer que sejam $x \in \Omega$ e $g, h \in G$.

Para cada elemento $g \in G$, a aplicação $x \mapsto x g$ é uma permutação em $\Omega$. De fato, se $x g=y g, \operatorname{com} x, y \in G$, então $(x g) g^{-1}=(y g) g^{-1} \mathrm{e}$ $x=y$. Agora, dado $x \in \Omega, x=x 1=x\left(g^{-1} g\right)=\left(x g^{-1}\right) g=y g$, onde 
$y=x g^{-1} \in \Omega$. Portanto, $x \mapsto x g$ é injetiva e sobrejetiva. Chamemos essa permutação de $\sigma_{g}$.

Note que, $\sigma_{g h}: x \mapsto x(g h)$ e a composta $\sigma_{g} \sigma_{h}: x \mapsto(x g) h$ são idênticas. Logo, a ação de $G$ sobre $\Omega$ determina um homomorfismo $\sigma: G \rightarrow \operatorname{Sym}(\Omega)$ tal que $g \mapsto \sigma_{g}$.

Reciprocamente, dado um homomorfismo $\sigma: G \rightarrow \operatorname{Sym}(\Omega)$ tal que $g \mapsto \sigma_{g}$, a aplicação $\Psi:(x, g) \longmapsto(x) \sigma_{g}$ é uma ação de $G$ sobre $\Omega$. Um homomorfismo $\sigma: G \rightarrow \operatorname{Sym}(\Omega)$ é chamado de uma representação permutacional de $G$ sobre $\Omega$. A representação é dita fiel se $\operatorname{Ker}(\sigma)=1$, pois nesse caso $G$ é isomorfo a um subgrupo de $\operatorname{Sym}(\Omega)$.

Um dos exemplos de ação de grupo que mais usamos nesta dissertação é o da ação de um grupo $G$ no conjunto das classes laterais de um subgrupo de $G$. Dado um subgrupo $H$ de $G$, denote por $\mathfrak{R}$ o conjunto das classes laterais à direita de $H$ em $G$. A função de $\mathfrak{R} \times G \rightarrow \mathfrak{R}$ tal que $(H x, g) \mapsto H x g$ é uma ação de $G$ sobre $\mathfrak{R}$. Esta ação apareceu naturalmente na demonstração do Teorema 1.1.8 de Schreier e voltará a aparecer mais adiante na seção 1.7 onde definiremos o transfer.

Outro exemplo de ação de grupo que usaremos repetidamente é o da ação por conjugação de um subgrupo $H$ de um grupo $G$ no grupo quociente $G / N$, onde $N$ é um subgrupo normal de $G$. A função de $G / N \times H \rightarrow G / N$ tal que $(x N, h) \mapsto x^{h} N$ é uma ação de $H$ sobre $G / N$. A essa ação corresponde um homomorfismo $\sigma: H \rightarrow \operatorname{Sym}(G / N)$ tal que $\sigma: h \mapsto \sigma_{h}$, onde $\sigma_{h}(x N)=x^{h} N$, para todo $x N \in G / N$.

Listamos a seguir uma série de resultados que serão usados neste trabalho, especialmente no Capítulo 3. Notamos que o conceito de ação de grupos proporciona uma interpretação mais adequada dos resultados que apresentaremos aqui. 
Proposição 1.6.1. Seja $N$ um subgrupo normal de um grupo $G$. A aplicação a definida abaixo é um homomorfismo.

$$
\begin{aligned}
\alpha: G / C_{G}(N) & \longrightarrow \operatorname{Aut}(N) \\
g C_{G}(N) & \longmapsto\left(x \mapsto x^{g}\right)
\end{aligned}
$$

DemonstraÇÃo. Primeiro verificamos que $\alpha$ está bem definida. Se $g, h \in G$ são tais que $g C_{G}(N)=h C_{G}(N)$ então $h=g c$, para algum $c \in C_{G}(N)$. Como $N$ é normal em $G$, temos que $x^{h}=x^{g c}=$ $\left(x^{g}\right)^{c}=x^{g}$, para todo $x \in N$, ou seja, $\alpha\left(h C_{G}(N)\right)=\alpha\left(g C_{G}(N)\right)$. Agora vejamos que $\alpha$ é um homomorfismo. Dados $g C_{G}(N)$ e $h C_{G}(N)$ elementos arbitrários de $G / C_{G}(N)$, temos que $x^{g h}=\left(x^{g}\right)^{h}$, para todo $x \in N$. Então $\alpha\left(g h C_{G}(N)\right)(x)=\alpha\left(g C_{G}(N)\right) \circ \alpha\left(h C_{G}(N)\right)(x)$, para todo $x \in N$. Assim, $\alpha\left(g h C_{G}(N)\right)=\alpha\left(g C_{G}(N)\right) \circ \alpha\left(h C_{G}(N)\right)$ e isso mostra que $\alpha$ é de fato um homomorfismo.

Considere o produto semidireto $N \rtimes_{\alpha}\left(G / C_{G}(N)\right)$. Como é usual, identificamos $N \times 1$ com $N$ e $1 \times\left(G / C_{G}(N)\right) \operatorname{com} G / C_{G}(N)$ e denotamos $\alpha\left(g C_{G}(N)\right)(x)$ por $x^{\bar{g}}$, para todo $x \in N$ e todo $g \in G$. Vamos calcular o subgrupo comutador de $N$ e $G / C_{G}(N)$.

$$
\left.\left[N, G / C_{G}(N)\right]=\left\langle x^{-1} x^{\bar{g}}\right| x \in N \text { e } g \in G\right\rangle
$$

Observe que $x^{\bar{g}}=x^{g}$ então $x^{-1} x^{\bar{g}}=[x, g]$, para todo $x \in N$ e todo $g \in G$. Portanto, $\left[N, G / C_{G}(N)\right]=[N, G]$. Vamos enunciar esse fato como uma proposição, pois precisaremos dele mais adiante.

ProposiçÃo 1.6.2. Seja $N$ um subgrupo normal de um grupo $G$. $\operatorname{Em} N \rtimes_{\alpha}\left(G / C_{G}(N)\right)$ temos que $\left[N, G / C_{G}(N)\right]=[N, G]$. 
DefiniÇÃo 1.6.3. Sejam $N \leq H$ subgrupos de um grupo $G$, sendo $N$ normal em $G$. Se $\bar{X} \subseteq G / N$ é um conjunto não vazio, definimos $C_{H}(\bar{X})=\left\{h \in H \mid x^{h} N=x N\right.$, para todo $\left.x N \in \bar{X}\right\}$ que é igual ao conjunto $\{h \in H \mid[x, h] \in N$, para todo $x N \in \bar{X}\}$.

Observe que $C_{H}(\bar{X})$ é o subgrupo de $H$ tal que $C_{H}(\bar{X}) / N=C_{H / N}(\bar{X})$.

ProposiçÃo 1.6.4. Sejam $N$ e $M$ subgrupos normais de um grupo $G$ tais que $N \leq M \leq G$. Então o centralizador $C_{G}(M / N)$ é normal em $G$.

Demonstração. Por definição, $C_{H}(M / N)$ é o subgrupo de $G$ tal que $C_{G}(M / N) / N=C_{G / N}(M / N)$. Pela Proposição 1.2.2, $C_{G / N}(M / N)$ é normal em $G / N$, pois $M / N$ é normal em $G / N$. Portanto, pelo Teorema da Correspondência, $C_{G}(M / N)$ é um subgrupo normal de $G$.

ProposiçÃo 1.6.5. Sejam $N, H$ e $K$ subgrupos de um grupo $G$ tais que $N$ é normal em $G, N \leq H$ e $N \leq K$. Vale a igualdade

$$
\left[K / N: C_{K / N}(H / N)\right]=\left[K: C_{K}(H / N)\right]
$$

Demonstração. Por definição, temos $C_{K / N}(H / N)=C_{K}(H / N) / N$. Então, $\left[K / N: C_{K / N}(H / N)\right]=\left[K / N: C_{K}(H / N) / N\right]$. Assim, usando o terceiro Teorema do Isomorfismo, o resultado segue.

Proposição 1.6.6. Sejam $H$ e $U$ subgrupos de um grupo G. Se U é m-gerado e $[U, H]$ é finito, então o centralizador $C_{H}(U)$ tem índice finito em $H$. Mais precisamente, $\left[H: C_{H}(U)\right] \leq|[U, H]|^{m}$.

DemonstraÇÃo. Seja $U=\left\langle u_{1}, \ldots, u_{m}\right\rangle$. Observe que o centralizador $C_{H}(U)=C_{H}\left(u_{1}\right) \cap \cdots \cap C_{H}\left(u_{m}\right)$. Para todo $u \in U$, temos $\left[H: C_{H}(u)\right]=\left|u^{H}\right|$. Mas $\left|u^{H}\right| \leq|[U, H]|$, pois $\varphi: u^{H} \rightarrow[U, H]$, tal 
que $\varphi\left(u^{h}\right)=u^{-1} u^{h}=[u, h]$, é injetiva. Logo, para cada $1 \leq i \leq m$, tem-se $\left[H: C_{H}\left(u_{i}\right)\right] \leq|[U, H]|$. Segue da Proposição 1.0.2 que

$$
\left[H: C_{H}(U)\right] \leq \prod_{i=1}^{m}\left[H: C_{H}\left(u_{i}\right)\right] \leq|[U, H]|^{m} .
$$

A proposição anterior, como está enunciada, foi encontrada no artigo [7, Lema 2.1]. Mas a ideia usada em sua demonstração já havia aparecido anteriormente, por exemplo, no artigo $[\mathbf{2 2}]$ de B. H. Neumann.

Corolário 1.6.7. Sejam $U$ e $N \leq H$ subgrupos de um grupo $G$, sendo $N$ normal em $G$. Se $U$ é m-gerado e $[H, U] N / N$ tem ordem $n$, então $\left[H: C_{H}(U N / N)\right] \leq n^{m}$.

DemonstraÇÃo. Vamos aplicar a Proposição 1.6.6 ao grupo $G / N$. Como $U$ é $m$-gerado, temos que $U N / N$ é $m$-gerado. Note que o subgrupo comutador $[H / N, U N / N]=[H, U] N / N$, que tem ordem $n$, por hipótese. Então, pela Proposição 1.6.6, $\left[H / N: C_{H / N}(U N / N)\right] \leq n^{m}$. Pela Proposição 1.6.5, esse índice é igual a $\left[H: C_{H}(U N / N)\right]$, o que conclui a demonstração.

\subsection{O homomorfismo transfer}

Seja $G$ um grupo e por comodidade denote $Z=Z(G)$. Suponha que $[G: Z]=n$ e seja $T$ um transversal à direita para $Z$ em $G$. Vamos considerar a ação de $G$ em $G / Z$ por multiplicação à direita. De forma que, a cada elemento $g \in G$ corresponde uma permutação das classes laterais de $Z$ em $G$. Assim, dado $t \in T$, existe um único $t_{g} \in T$ tal que $Z t g=Z t_{g}$. Note que, $\operatorname{tg}_{g}^{-1} \in Z$. Para $g$ fixado e fazendo $t$ percorrer 
$T$, obtemos $n$ elementos da forma $t g t_{g}^{-1}$. Definiremos o transfer (com respeito a $T$ ) como sendo a função $\tau: G \rightarrow Z(G)$ tal que

$$
\tau(g)=\prod_{t \in T} t g t_{g}^{-1}
$$

Note que, como $\operatorname{tgt}_{g}^{-1} \in Z$, a ordem dos fatores do produto acima é irrelevante.

A proposição a seguir mostra que a escolha do transversal $T$ de $Z$ em $G$ também é irrelevante na definição do transfer $\tau$.

ProposiçÃo 1.7.1. Seja $G$ um grupo tal que $[G: Z(G)]$ é finito. $O$ transfer $\tau: G \rightarrow Z(G)$ não depende da escolha do transversal de $Z(G)$ em $G$.

DemonstraÇÃo. Seja $g \in G$ um elemento arbitrário e sejam $T$ e $L$ dois transversais à direita para $Z$ em $G$. Vamos mostrar que

$$
\prod_{t \in T} \operatorname{tg}_{g}^{-1}=\prod_{l \in L} l g l_{g}^{-1}
$$

Existe uma bijeção $\alpha: T \rightarrow L$ tal que $Z t=Z \alpha(t)$, para todo $t \in T$. A existência da bijeção $\alpha$ decorre do fato de que $T$ e $L$ são transversais para $Z$ em $G$. Existe ainda uma função $z: T \rightarrow Z$ tal que

$$
t=z(t) \alpha(t) \quad \text { para todo } t \in T \text {. }
$$

Agora, considerando a ação de $g$ em $G / Z$, para cada $t \in T$ existe $t_{g} \in T$ tal que $Z t g=Z t_{g}$. Além disso, para cada $l \in L$ existe $l_{g} \in L$ tal que $Z l g=Z l_{g}$. Pela definição de $\alpha$, temos $Z t=Z \alpha(t)$. Segue que $Z t g=Z \alpha(t) g$ e, portanto, $Z t_{g}=Z \alpha(t)_{g}$. Concluímos, pela própria definição de $\alpha$, que $\alpha\left(t_{g}\right)=\alpha(t)_{g}$. Segue da equação (1.7.1) que

$$
t_{g}=z\left(t_{g}\right) \alpha\left(t_{g}\right)=z\left(t_{g}\right) \alpha(t)_{g} .
$$


Finalmente, das equações (1.7.1) e (1.7.2) temos que

$$
\prod_{t \in T} \operatorname{tgt}_{g}^{-1}=\prod_{t \in T} z(t) \alpha(t) \cdot g \cdot\left(\alpha(t)_{g}\right)^{-1} z\left(t_{g}\right)^{-1}
$$

Como $z(t), z\left(t_{g}\right) \in Z$, segue que

$$
\prod_{t \in T} t g t_{g}^{-1}=\prod_{t \in T} \alpha(t) g\left(\alpha(t)_{g}\right)^{-1} \cdot \prod_{t \in T} z(t) z\left(t_{g}\right)^{-1}
$$

Notando que $t_{g}$ percorre todo o conjunto $T$, conforme $t$ varia em $T$, temos que $\prod_{t \in T} z(t) z\left(t_{g}\right)^{-1}=1$. Além disso, como $\alpha: T \rightarrow L$ é uma bijeção, temos que $\prod_{t \in T} \alpha(t) g\left(\alpha(t)_{g}\right)^{-1}=\prod_{l \in L} l g l_{g}^{-1}$. Assim, a equação (1.7.3) pode ser reescrita como

$$
\prod_{t \in T} t g t_{g}^{-1}=\prod_{l \in L} l g l_{g}^{-1} .
$$

Isto mostra que a escolha do transversal não altera o valor $\tau(g)$. Como $g$ é um elemento arbitrário de $G$, o resultado está demonstrado.

Proposição 1.7.2. Seja $G$ um grupo tal que $[G: Z(G)]$ é finito. $O$ transfer $\tau: G \rightarrow Z(G)$ é um homomorfismo.

Demonstração. Sejam $g, h \in G$ elementos arbitrários e $T$ um transversal à direita para $Z$ em $G$. Para cada $t \in T$, por um lado temos que $Z t g h=Z t_{g h}$ e, por outro lado, $Z t g h=Z t_{g} h=Z\left(t_{g}\right)_{h}$. Então $t_{g h}=\left(t_{g}\right)_{h}$. Assim, $\operatorname{tgh}\left(t_{g h}\right)^{-1}=\operatorname{tgh}\left(\left(t_{g}\right)_{h}\right)^{-1}=\operatorname{tg}_{g}^{-1} \cdot t_{g} h\left(\left(t_{g}\right)_{h}\right)^{-1} \mathrm{e}$, portanto,

$$
\tau(g h)=\prod_{t \in T} \operatorname{tgh}\left(t_{g h}\right)^{-1}=\prod_{t \in T} \operatorname{tg} t_{g}^{-1} \cdot t_{g} h\left(\left(t_{g}\right)_{h}\right)^{-1}
$$

Lembrando que $\operatorname{tg}_{g}^{-1} \in Z$ e que $t_{g} h\left(\left(t_{g}\right)_{h}\right)^{-1} \in Z$, concluímos que

$$
\tau(g h)=\prod_{t \in T} t g t_{g}^{-1} \prod_{t \in T} t_{g} h\left(\left(t_{g}\right)_{h}\right)^{-1}=\tau(g) \tau(h)
$$


Note que, usamos o fato de $t \mapsto t_{g}$ ser uma bijeção em $T$, para ver que $\prod_{t \in T} t_{g} h\left(\left(t_{g}\right)_{h}\right)^{-1}=\tau(h)$.

O transfer, como definimos aqui, é um caso particular de uma definição mais geral. Seja $G$ um grupo e $H$ um subgrupo de índice finito $n$ em $G$. Dado um transversal à direita $T$ para $H$ em $G$, temos que, para $t \in T$ e $g \in G$, existe um único $t_{g} \in T$ tal que $H t g=H t_{g}$. Assim, $\operatorname{tg}_{g}^{-1} \in H$. Suponha que $\pi: H \rightarrow A$ é um homomorfismo de $H$ em algum grupo abeliano $A$. O transfer de $\pi$ é a função $\tau: G \rightarrow A$ tal que

$$
\tau(g)=\prod_{t \in T}\left(\operatorname{tg}_{g}^{-1}\right)^{\pi}
$$

Uma vez que $A$ é abeliano, a ordem dos fatores não importa no produto acima. Nessa definição mais geral também é possível mostrar que $\tau$ não depende do transversal $T$ e que $\tau$ é um homomorfismo (veja [25, pp. 285-287]). Note que no nosso caso $H=Z(G)$ e $\pi$ é a identidade em $Z(G)$.

Na seção 2.1 voltaremos a falar do transfer provando outros resultados úteis para a demonstração do Teorema de Schur.

\subsection{Produto tensorial de grupos abelianos}

Se $G$ e $H$ são dois grupos abelianos, definimos o produto tensorial de $G$ e $H$, denotado por $G \otimes H$, como sendo o grupo abeliano gerado pelo conjunto $\{g \otimes h \mid g \in G, h \in H\}$ sujeito às relações

$$
\begin{aligned}
& g_{1} g_{2} \otimes h_{1}=\left(g_{1} \otimes h_{1}\right)\left(g_{2} \otimes h_{1}\right), \\
& g_{1} \otimes h_{1} h_{2}=\left(g_{1} \otimes h_{1}\right)\left(g_{1} \otimes h_{2}\right) .
\end{aligned}
$$

Isto é, $G \otimes H$ é isomorfo a $F / R$, onde $F$ é o grupo abeliano livre no conjunto $G \times H$ e $R$ é o subgrupo de $F$ gerado pelos elementos do 
tipo $\left(g_{1} g_{2}, h\right)\left(g_{1}, h\right)\left(g_{2}, h\right)$ e $\left(g, h_{1} h_{2}\right)\left(g, h_{1}\right)\left(g, h_{2}\right)$, com $g, g_{1}, g_{2} \in G$ e $h, h_{1}, h_{2} \in H$.

Dizemos que uma aplicação $\varphi: G \times H \rightarrow K$ é bilinear se, para quaisquer $g_{1}, g_{2} \in G$ e $h_{1}, h_{2} \in H, \varphi\left(g_{1} g_{2}, h_{1}\right)=\varphi\left(g_{1}, h_{1}\right) \varphi\left(g_{2}, h_{1}\right)$ e $\varphi\left(g_{1}, h_{1} h_{2}\right)=\varphi\left(g_{1}, h_{1}\right) \varphi\left(g_{1}, h_{2}\right)$. Pode-se mostrar que, dados um grupo $K$ e uma aplicação bilinear $\varphi: G \times H \rightarrow K$, existe um homomorfismo $\rho: G \otimes H \rightarrow K$ tal que $\rho(g \otimes h)=\varphi(g, h)$, para todo $g \in G$ e todo $h \in H$.

Proposição 1.8.1. Sejam A, B e $C$ grupos abelianos. Então

$$
A \otimes(B \times C) \cong(A \otimes B) \times(A \otimes C) .
$$

Veja [28, Theorem 8.87] para a demonstração dessa proposição.

Denotamos por $C_{n}$ o grupo cíclico finito de ordem $n$.

LEMA 1.8.2. [28, Exercise 8.48] Sejam $m \geq 1$ e $n \geq 1$. Então $C_{m} \otimes C_{n} \cong C_{d}$, onde $d=\operatorname{mdc}(m, n)$.

Proposição 1.8.3. Sejam $A=\prod_{i=1}^{r} C_{m_{i}}$ e $B=\prod_{j=1}^{s} C_{n_{j}}$ dois grupos abelianos finitos. Então $|A \otimes B| \leq|A|^{|B|}$.

Demonstração. Pela Proposição 1.8.1 temos que

$$
A \otimes B \cong \prod_{j=1}^{s}\left(\prod_{i=1}^{r} C_{m_{i}} \otimes C_{n_{j}}\right) .
$$

Segue do Lema 1.8.2 que

$$
A \otimes B \cong \prod_{j=1}^{s}\left(\prod_{i=1}^{r} C_{\mathrm{mdc}\left(m_{i}, n_{j}\right)}\right) .
$$


Como $\left|C_{\operatorname{mdc}\left(m_{i}, n_{j}\right)}\right| \leq\left|C_{m_{i}}\right|$, temos que

$$
|A \otimes B| \leq \prod_{j=1}^{s}\left(\prod_{i=1}^{r}\left|C_{m_{i}}\right|\right)=\prod_{j=1}^{s}(|A|)=|A|^{s} \leq|A|^{|B|}
$$




\section{CAPíTULO 2}

\section{Os Teoremas de Schur, Baer e Hall}

Neste capítulo demonstramos os teoremas clássicos de Schur, Baer e Hall e fornecemos algumas recíprocas dos Teoremas de Schur e de Baer para certas classes de grupos.

\subsection{Teorema de Schur como aplicação do transfer}

O objetivo desta seção é mostrar que se $G$ é um grupo tal que $[G: Z(G)]$ é finito então $G^{\prime}$ é finito. Veremos inicialmente que, se $[G: Z(G)]$ é finito então $G^{\prime}$ é finitamente gerado. Os resultados desta seção foram retirados de [25, pp. 285-287].

Lema 2.1.1. Seja $G$ um grupo. Se $[G: Z(G)]=n$ então $G^{\prime}$ pode ser gerado por $(n-1)(n-2) / 2$ elementos.

DemonstraÇÃo. Seja $T=\left\{t_{1}, \ldots t_{n}\right\}$ um transversal à direita para $Z(G)$ em $G$, e assuma, sem perda de generalidade, que $t_{1}=1$. Dados $g_{1}, g_{2} \in G$, escrevemos $g_{1}=z_{1} t_{i}$ e $g_{2}=z_{2} t_{j}$, com $z_{1}, z_{2} \in Z(G)$ e $t_{i}, t_{j} \in\left\{t_{1}, \ldots, t_{n}\right\}$. De forma que $\left[g_{1}, g_{2}\right]=\left[z_{1} t_{i}, z_{2} t_{j}\right]=\left[t_{i}, t_{j}\right]$, pois $z_{1}, z_{2} \in Z(G)$. Portanto, $\left\{\left[g_{1}, g_{2}\right] \mid g_{1}, g_{2} \in G\right\}=\left\{\left[t_{i}, t_{j}\right] \mid t_{i}, t_{j} \in T\right\}$.

Como $\left[t_{i}, t_{i}\right]=1$ e $\left[t_{i}, t_{j}\right]=\left[t_{j}, t_{i}\right]^{-1}$, para quaisquer $t_{i}, t_{j} \in T$, segue que $G^{\prime}=\left\langle\left[t_{i}, t_{j}\right] \mid 1 \leq i<j \leq n\right\rangle$. Notando que $\left[t_{1}, t_{j}\right]=1$, para todo $j \geq 1$, segue que $G^{\prime}=\left\langle\left[t_{i}, t_{j}\right] \mid 2 \leq i<j \leq n\right\rangle$. Portanto, $G^{\prime}$ pode ser gerado por $(n-1)(n-2) / 2$ elementos.

O próximo passo é mostrar que $G^{\prime}$ tem expoente $n=[G: Z(G)]$. Isto é uma aplicação do homomorfismo Transfer, que definimos na 
Seção 1.7. Desenvolveremos um método para calcular o valor $\tau(g)$ do homomorfismo Transfer.

Por comodidade, denotamos $Z=Z(G)$. Considere a ação de $G$ em $G / Z$ por multiplicação à direita: para cada $g \in G$, temos uma permutação $\sigma_{g}: G / Z \rightarrow G / Z$ tal que $\sigma_{g}(Z x)=Z x g$, para qualquer $Z x \in G / Z$. Vamos escrever a permutação $\sigma_{g}$ na notação de ciclos disjuntos:

$$
\left(Z x_{1}, Z x_{1} g, \ldots, Z x_{1} g^{k_{1}-1}\right) \ldots\left(Z x_{s}, Z x_{s} g, \ldots, Z x_{s} g^{k_{s}-1}\right)
$$

onde $Z x_{1}, \ldots, Z x_{s} \in G / Z$ são representantes das diferentes $\langle g\rangle$-órbitas de $G / Z$ e, para $1 \leq i \leq s$, o inteiro positivo $k_{i}$ é o menor tal que $Z x_{i} g^{k_{i}}=Z x_{i}$. Observamos que $k_{1}+\cdots+k_{s}=n$.

Proposição 2.1.2. Seja $G$ um grupo tal que $[G: Z(G)]=n . O$ homomorfismo Transfer $\tau: G \rightarrow Z(G)$ é tal que $g \mapsto g^{n}$.

Demonstração. Continuamos com a notação acima. Observe que o conjunto $\left\{x_{i} g^{j} \mid 1 \leq i \leq s, 0 \leq j \leq k_{i}-1\right\}$ forma um transversal para $Z$ em $G$. Pela Proposição 1.7.1, podemos usar esse transversal para calcular $\tau(g)$. Como $Z x_{i} g^{k_{i}}=Z x_{i}$, para todo $1 \leq i \leq s$, temos que

$$
\tau(g)=\prod_{i=1}^{s}\left(x_{i}\right) g\left(x_{i} g\right)^{-1} \cdot\left(x_{i} g\right) g\left(x_{i} g^{2}\right)^{-1} \cdots\left(x_{i} g^{k_{i}-1}\right) g x_{i}^{-1}=\prod_{i=1}^{s} x_{i} g^{k_{i}} x_{i}^{-1} .
$$

Finalmente, note que $x_{i} g^{k_{i}} x_{i}^{-1} \in Z$, para $1 \leq i \leq s$. Logo, $g^{k_{i}} \in Z$. Assim, $x_{i} g^{k_{i}} x_{i}^{-1}=g^{k_{i}}$ e, portanto, $\tau(g)=g^{k_{1}} \cdots g^{k_{s}}=g^{k_{1}+\cdots+k_{s}}=g^{n}$, pois $k_{1}+\cdots+k_{s}=n$.

Corolário 2.1.3. Seja $G$ um grupo. Se $[G: Z(G)]=n$ então $x^{n}=1$, para todo $x \in G^{\prime}$. 
Demonstração. Pela Proposição 2.1.2, sabemos que o homomorfismo Transfer $\tau: G \rightarrow Z(G)$ é tal que $\tau(g)=g^{n}$. Pelo Teorema do Isomorfismo, $G / \operatorname{Ker}(\tau)$ é isomorfo a um subgrupo de $Z(G)$ e, portanto, $G / \operatorname{Ker}(\tau)$ é abeliano. Logo, $G^{\prime} \leq \operatorname{Ker}(\tau)$, pela Proposição 1.3.1. Assim, $x^{n}=1$, para todo $x \in G^{\prime}$.

Vamos agora apresentar o teorema clássico de Schur.

Teorema 2.1.4 (Schur). Seja $G$ um grupo. Se $[G: Z(G)]$ é finito então $G^{\prime}$ é finito. Além disso, $\left|G^{\prime}\right| \leq f([G: Z(G)])$, onde $f$ é uma função tal que $f(n)=n^{n\left(\begin{array}{c}n-1 \\ 2\end{array}\right)-n+2}$.

DemonstraçÃo. Seja $n=[G: Z(G)]$. Lembramos que, pelo Lema 2.1.1, $G^{\prime}$ pode ser gerado por $\left(\begin{array}{c}n-1 \\ 2\end{array}\right)$ elementos, e que $g^{n}=1$, para todo $g \in G^{\prime}$, pelo Corolário 2.1.3.

Vamos mostrar que o subgrupo normal $G^{\prime} \cap Z(G) \leq G^{\prime}$ também é finitamente gerado. Pelo segundo Teorema do Isomorfismo, temos que $\left[G^{\prime}: G^{\prime} \cap Z(G)\right]=\left[G^{\prime} Z(G): Z(G)\right] \leq[G: Z(G)]=n$. Sendo $G^{\prime}$ finitamente gerado, o Teorema 1.1.9 nos garante que $G^{\prime} \cap Z(G)$ pode ser gerado por no máximo $n\left(\begin{array}{c}n-1 \\ 2\end{array}\right)-n+1$ elementos.

Obviamente, $G^{\prime} \cap Z(G)$ é abeliano e tem expoente no máximo $n$. Assim, pelo Lema 1.1.2, temos que $\left|G^{\prime} \cap Z(G)\right| \leq n^{n\left(\begin{array}{c}n-1 \\ 2\end{array}\right)-n+1}$. Concluímos que $\left|G^{\prime}\right|=\left[G^{\prime}: G^{\prime} \cap Z(G)\right] \cdot\left|G^{\prime} \cap Z(G)\right| \leq n^{n\left(\begin{array}{c}n-1 \\ 2\end{array}\right)-n+2}$.

Como mostra o teorema a seguir, a cota do Teorema de Schur ainda pode ser melhorada usando técnicas elementares da Teoria de Grupos (o que não significa que a demonstração é trivial).

TeOrema 2.1.5. [26, p. 102] Seja $G$ um grupo tal que $[G: Z(G)] e ́$ finito. Então $\left|G^{\prime}\right| \leq n^{n\left\lfloor\log _{p} n\right\rfloor-n+2}$, onde $n=[G: Z(G)]$ e p é o menor primo divisor de $n$. 
Demonstração. Como $G / Z(G)$ tem ordem $n$, pela Proposição 1.1.4, $G / Z(G)$ pode ser gerado por $r \leq\left\lfloor\log _{p} n\right\rfloor$ elementos. Sejam $g_{1}, \ldots, g_{r} \in G$ tais que $G / Z(G)=\left\langle g_{1} Z(G), \ldots, g_{r} Z(G)\right\rangle$ e defina $U=$ $\left\langle g_{1}, \ldots, g_{r}\right\rangle$. Como $[U: U \cap Z(G)]=[U Z(G): Z(G)] \leq n$, segue do Teorema 1.1.9 que $U \cap Z(G)$ pode ser gerado por uma quantidade de elementos menor ou igual a $n r-n+1$.

Note que $G=U Z(G)$ e, assim, $G^{\prime} \leq U^{\prime} \leq U$. Portanto, temos que $G^{\prime} \cap Z(G) \leq U \cap Z(G)$. Pela Proposição 1.1.7, $G^{\prime} \cap Z(G)$ também pode ser gerado por uma quantidade de elementos menor ou igual a $n r-n+1$, pois $U \cap Z(G)$ é abeliano.

Segue do Lema 1.1.2 que $\left|G^{\prime} \cap Z(G)\right| \leq n^{n r-n+1}$, pois $\left(G^{\prime}\right)^{n}=1$. Como o índice de $G^{\prime} \cap Z(G)$ em $G^{\prime}$ é menor ou igual a $n$, concluímos que $\left|G^{\prime}\right| \leq n n^{n r-n+1}=n^{n r-n+2}$. Lembrando que $r \leq\left\lfloor\log _{p} n\right\rfloor$, temos que $\left|G^{\prime}\right| \leq n^{n\left\lfloor\log _{p} n\right\rfloor-n+2}$.

A melhor cota possível para um qualquer grupo $G$ foi deduzida por Wiegold em [30], usando a Teoria do Multiplicador de Schur, a qual está fora do escopo dessa dissertação.

Teorema 2.1.6. [30] Seja $G$ um grupo tal que $[G: Z(G)]$ é finito. Então $\left|G^{\prime}\right| \leq n^{\frac{1}{2}\left(\log _{p} n-1\right)}$, onde $n=[G: Z(G)]$ e p é o menor primo divisor de $n$.

\subsection{Recíprocas do Teorema de Schur}

Como vimos na Seção 1.5, para todo primo $p$, existe um $p$-grupo infinito $E$ que é extra especial, isto é, $E^{\prime}=Z(E)$ tem ordem $p$ e $G / Z(G)$ é abeliano elementar infinito. Isto mostra que a recíproca do Teorema de Schur não vale em geral. Explicitamos a seguir um outro 
exemplo para ver que a recíproca do Teorema de Schur não vale em geral.

Exemplo 2.2.1. [23, Example 2.2] Sejam $p$ um primo e $N$ e $A$ dois $p$-grupos abelianos elementares de bases enumeráveis $\left\{x_{i}\right\}_{i \geq 0}$ e $\left\{a_{j}\right\}_{j \geq 1}$, respectivamente. Definimos uma ação de $A$ em $N$ tal que, para todo $i \geq 1$, tem-se $x_{i}^{a_{i}}=x_{i} x_{0}$ e, para $i \neq j$, tem-se $x_{i}^{a_{j}}=x_{i}$. No produto semidireto $G=N \rtimes A$, é fácil verificar que $Z(G)=G^{\prime}=\left\langle x_{0}\right\rangle$, pois $\left[x_{i}, a_{i}\right]=x_{0}$, para todo $i \geq 1$, e $\left[x_{i}, a_{j}\right]=1$, para $i \neq j$. Assim, $G^{\prime}$ é finito de ordem $p$, mas $[G: Z(G)]$ é infinito.

O que discutimos nesta seção são recíprocas do Teorema de Schur obtidas impondo condições a mais sobre o grupo $G$.

Por exemplo, para os grupos finitamente gerados vale a recíproca do Teorema de Schur, como mostra a proposição a seguir devida a B. H. Neumann.

ProposiçÃo 2.2.2. [22, Corollary 5.41] Seja $G$ um grupo m-gerado. Se $G^{\prime}$ é finito então $[G: Z(G)] \leq\left|G^{\prime}\right|^{m}$.

Demonstração. Segue imediatamente da Proposição 1.6.6 que $[G: Z(G)]=\left[G: C_{G}(G)\right] \leq\left|G^{\prime}\right|^{m}$.

Corolário 2.2.3. Seja $G$ um grupo finitamente gerado. Se $G^{\prime}$ é finito então $Z(G)$ é finitamente gerado.

Demonstração. Pela Proposição 2.2.2, $[G: Z(G)]$ é finito, então, do Teorema 1.1.9, $Z(G)$ é finitamente gerado.

Em [23] Niroomand mostrou que a recíproca do Teorema de Schur também vale para grupos cujo quociente central é finitamente gerado. 
Ou seja, a Proposição 2.2.2 ainda é válida sob a hipótese mais fraca de que $G / Z(G)$ seja finitamente gerado.

Teorema 2.2.4. [23, Main Theorem] Seja G um grupo tal que $G / Z(G)$ é m-gerado. Se $G^{\prime}$ é finito então $[G: Z(G)] \leq\left|G^{\prime}\right|^{m}$.

DemonstraçÃo. Seja $G / Z(G)=\left\langle g_{1} Z(G), \ldots, g_{m} Z(G)\right\rangle$ e defina

$$
\begin{aligned}
\varphi: G / Z(G) & \longrightarrow G^{\prime} \times \cdots \times G^{\prime} \\
x Z(G) & \longmapsto\left(\left[x, g_{1}\right], \ldots,\left[x, g_{m}\right]\right)
\end{aligned}
$$

Se $x Z(G)=y Z(G), \operatorname{com} x, y \in G$, então $y=x z$, para algum $z \in Z(G)$. Pela fórmula (1.2.1), $\left[y, g_{i}\right]=\left[x z, g_{i}\right]=\left[x, g_{i}\right]$, para todo $1 \leq i \leq m$. Logo $\varphi(y Z(G))=\varphi(x Z(G))$ e, portanto, $\varphi$ está bem definida.

Concluímos mostrando que $\varphi$ é injetiva. Suponha que $\varphi(x Z(G))=$ $\varphi(y Z(G)), \operatorname{com} x, y \in G$. Então, para todo $1 \leq i \leq m$, temos $\left[y, g_{i}\right]=$ $\left[x, g_{i}\right]$. Das identidades (1.2.1) e (1.2.8), temos que

$$
\begin{aligned}
{\left[y x^{-1}, g_{i}\right] } & =\left[y, g_{i}\right]^{\left(x^{-1}\right)}\left[x^{-1}, g_{i}\right]=\left[y, g_{i}\right]^{\left(x^{-1}\right)}\left[g_{i}, x\right]^{\left(x^{-1}\right)} \\
& =\left(\left[y, g_{i}\right]\left[g_{i}, x\right]\right)^{\left(x^{-1}\right)}=\left(\left[y, g_{i}\right]\left[x, g_{i}\right]^{-1}\right)^{\left(x^{-1}\right)}=1 .
\end{aligned}
$$

Ou seja, $y x^{-1} \in C_{G}\left(g_{i}\right)$, para $1 \leq i \leq m$. Como $G=\left\langle g_{1}, \ldots, g_{m}, Z(G)\right\rangle$, segue que $y x^{-1} \in Z(G)$ e, portanto, $y Z(G)=x Z(G)$. Isto mostra que $\varphi$ é injetiva. Assim, $[G: Z(G)] \leq\left|G^{\prime}\right|^{m}$.

Observe que, para obter uma cota mais apurada, podemos aplicar o Teorema 2.2 .4 com $m=d(G / Z(G))$, onde $d(G / Z(G))$ indica o número mínimo de geradores para $G / Z(G)$. De fato, assim é enunciado o resultado em $[\mathbf{2 3}]$. 


\subsection{Teorema de Baer}

O Teorema de Schur diz que se $[G: Z(G)]$ é finito então $G^{\prime}$ é finito. O Teorema de Baer generaliza esse resultado para outros termos das séries centrais ascendente e descendente, mostrando que se $\left[G: Z_{i}(G)\right]$ é finito, para algum $i \geq 0$, então $\gamma_{i+1}(G)$ é finito.

Para provar o Teorema de Baer precisamos do resultado a seguir, cuja demonstração (sem a versão quantitativa) está em [25, 14.5.2].

TeOrema 2.3.1. Sejam $M \leq H$ e $N \leq K$ subgrupos normais de um grupo $G$ tais que $[N, H]=1=[M, K]$. Se $[H: M]=m e[K: N]=n$ são finitos então $[H, K] \leq m^{n}(m n)^{\log _{p}(m n)-1}$, onde $p$ é o menor primo que divide $m n$.

DemonstraÇÃo. Seja $I=H \cap K$. Observe que $C_{K}(I)$ é normal em $K$, pois $I$ é normal em $K$. Pela Proposição 1.6.1, a aplicação $\alpha$, definida abaixo, é um homomorfismo.

$$
\begin{aligned}
\alpha: K / C_{K}(I) & \longrightarrow \operatorname{Aut}(I) \\
k C_{K}(I) & \longmapsto\left(x \mapsto x^{k}\right)
\end{aligned}
$$

Consideramos $P=I \rtimes_{\alpha}\left(K / C_{K}(I)\right)$. Pela Proposição 1.6.2, temos que $\left[I, K / C_{K}(I)\right]=[I, K]$. Então $\left[M \cap I, K / C_{K}(I)\right]=[M \cap I, K]=1$. Além disso, $[M \cap I, I]=1$. Como $P$ é gerado por $K / C_{K}(I)$ e $I$, segue que $[M \cap I, P]=1$, ou seja, $M \cap I \leq Z(P)$. Portanto,

$$
[P: Z(P)] \text { divide }[P: M \cap I]=[P: I][I: M \cap I]
$$

Observe que $[P: I]=\left|K / C_{K}(I)\right|$ divide $[K: N]$, pois $N \leq C_{K}(I)$, e observe que $[I: M \cap I]=[M I: M]$ divide $[H: M]$. Assim, da relação 
(2.3.1) concluímos que

$$
[P: Z(P)] \text { divide }[H: M][K: N]=m n
$$

Sejam $p$ o menor primo que divide $m n$ e $q$ o menor primo que divide $[P: Z(P)]$. Segue do Teorema 2.1.6 que

$$
\left|P^{\prime}\right| \leq[P: Z(P)]^{\frac{1}{2}\left(\log _{q}[P: Z(P)]-1\right)} \leq(m n)^{\frac{1}{2}\left(\log _{q}(m n)-1\right)}
$$

pois $[P: Z(P)] \leq m n$, pela relação (2.3.2). Da relação (2.3.2) também segue que $p \leq q$ e, portanto, $\log _{q}(m n) \leq \log _{p}(m n)$. Então, da relação (2.3.3) segue que

$$
\left|P^{\prime}\right| \leq(m n)^{\frac{1}{2}\left(\log _{p}(m n)-1\right)}
$$

Pela Proposição 1.6.2, temos que $[I, K]=\left[I, K / C_{K}(I)\right] \leq P^{\prime}$. Portanto, da desigualdade (2.3.4) segue que

$$
|[I, K]| \leq(m n)^{\frac{1}{2}\left(\log _{p}(m n)-1\right)}
$$

Para $[I, H]$, um argumento simétrico nos garante que

$$
|[I, H]| \leq(m n)^{\frac{1}{2}\left(\log _{p}(m n)-1\right)} .
$$

Das desigualdades (2.3.5) e (2.3.6) concluímos que

$$
|[H, I][K, I]| \leq(m n)^{\log _{p}(m n)-1}
$$

Agora vamos mostrar que $[H, K] /[H, I][K, I]$ é finito. Como já mostramos que $[H, I][K, I]$ é finito, podemos supor, sem perda de generalidade, que

$$
[H, I][K, I]=1 \text {. }
$$


Como $H$ e $K$ são normais em $G$, temos que $[H, K] \leq I$. Então, segue do Lema 1.3.5 que $[H, H, K] \leq[H, K, H][H, K, H] \leq[I, H]=1$. Analogamente $[K, K, H]=1$. Por hipótese, $[M, K]=1=[N, H]$. Portanto,

$$
\left[H^{\prime} M, K\right]=1=\left[K^{\prime} N, H\right]
$$

e, assim, podemos definir a aplicação

$$
\begin{aligned}
\varphi: H / H^{\prime} M \times K / K^{\prime} N & \longrightarrow[H, K] . \\
\left(h H^{\prime} M, k K^{\prime} N\right) & \longmapsto[h, k]
\end{aligned}
$$

De fato, se $\left(h_{2} H^{\prime} M, k_{2} K^{\prime} N\right)=\left(h H^{\prime} M, k K^{\prime} N\right)$, com $h, h_{2} \in H$ e $k, k_{2} \in K$, então $h_{2}=h x, \operatorname{com} x \in H^{\prime} M$, e $k_{2}=k y$, com $y \in K^{\prime} N$. Logo, $\left[h_{2}, k_{2}\right]=\left[h x, k_{2}\right]=\left[h, k_{2}\right]^{x}\left[x, k_{2}\right]=\left[h, k_{2}\right]$, pois $\left[H^{\prime} M, K\right]=1$. Assim, $\left[h_{2}, k_{2}\right]=[h, k y]=[h, y][h, k]^{y}=[h, k]$, pois $\left[K^{\prime} N, H\right]=1$.

Vamos mostrar agora que da hipótese (2.3.8) segue que $\varphi$ é bilinear. Sejam $h_{1}, h_{2} \in H$ e $k_{1}, k_{2} \in K$. Da identidade (1.2.5), segue que $\left[h_{1} h_{2}, k_{1}\right]=\left[h_{1}, k_{1}\right]\left[h_{1}, k_{1}, h_{2}\right]\left[h_{2}, k_{1}\right]=\left[h_{1}, k_{1}\right]\left[h_{2}, k_{1}\right]$, pois $[I, H]=1$ pela hipótese (2.3.8). Analogamente, da identidade (1.2.6), segue que $\left[h_{1}, k_{1} k_{2}\right]=\left[h_{1}, k_{2}\right]\left[h_{1}, k_{1}\right]\left[h_{1}, k_{1}, k_{2}\right]=\left[h_{1}, k_{1}\right]\left[h_{1}, k_{2}\right]$, pois $[I, K]=1$ pela hipótese (2.3.8). Isto mostra que $\varphi$ é bilinear.

Observamos que $H / H^{\prime} M$ e $K / K^{\prime} N$ são abelianos, pois $H^{\prime} \leq H^{\prime} M$ e $K^{\prime} \leq K^{\prime} N$. Logo, pela definição de produto tensorial de grupos abelianos, existe um homomorfismo $\rho: H / H^{\prime} M \otimes K / K^{\prime} N \longrightarrow[H, K]$ tal que $\rho\left(h H^{\prime} M \otimes k K^{\prime} N\right)=\varphi\left(h H^{\prime} M, k K^{\prime} N\right)=[h, k]$, quaisquer que sejam $h \in H$ e $k \in K$. Note que os geradores de $[H, K]$ estão contidos na imagem de $\rho$ e, portanto, $\rho$ é sobrejetivo. Assim, concluímos que

$$
|[H, K]| \leq\left|H / H^{\prime} M \otimes K / K^{\prime} N\right| \leq\left|H / H^{\prime} M\right|^{\left|K / K^{\prime} N\right|}
$$


pela Proposição 1.8.3. Notando que $M \leq H^{\prime} M$ e que $N \leq K^{\prime} N$, vemos que $\left|H / H^{\prime} M\right| \leq[H: M]=m$ e que $\left|K / K^{\prime} N\right| \leq[K: N]=n$. Portanto,

$$
|[H, K]| \leq m^{n}
$$

Lembre-se que assumimos (2.3.8) e trabalhamos até agora no quociente $[H, K] /[I, H][I, K]$. Assim, no caso geral, a desigualdade (2.3.9) significa que $[H, K] /[I, H][I, K]$ tem ordem menor ou igual a $m^{n}$. Disso e da relação (2.3.7) segue que

$$
\begin{aligned}
|[H, K]| & =[[H, K]:[I, H][I, K]] \cdot[I, H][I, K] \\
& \leq m^{n} \cdot(m n)^{\log _{p}(m n)-1} .
\end{aligned}
$$

Agora vamos ver a demonstração do Teorema de Baer.

Teorema 2.3.2 (Baer). Seja $G$ um grupo. Se, para algum $i \geq 0$, $\left[G: Z_{i}(G)\right]$ é finito então $\gamma_{i+1}(G)$ é finito. Além disso, existe uma função $f$ tal que $\left|\gamma_{i+1}(G)\right| \leq f\left(\left[G: Z_{i}(G)\right]\right)$.

Demonstração. Por simplicidade denotamos $n=\left[G: Z_{i}(G)\right]$. Usamos indução sobre $i$. O caso $i=0$ é óbvio e o caso $i=1$ é precisamente o Teorema de Schur. Sendo assim, considere $i>1$. Observe que $\left[G / Z(G): Z_{i-1}(G / Z(G))\right]=\left[G: Z_{i}(G)\right]$, que é finito por hipótese. Então, por indução sobre $i, \gamma_{i}(G / Z(G))$ é finito com ordem limitada em função de $n$. Lembrando que $\gamma_{i}(G / Z(G))=\gamma_{i}(G) Z(G) / Z(G)$, segue que $\gamma_{i}(G) Z(G) / Z(G)$ é finito com ordem limitada em função de $n$.

Sejam $M=Z(G), H=\gamma_{i}(G) Z(G), N=Z_{i}(G)$ e $K=G$. Pelo que observamos acima, $\left[\gamma_{i}(G) Z(G): Z(G)\right]$ é limitado em função de $n$ e, por 
hipótese, $\left[G: Z_{i}(G)\right]=n$. Note ainda, que $[Z(G), G]=1$ e que, pela Proposição 1.3.6, temos que $\left[Z_{i}(G), \gamma_{i}(G) Z(G)\right]=\left[Z_{i}(G), \gamma_{i}(G)\right]=1$. Segue do Teorema 2.3 .1 que $\left[\gamma_{i}(G) Z(G), G\right]=\left[\gamma_{i}(G), G\right]=\gamma_{i+1}(G)$ é finito com ordem limitada em função de $n$.

O Teorema 2.3.2 é conhecido como Teorema de Baer, mas o resultado provado por Baer em [2] possui uma formulação muito mais geral:

Teorema 2.3.3. [2, $§ 6$ Theorem 4] Seja $N$ um subgrupo normal de um grupo $G$ tal que $[G: N]$ é finito. Então $\left[\gamma_{n+1}(G):\left[N,_{n} G\right]\right]$ é finito, para todo $n \geq 0$, e todo número primo que divide $\left[\gamma_{n+1}(G):\left[N,_{n} G\right]\right]$ também divide $[G: N]$.

Observe que se $\left[G: Z_{i}(G)\right]$ é finito, tomando $N=Z_{i}(G)$ e $n=i$, obtemos o Teorema 2.3.2, pois $\left[Z_{i}(G),{ }_{i} G\right]=1$.

\subsection{Recíprocas do Teorema de Baer}

A recíproca do Teorema de Baer não vale em geral. De fato, para cada $i \geq 1$, P. Hall fornece um exemplo de um p-grupo $G$ com $\gamma_{i+1}(G)$ finito e $\left[G: Z_{i}(G)\right]$ infinito. Nos restringimos a dar o enunciado desses exemplos. Para as justificativas adequadas das suas propriedades veja as páginas 614-616 do artigo [11] de P. Hall.

Para discutir os exemplos de Hall precisaremos do conceito de produto central. Sejam $Z$ um grupo abeliano fixado e $\left\{G_{i} \mid i \in I\right\}$ uma família de grupos tais que $Z\left(G_{i}\right)$ é isomorfo a $Z$, para todo $i \in I$. Dizemos que o grupo $G$ é o produto central de $\left\{G_{i} \mid i \in I\right\}$ se, para cada $i \in I$, existe um subgrupo $H_{i} \leq G$ isomorfo a $G_{i}$ e as seguintes condições são satisfeitas: $G=\left\langle\bigcup_{i \in I} H_{i}\right\rangle, Z(G) \cong Z$ e, para todo $i \neq j,\left[H_{i}, H_{j}\right]=1$ e $H_{i} \cap H_{j}=Z(G)$. Quando $|I|=2$ o produto 
central é isomorfo à seguinte construção: $G=(H \times K) / N$, onde $N=\left\langle\left(z^{-1}, \alpha(z)\right) \mid z \in Z(H)\right\rangle$ e $\alpha: Z(H) \rightarrow Z(K)$ é um isomorfismo. O grupo $G$ é chamado de produto central de $H$ e $K$. Note que $H$ é isomorfo a $(H \times 1) / N$ e que $K$ é isomorfo a $(1 \times K) / N$. Assim, se identificarmos $H \operatorname{com}(H \times 1) / N$ e $K \operatorname{com}(1 \times K) / N$, é possível mostrar que $G=H K, Z(G) \cong Z(H),[H, K]=1$ e $H \cap K=Z(G)$. Para mais detalhes sobre o produto central veja [8, pp. 27-29].

Seja $p$ um primo. Se $p=2$, considere $E=E(p)$ como sendo o produto central de uma quantidade infinita enumerável de cópias do grupo dos quatérnios de ordem 8 . Se $p>2$, seja $E=E(p)$ o produto central de uma quantidade infinita enumerável de cópias do grupo não abeliano de ordem $p^{3}$ e expoente $p$. Segundo Hall, o grupo $E$ é tal que $E^{\prime}=Z(E)$ é cíclico de ordem $p$ e $E / E^{\prime}$ é um $p$-grupo abeliano elementar de ordem infinita. Assim, $E$ é um p-grupo extra especial infinito, conforme a Definição 1.5.1. Defina

$$
G=G(p)=E(p) \curlywedge C_{p}
$$

o produto entrelaçado de $E(p)$ por $C_{p}=\left\langle c \mid c^{p}=1\right\rangle$. Isto é, o grupo $G$ é definido como o produto semidireto de $E(p) \times \stackrel{p}{\cdots} \times E(p)$ por $C_{p}$, cuja ação é tal que, para todo $\left(u_{1}, \ldots, u_{p}\right) \in E(p) \times \cdots \times E(p)$, tem-se $\left(u_{1}, \ldots, u_{p-1}, u_{p}\right)^{c}=\left(u_{2}, \ldots, u_{p}, u_{1}\right)$. Hall provou que $G$ é um $p$-grupo de classe $2 p$ e com as seguintes propriedades

$$
\begin{gathered}
Z_{j}(G)=\gamma_{2 p-j+1}(G), \text { para todo } 0 \leq j \leq 2 p ; \\
{\left[Z_{j}(G): Z_{j-1}(G)\right]=p \text {, para todo } 1 \leq j \leq p ;} \\
{\left[Z_{j}(G): Z_{j-1}(G)\right] \text { é infinito, para todo } p+1 \leq j \leq 2 p .}
\end{gathered}
$$

Agora, dado $i>1$, seja $p$ um primo tal que $p \leq i<2 p$ e considere o grupo $G=G(p)$. Pelas propriedades acima, $\gamma_{i+1}(G)=Z_{2 p-i}(G)$ 
é finito, enquanto $\left[Z_{i+1}(G): Z_{i}(G)\right]$ é infinito e, consequentemente, $\left[G: Z_{i}(G)\right]$ também é infinito.

No entanto, assim como vimos para o Teorema de Schur, a recíproca do Teorema de Baer vale para os grupos finitamente gerados. A demonstração que apresentamos é devida a Hekster e se baseia na seguinte proposição.

ProposiçÃo 2.4.1. [16, p. 68] Seja $G$ um grupo m-gerado. Se, para $i \geq 1, \gamma_{i+1}(G)$ é finito então $\left[\gamma_{i}(G): \gamma_{i}(G) \cap Z(G)\right] \leq\left|\gamma_{i+1}(G)\right|^{m}$.

Demonstração. Observamos que o caso $i=1$ é o conteúdo da Proposição 2.2.2. Como $\gamma_{i+1}(G)=\left[\gamma_{i}(G), G\right]$ é finito, aplicando a Proposição 1.6.6, temos que $\left[\gamma_{i}(G): C_{\gamma_{i}(G)}(G)\right] \leq\left|\gamma_{i+1}(G)\right|^{m}$. Notando que $C_{\gamma_{i}(G)}(G)=\gamma_{i}(G) \cap Z(G)$, concluímos a demonstração.

TeOrema 2.4.2. [16, Theorem 2.10] Seja $G$ um grupo m-gerado. Se, para algum $i \geq 0, \gamma_{i+1}(G)$ é finito então $\left[G: Z_{i}(G)\right] \leq\left|\gamma_{i+1}(G)\right|^{m^{i}}$.

Demonstração. Argumentaremos por indução sobre $i$. O caso $i=0$ é óbvio. Agora, seja $i \geq 1$. Aplicando a Proposição 2.4.1, temos que $\left|\gamma_{i}(G / Z(G))\right|=\left[\gamma_{i}(G): \gamma_{i}(G) \cap Z(G)\right] \leq\left|\gamma_{i+1}(G)\right|^{m}$. Note que $G / Z(G)$ também é $m$-gerado, então, da hipótese de indução aplicada a $G / Z(G)$, temos $\left[G / Z(G): Z_{i-1}(G / Z(G))\right] \leq\left|\gamma_{i}(G / Z(G))\right|^{m^{i-1}} \leq$ $\left(\left|\gamma_{i+1}(G)\right|^{m}\right)^{m^{i-1}}=\left|\gamma_{i+1}(G)\right|^{m^{i}}$. Concluímos a demonstração observando que $\left[G: Z_{i}(G)\right]=\left[G / Z(G): Z_{i-1}(G / Z(G))\right]$.

Corolário 2.4.3. Seja $G$ um grupo finitamente gerado. Se $\gamma_{i+1}(G)$ é finito, para algum $i \geq 0$, então $Z_{i+j}(G)$ é finitamente gerado, para todo $j \geq 0$. 
Demonstração. Do Teorema 2.4 .2 segue que $\left[G: Z_{i+j}(G)\right]$ é finito, para todo $j \geq 0$, já que $\left[G: Z_{i+j}(G)\right] \leq\left[G: Z_{i}(G)\right]$. O resultado segue aplicando o Teorema 1.1.9.

Em 2014, generalizando ao mesmo tempo o Teorema 2.2.4 de Niroomand e o Teorema 2.4.2, Hatamian, Hassanzadeh e Kayvanfar [14] mostraram que a recíproca do Teorema de Baer vale para grupos $G$ cujo quociente $G / Z_{i}(G)$ é finitamente gerado, para algum $i \geq 0$. Mais precisamente, temos o seguinte resultado.

TeOrema 2.4.4. Seja $G$ um grupo tal que $G / Z_{i}(G)$ é m-gerado, para algum $i \geq 0$. Se $\gamma_{i+1}(G)$ é finito então $\left[G: Z_{i}(G)\right] \leq\left|\gamma_{i+1}(G)\right|^{m^{i}}$.

DemonstraÇÃo. Seja $G / Z_{i}(G)=\left\langle g_{1} Z_{i}(G), \ldots, g_{m} Z_{i}(G)\right\rangle$, com $g_{1}, \ldots, g_{m} \in G$, e defina $U=\left\langle g_{1}, \ldots, g_{m}\right\rangle$. Primeiro mostramos que

$$
\gamma_{i+1}(G)=\gamma_{i+1}(U) \quad \text { e } \quad\left[G: Z_{i}(G)\right]=\left[U: Z_{i}(U)\right]
$$

Sejam $x_{1}, \ldots, x_{i+1} \in G$. Note que $G=U Z_{i}(G)$, então, para todo $1 \leq k \leq i+1$, existem $u_{k} \in U$ e $z_{k} \in Z_{i}(G)$ tais que $x_{k}=u_{k} z_{k}$. Assim, $\left[x_{1}, \ldots, x_{i+1}\right]=\left[u_{1} z_{1}, \ldots, u_{i+1} z_{i+1}\right]=\left[u_{1}, \ldots, u_{i+1}\right]$, pela Proposição 1.3.7. Isto mostra que $\left\{\left[x_{1}, \ldots, x_{i+1}\right] \mid x_{k} \in G\right\} \subseteq \gamma_{i+1}(U)$. Segue da Proposição 1.3.9 que $\gamma_{i+1}(G) \subseteq \gamma_{i+1}(U)$. A inclusão reversa é óbvia e, portanto, $\gamma_{i+1}(G)=\gamma_{i+1}(U)$, demonstrando a primeira identidade em (2.4.1).

Agora, $\left[G: Z_{i}(G)\right]=\left[U Z_{i}(G): Z_{i}(G)\right]=\left[U: U \cap Z_{i}(G)\right]$. Assim, para demonstrar a segunda identidade em (2.4.1), basta mostrar que $Z_{i}(U)=U \cap Z_{i}(G)$. Dados $u \in Z_{i}(U)$ e $x_{1}, \ldots, x_{i} \in G$, para todo $1 \leq j \leq i$, existe $u_{j} \in U$ e $z_{j} \in Z_{i}(G)$ tal que $x_{j}=u_{j} z_{j}$. 
Então $\left[u, x_{1}, \ldots, x_{i}\right]=\left[u, u_{1} z_{1}, \ldots, u_{i} z_{i}\right]=\left[u, u_{1}, \ldots, u_{i}\right]$, pela Proposição 1.3.7. Como $u \in Z_{i}(U)$, temos que $\left[u, u_{1}, \ldots, u_{i}\right]=1$, ou seja, $\left[u, x_{1}, \ldots, x_{i}\right]=1$. Isto mostra que $u \in U \cap Z_{i}(G)$ e, sendo $u$ um elemento arbitrário de $Z_{i}(U)$, segue que $Z_{i}(U) \leq U \cap Z_{i}(G)$. Por outro lado, é claro que $U \cap Z_{i}(G) \leq Z_{i}(U)$. Portanto, $Z_{i}(U)=U \cap Z_{i}(G)$, como queríamos.

Como $U$ é $m$-gerado e $\gamma_{i+1}(U)=\gamma_{i+1}(G)$ é finito, aplicando o Teorema 2.4.2 ao grupo $U$, temos que $\left[U: Z_{i}(U)\right] \leq\left|\gamma_{i+1}(U)\right|^{m^{i}}$. Concluímos usando (2.4.1) que $\left[G: Z_{i}(G)\right] \leq\left|\gamma_{i+1}(G)\right|^{m^{i}}$.

Para obter uma cota mais precisa, podemos aplicar o Teorema 2.4.4 com $m=d\left(G / Z_{i}(G)\right)$, onde $d\left(G / Z_{i}(G)\right)$ indica o número mínimo de geradores do grupo $G / Z_{i}(G)$, e de fato assim o resultado está enunciado em $[\mathbf{1 4}]$.

Lembramos que um grupo $G$ é dito residualmente finito se, para todo $1 \neq g \in G$, existe um subgrupo normal $N=N(g)$ tal que $g \notin N$ e $G / N$ é finito. O teorema abaixo estabelece a recíproca do Teorema de Baer para os grupos residualmente finitos. A demonstração que apresentamos foi dada por Macdonald em [20].

TeOrema 2.4.5. Seja $G$ um grupo residualmente finito. Se, para algum $i \geq 0, \gamma_{i+1}(G)$ é finito então $\left[G: Z_{i}(G)\right]$ é finito.

Demonstração. Afirmamos que existe um subgrupo normal $N$ de índice finito em $G$ tal que $\gamma_{i+1}(G) \cap N=1$. De fato, para cada $x \in \gamma_{i+1}(G) \backslash\{1\}$, existe um subgrupo normal $N(x)$ tal que $x \notin N(x)$ e $G / N(x)$ é finito. Seja

$$
N=\bigcap_{x \in \gamma_{i+1}(G) \backslash\{1\}} N(x) .
$$


Note que $N$ é um subgrupo normal de $G$, pois é uma interseção de subgrupos normais. Como $\gamma_{i+1}(G)$ é finito, temos que $G / N$ é finito, pela Proposição 1.0.2. Agora, se $x \in \gamma_{i+1}(G) \backslash\{1\}$ então $x \notin N(x)$ e, portanto, $x \notin N$. Assim, $\gamma_{i+1}(G) \cap N=1$.

Para concluir, é suficiente mostrar que $N \leq Z_{i}(G)$. Como $\left[N,_{i} G\right] \leq$ $\gamma_{i+1}(G) \cap N$, temos que $\left[N,{ }_{i} G\right]=1$ e, assim, pela Proposição 1.3.3, $N \leq Z_{i}(G)$.

Concluímos esta seção com uma recíproca do Teorema de Baer para grupos que possuem um subgrupo abeliano de índice finito. Precisaremos de um lema que foi inspirado pelo Lema 2.2 de [17].

Lema 2.4.6. Seja $G$ um grupo que contém um subgrupo abeliano $A$ de índice finito. Se, para algum inteiro $i \geq 1, \gamma_{i+1}(G)$ é finito então $\left|\gamma_{i}(G / Z(G))\right| \leq[G: A]^{1+\log _{2}\left|\gamma_{i+1}(G)\right|}$.

Demonstração. Se $A=G$, o resultado é óbvio. Assuma que $A$ é um subgrupo próprio de $G$. Assim, pela Proposição 1.1.3, existem um inteiro $1 \leq m \leq \log _{2}[G: A]$ e elementos $x_{1}, \ldots, x_{m} \in G$ tais que $G=\langle X, A\rangle$, onde $X=\left\{x_{1}, \ldots, x_{m}\right\}$.

Lembramos que $C_{\gamma_{i}(G)}(G)=\gamma_{i}(G) \cap Z(G)$ e, portanto, temos que $\left|\gamma_{i}(G / Z(G))\right|=\left[\gamma_{i}(G): \gamma_{i}(G) \cap Z(G)\right]=\left[\gamma_{i}(G): C_{\gamma_{i}(G)}(G)\right]$. Como $A$ é abeliano, segue que $A \cap C_{\gamma_{i}(G)}(X) \leq C_{\gamma_{i}(G)}(G)$ e, assim, é suficiente mostrar que $\left[\gamma_{i}(G): A \cap C_{\gamma_{i}(G)}(X)\right]$ é finito. Agora, $\left[\gamma_{i}(G): A \cap \gamma_{i}(G)\right] \leq$ $[G: A]$, que é finito, e só falta mostrar que $\left[A \cap \gamma_{i}(G): A \cap C_{\gamma_{i}(G)}(X)\right]$ 
é finito. Observe que, pela Proposição 1.0.1,

$$
\begin{aligned}
{\left[A \cap \gamma_{i}(G): A \cap C_{\gamma_{i}(G)}(X)\right] } & =\left[A \cap \gamma_{i}(G):\left(A \cap \gamma_{i}(G)\right) \cap C_{\gamma_{i}(G)}(X)\right] \\
& =\left|\left\{x C_{\gamma_{i}(G)}(X) \mid x \in A \cap \gamma_{i}(G)\right\}\right| \\
& \leq\left[\gamma_{i}(G): C_{\gamma_{i}(G)}(X)\right] .
\end{aligned}
$$

Agora, notando que $C_{\gamma_{i}(G)}(X)=\bigcap_{j=1}^{m} C_{\gamma_{i}(G)}\left(x_{j}\right)$, concluímos que $\left[\gamma_{i}(G): C_{\gamma_{i}(G)}(X)\right] \leq \prod_{j=1}^{m}\left[\gamma_{i}(G): C_{\gamma_{i}(G)}\left(x_{j}\right)\right]$, pela Proposição 1.0.2. Finalmente, para cada $x \in G$, temos que $\left[\gamma_{i}(G): C_{\gamma_{i}(G)}(x)\right]=\left|x^{\gamma_{i}(G)}\right|$. Afirmamos que $\left|x^{\gamma_{i}(G)}\right| \leq\left|\gamma_{i+1}(G) x\right|=\left|\gamma_{i+1}(G)\right|$, para todo $x \in G$. Para ver isso note que $x^{g} x^{-1}=\left[g, x^{-1}\right] \in \gamma_{i+1}(G)$, para todo $g \in \gamma_{i}(G)$ e todo $x \in G$. Então $x^{g} \in \gamma_{i+1}(G) x$. Segue que $x^{\gamma_{i}(G)} \subseteq \gamma_{i+1}(G) x$, e isso prova a afirmação. Concluímos que $\left[\gamma_{i}(G): C_{\gamma_{i}(G)}(X)\right] \leq\left|\gamma_{i+1}(G)\right|^{m} \leq$ $\left|\gamma_{i+1}(G)\right|^{\log _{2}[G: A]}=[G: A]^{\log _{2}\left|\gamma_{i+1}(G)\right|}$, onde usamos a propriedade de que $a^{\log _{2} b}=b^{\log _{2} a}$.

Terminamos a demonstração retomando o que estabelecemos acima:

$$
\begin{aligned}
{\left[\gamma_{i}(G): C_{\gamma_{i}(G)}(G)\right] } & \leq\left[\gamma_{i}(G): A \cap \gamma_{i}(G)\right] \cdot\left[A \cap \gamma_{i}(G): A \cap C_{\gamma_{i}(G)}(X)\right] \\
& \leq[G: A] \cdot\left[\gamma_{i}(G): C_{\gamma_{i}(G)}(X)\right] \\
& \leq[G: A] \cdot[G: A]^{\log _{2}\left|\gamma_{i+1}(G)\right|}
\end{aligned}
$$

Agora podemos provar a recíproca do Teorema de Baer para grupos que contêm um subgrupo abeliano de índice finito. Mais precisamente temos o seguinte resultado.

TeOrema 2.4.7. Seja G um grupo que contém um subgrupo abeliano $A$ de índice finito $n$. Se, para algum $i \geq 1, \gamma_{i+1}(G)$ é finito 
então $\left[G: Z_{i}(G)\right] \leq n^{1+\log n+\cdots+(\log n)^{i-1}}\left|\gamma_{i+1}(G)\right|^{(\log n)^{i}}$, onde a base do logaritmo é 2 .

DemonstraÇÃo. ${ }^{1}$ Usaremos indução sobre $i$. O caso $i=1$ é o conteúdo do Lema 2.2 de [17], mas também pode ser obtido do Lema 2.4.6. Agora, para $i \geq 2$, consideremos o quociente $G / Z(G)$. Pelo Lema 2.4.6, temos que

$$
\left|\gamma_{i}(G / Z(G))\right| \leq n^{1+\log \left|\gamma_{i+1}(G)\right|}=n\left|\gamma_{i+1}(G)\right|^{\log n}
$$

Além disso, $A Z(G) / Z(G)$ é um subgrupo abeliano de índice finito em $G / Z(G)$. De fato, $m=[G / Z(G): A Z(G) / Z(G)]=[G: A Z(G)] \leq n$, que é finito por hipótese, e $A Z(G) / Z(G)$ é isomorfo ao grupo abeliano $A /(A \cap Z(G))$.

Da hipótese de indução aplicada ao grupo $G / Z(G)$ segue que

$$
\begin{aligned}
{\left[G / Z(G): Z_{i-1}(G / Z(G))\right] } & \leq m^{1+\log m+\cdots+(\log m)^{i-2}}\left|\gamma_{i}(G / Z(G))\right|^{(\log m)^{i-1}} \\
& \leq n^{1+\log n+\cdots+(\log n)^{i-2}}\left|\gamma_{i}(G / Z(G))\right|^{(\log n)^{i-1}}
\end{aligned}
$$

pois $1 \leq m \leq n$. Notando que $\left[G: Z_{i}(G)\right]=\left[G / Z(G): Z_{i-1}(G / Z(G))\right]$ e usando a fórmula (2.4.2), temos que

$$
\begin{aligned}
{\left[G: Z_{i}(G)\right] } & \leq n^{1+\log n+\cdots+(\log n)^{i-2}}\left(n\left|\gamma_{i+1}(G)\right|^{\log n}\right)^{(\log n)^{i-1}} \\
& =n^{1+\log n+\cdots+(\log n)^{i-2}} \cdot n^{(\log n)^{i-1}} \cdot\left|\gamma_{i+1}(G)\right|^{(\log n)^{i}}
\end{aligned}
$$

Isto conclui a indução e o teorema está provado.

\footnotetext{
${ }^{1}$ As demonstrações do Teorema 2.4.7 e do Lema 2.4.6 foram obtidas pelo autor em conjunto com sua orientadora.
} 


\subsection{Teorema de Hall}

Dizemos que um grupo $G$ é grupo finito-por-nilpotente se $G$ possui um subgrupo normal $N$ tal que $N$ é finito e $G / N$ é nilpotente. A condição que $G / N$ é nilpotente é equivalente a se ter $\gamma_{i+1}(G) \leq N$, para algum inteiro $i$. Portanto, um grupo é finito-por-nilpotente se, e somente se, $\gamma_{i+1}(G)$ é finito, para algum inteiro $i$.

Nesse sentido, o Teorema de Baer pode ser interpretado como: se algum termo da série central ascendente de um grupo $G$ tem índice finito então $G$ é finito-por-nilpotente. O Teorema de Hall mostra justamente o inverso: se $G$ é finito-por-nilpotente então algum termo da série central ascendente de $G$ tem índice finito. Mais precisamente, o Teorema de Hall diz que se $\gamma_{i+1}(G)$ é finito então $\left[G: Z_{2 i}(G)\right]$ é finito, sendo assim uma recíproca parcial do Teorema de Baer, já que os termos das série central ascendente envolvidos no dois enunciados não são os mesmos. Além disso, o Teorema de Hall mostra que um grupo finito-por-nilpotente é necessariamente nilpotente-por-finito.

A demonstração do Teorema de Hall depende de dois lemas. O primeiro é um caso particular de [11, Lemma 1].

Lema 2.5.1. Sejam $M$ e $N$ subgrupos normais de um grupo $G e$ um inteiro $n \geq 0$. Então $\left[M, N,{ }_{n} G\right] \leq \prod_{j=0}^{n}\left[\left[M,{ }_{j} G\right],\left[N,_{n-j} G\right]\right]$.

DemonstraçÃo. Argumentamos por indução sobre $n$, sendo óbvio o caso $n=0$. Assim, supondo que o resultado vale para $n \geq 0$, temos

$$
\left[M, N, n+1_{n} G\right]=\left[\left[M, N,_{n} G\right], G\right] \leq\left[\prod_{j=0}^{n}\left[\left[M,_{j} G\right],\left[N, n-j_{j} G\right]\right], G\right]
$$

Desse fato e do Corolário 1.2.7, segue que

$$
\left[M, N,_{n+1} G\right] \leq \prod_{j=0}^{n}\left[\left[M,,_{j} G\right],\left[N,_{n-j} G\right], G\right]
$$


onde a normalidade dos fatores é garantida pela Proposição 1.2.5. Aplicando o Lema 1.3.5 a cada fator do produto em (2.5.1)), temos que

$$
\begin{aligned}
{\left[M, N,_{n+1} G\right] } & \leq \prod_{j=0}^{n}\left[\left[M,_{j+1} G\right],\left[N,_{n-j} G\right]\right]\left[\left[M,{ }_{j} G\right],\left[N,_{n+1-j} G\right]\right] \\
& \leq \prod_{j=0}^{n+1}\left[\left[M,_{j} G\right],[N,(n+1)-j G]\right]
\end{aligned}
$$

e isso conclui a indução e a demonstração.

O segundo lema também é um caso particular de um resultado mais geral devido a Hall [11, Lemma 2].

Lema 2.5.2. Sejam $G$ um grupo e $C=C_{G}\left(\gamma_{i+1}(G)\right)$, onde $i \geq 0$. Se $m$ e $n$ são inteiros não negativos tais que $m+n \geq 2 i-1$, então $\left[C,{ }_{m} G, C\right] \leq Z_{n}(G)$.

DemonstraÇÃo. É suficiente mostrar que $\left[C,_{m} G, C,_{n} G\right]=1$. De fato, se isso vale, a Proposição 1.3 .3 garante que $\left[C,_{m} G, C\right] \leq Z_{n}(G)$. Pela Proposição 1.2.2, $C$ é normal em $G$. Logo $\left[C,_{m} G\right]$ também é normal em $G$, pela Proposição 1.2.5. Assim, podemos aplicar o Lema 2.5.1 com $M=\left[C,_{m} G\right]$ e $N=C$, e temos

$$
\left[\left[C,_{m} G\right], C,_{n} G\right] \leq \prod_{j=0}^{n}\left[\left[C,_{m+j} G\right],\left[C,_{n-j} G\right]\right] .
$$

Como $(m+j)+(n-j) \geq 2 i-1$, temos que $m+j \geq i$ ou $n-j \geq i$, para todo $0 \leq j \leq n$. Logo, $\left[C,_{m+j} G\right]$ ou $\left[C_{,_{n-j}} G\right]$ está contido em $\gamma_{i+1}(G)$. Portanto, $\left[\left[C,_{m+j} G\right],\left[C,_{n-j} G\right]\right] \leq\left[\gamma_{i+1}(G), C\right]=1$, para todo $0 \leq j \leq$ $n$. Segue de $(2.5 .2)$ que $\left[\left[C,_{m} G\right], C,_{n} G\right]=1$, como desejado.

Destacamos um corolário do resultado anterior. 
Corolário 2.5.3. Seja G um grupo cujo centro é trivial. Então os centralizadores em $G$ dos termos da série central descendente são abelianos.

DemonstraçÃo. Seja $i \geq 0$ e $C=C_{G}\left(\gamma_{i+1}(G)\right)$. Como $Z(G)=1$, temos que $Z_{k}(G)=1$, para todo $k \geq 0$. Tomando $m=0$ e $n=2 i-1$ no Lema 2.5.2, temos que $[C, C] \leq Z_{2 i-1}(G)=1$. Portanto, $C$ é abeliano.

Finalmente estamos prontos para dar a demonstração do Teorema de Hall.

Teorema 2.5.4. [11, Theorem 2] Seja G um grupo. Se $\gamma_{i+1}(G)$ é finito, para algum $i \geq 0$, então $\left[G: Z_{2 i}(G)\right]$ é finito. Além disso, $\left[G: Z_{2 i}(G)\right] \leq h\left(\left|\gamma_{i+1}(G)\right|\right)$, onde $h(n)=n^{\log _{2} n+\left(\log _{2} n\right)^{2 i}}$.

Demonstração. Se $i=0$ o resultado é trivial. Assim, considere $i \geq 1$. Primeiramente, segundo a Proposição 1.1.4, existem um inteiro $t \leq \log _{2}\left|\gamma_{i+1}(G)\right|$ e elementos $x_{1}, \ldots, x_{t} \in \gamma_{i+1}(G)$ tais que $\gamma_{i+1}(G)=\left\langle x_{1}, \ldots, x_{t}\right\rangle$. Seja $C=C_{G}\left(\gamma_{i+1}(G)\right)=C_{G}\left(x_{1}\right) \cap \cdots \cap C_{G}\left(x_{t}\right)$ e observe que, para todo $x \in \gamma_{i+1}(G)$, tem-se $x^{G} \subseteq \gamma_{i+1}(G)$ e, então, $\left[G: C_{G}(x)\right]=\left|x^{G}\right| \leq\left|\gamma_{i+1}(G)\right|$. Disso, e da Proposição 1.0.2, segue que $[G: C] \leq \prod_{j=1}^{t}\left[G: C_{G}\left(x_{j}\right)\right] \leq\left|\gamma_{i+1}(G)\right|^{t}$. Portanto,

$$
[G: C] \leq\left|\gamma_{i+1}(G)\right|^{\log _{2}\left|\gamma_{i+1}(G)\right|}<\infty
$$

Vamos supor sem perda de generalidade que $[G: C]>1$, porque caso contrário $\gamma_{i+1}(G) \leq Z(G)$ e, assim, $\gamma_{i+2}(G)=1$. Isto implica que $G=Z_{i+1}(G)$ e disso segue que $\left[G: Z_{2 i}(G)\right]=1$.

Como $C$ é um subgrupo próprio de índice finito em $G$, a Proposição 1.1.3 garante que existem um inteiro $r \leq \log _{2}[G: C]$ e elementos 
$g_{1}, \ldots, g_{r} \in G$ tais que $G=\left\langle g_{1}, \ldots, g_{r}, C\right\rangle$. Segue de (2.5.3) que

$$
r \leq \log _{2}\left(\left|\gamma_{i+1}(G)\right|^{\log _{2}\left|\gamma_{i+1}(G)\right|}\right)=\left(\log _{2}\left|\gamma_{i+1}(G)\right|\right)^{2}
$$

Como $\gamma_{i+1}(G)$ é normal em $G$, temos que $C$ é normal em $G$, pela Proposição 1.2.2. Assim, qualquer que seja $k \geq 0$, temos que $\left[C,{ }_{k} G\right]$ é normal em $G$, pela Proposição 1.2.5. Para cada $0 \leq s \leq i$, definimos

$$
Q_{s}=\frac{\left[C,{ }_{s} G\right]}{\left[C,{ }_{s} G\right] \cap Z_{2 i-s}(G)} .
$$

Mostraremos, por indução reversa sobre $s$, que cada $Q_{s}$ é finito e que $\left|Q_{s}\right| \leq\left|\gamma_{i+1}(G)\right|^{r^{i-s}}$.

Note que, $Q_{i}$ é isomorfo a $\left[C{ }_{i} G\right] Z_{i}(G) / Z_{i}(G)$, que é subgrupo de $\gamma_{i+1}(G) Z_{i}(G) / Z_{i}(G)$, pois $\left[C{ }_{i} G\right] \leq \gamma_{i+1}(G)$. Como $\gamma_{i+1}(G) Z_{i}(G) / Z_{i}(G)$ é isomorfo a um fator do grupo $\gamma_{i+1}(G)$, segue que $\left|Q_{i}\right| \leq\left|\gamma_{i+1}(G)\right|$, que é finito por hipótese. Estabelecemos, assim, a base de indução. Agora, supondo que por indução $\left|Q_{s+1}\right| \leq\left|\gamma_{i+1}(G)\right|^{r^{i-s-1}}$, mostraremos que $\left|Q_{s}\right| \leq\left|\gamma_{i+1}(G)\right|^{r^{i-s}}$.

Para todo $g \in G$, a aplicação $\varphi_{g}$, definida abaixo, é um homomorfismo do grupo $\left[C,,_{s} G\right]$ no grupo finito $Q_{s+1}$ :

$$
\begin{aligned}
\varphi_{g}:\left[C,{ }_{s} G\right] & \longrightarrow \frac{\left[C,{ }_{s+1} G\right]}{\left[C,_{s+1} G\right] \cap Z_{2 i-s-1}(G)} . \\
x & \longmapsto[x, g]\left(\left[C,_{s+1} G\right] \cap Z_{2 i-s-1}(G)\right)
\end{aligned}
$$

De fato, para quaisquer $x, y \in\left[C,{ }_{s} G\right]$, temos $[x y, g]=[x, g][x, g, y][y, g]$, sendo que $[x, g, y] \in\left[C,_{s+1} G\right] \cap Z_{2 i-s-1}(G)$, pois por um lado o comutador $[x, g, y] \in\left[C,_{s+1} G, C\right] \leq Z_{2 i-s-1}(G)$, pelo Lema 2.5.2, e por outro lado $[x, g, y] \in\left[C,_{s+1} G\right]$. Assim, módulo $\left[C_{s_{s+1}} G\right] \cap Z_{2 i-s-1}(G)$, temos que $[x y, g]=[x, g][y, g]$, ou seja, $\varphi_{g}(x y)=\varphi_{g}(x) \varphi_{g}(y)$. 
Pelo Teorema do Isomorfismo, para cada $g \in G$, temos

$$
\left[\left[C,{ }_{s} G\right]: \operatorname{Ker}\left(\varphi_{g}\right)\right] \leq\left|Q_{s+1}\right|<\infty
$$

Lembre-se que $G=\left\langle g_{1}, \ldots, g_{r}, C\right\rangle$ e defina $H_{s}=\bigcap_{k=1}^{r} \operatorname{Ker}\left(\varphi_{g_{k}}\right)$. Da Proposição 1.0.2 e da fórmula (2.5.5) segue que $\left[\left[C,,_{s} G\right]: H_{s}\right] \leq\left|Q_{s+1}\right|^{r}$. Pela hipótese de indução, $\left|Q_{s+1}\right| \leq\left|\gamma_{i+1}(G)\right|^{r^{i-s-1}}$ e, assim,

$$
\left[\left[C,_{s} G\right]: H_{s}\right] \leq\left|\gamma_{i+1}(G)\right|^{i-s}
$$

Agora mostramos que $H_{s} \leq\left[C,{ }_{s} G\right] \cap Z_{2 i-s}(G)$. Note que, para cada $1 \leq k \leq r$, temos que $\left[H_{s}, g_{k}\right] \leq Z_{2 i-s-1}(G)$, pela definição de $H_{s}$. Além disso, $\left[H_{s}, C\right] \leq\left[C,{ }_{s} G, C\right] \leq Z_{2 i-s-1}(G)$, pelo Lema 2.5.2. Como $G=\left\langle g_{1}, \ldots, g_{r}, C\right\rangle$, segue que $\left[H_{s}, G\right] \leq Z_{2 i-s-1}(G)$, ou seja, $H_{s} \leq Z_{2 i-s}(G)$. Assim, $H_{s} \leq\left[C,{ }_{s} G\right] \cap Z_{2 i-s}(G)$ e, portanto, temos que $\left|Q_{s}\right|=\left[\left[C,_{s} G\right]:\left[C,_{s} G\right] \cap Z_{2 i-s}(G)\right] \leq\left[\left[C,_{s} G\right]: H_{s}\right]$. Da relação (2.5.6) segue que $\left|Q_{s}\right| \leq\left|\gamma_{i+1}(G)\right|^{r^{i-s}}$, o que conclui a indução.

Para terminar, notamos que $\left[C: C \cap Z_{2 i}(G)\right]=\left|Q_{0}\right| \leq\left|\gamma_{i+1}(G)\right|^{r^{i}}$. Então, pela desigualdade (2.5.4), temos que

$$
\left[C: C \cap Z_{2 i}(G)\right] \leq\left|\gamma_{i+1}(G)\right|^{\left(\log _{2}\left|\gamma_{i+1}(G)\right|\right)^{2 i}} .
$$

Usando as desigualdades (2.5.3) e (2.5.7), concluímos a demonstração observando que:

$$
\begin{aligned}
{\left[G: Z_{2 i}(G)\right] } & \leq[G: C] \cdot\left[C: C \cap Z_{2 i}(G)\right] \\
& \leq\left|\gamma_{i+1}(G)\right|^{\log _{2}\left|\gamma_{i+1}(G)\right|} \cdot\left|\gamma_{i+1}(G)\right|^{\left(\log _{2}\left|\gamma_{i+1}(G)\right|\right)^{2 i}} \\
& =h\left(\left|\gamma_{i+1}(G)\right|\right) .
\end{aligned}
$$


A proposição abaixo, que é consequência do Teorema de Baer e que foi demonstrada por Hall em [11], fornece uma condição suficiente para que $\gamma_{i+1}(G)$ seja finito.

Proposição 2.5.5. [11, Corollary] Seja $G$ um grupo. Se G possui um subgrupo normal $M$ de indice finito tal que, para algum $i \geq 0$, $\left[M,{ }_{i} G\right]$ é finito então $\gamma_{i+1}(G)$ é finito.

DemonstraçÃo. Trabalharemos com o quociente $G /\left[M_{, i} G\right]$. Note que $\left[M /\left[M,{ }_{i} G\right],{ }_{i}\left(G /\left[M,{ }_{i} G\right]\right)\right]=\left[M,_{i} G\right] /\left[M,_{i} G\right]=1 \mathrm{e}$, portanto, temos que $M /\left[M,_{i} G\right] \leq Z_{i}\left(G /\left[M,,_{i} G\right]\right)$. Além disso, como $[G: M]$ é finito, temos que $\left[G /\left[M,_{i} G\right]: M /\left[M,_{i} G\right]\right]$ é finito. Logo, concluímos que $\left[G /\left[M,{ }_{i} G\right]: Z_{i}\left(G /\left[M,_{i} G\right]\right)\right]$ é finito. Segue do Teorema 2.3.2 de Baer que $\gamma_{i+1}\left(G /\left[M,_{i} G\right]\right)=\gamma_{i+1}(G) /\left[M_{, i} G\right]$ é finito. Isto, junto com a hipótese de que $\left[M,_{i} G\right]$ é finito, nos garante que $\gamma_{i+1}(G)$ é finito.

A seguir, vamos apresentar outros resultados sobre a recíproca do Teorema de Baer obtidos como consequências do Teorema 2.5.4 de Hall.

Dado um grupo $G$, um subgrupo próprio $M$ de $G$ é dito maximal se, sempre que $M \leq H \leq G$ tem-se que $H=G$ ou $H=M$. O subgrupo de Frattini $\Phi(G)$ é a interseção dos subgrupos maximais de $G$, com a convenção de que $\Phi(G)=G$ caso $G$ não tenha nenhum subgrupo maximal. O resultado seguinte dá uma relação entre o subgrupo derivado, o centro e o subgrupo de Frattini de um grupo qualquer. Ele é um caso particular de um resultado mais geral devido a Gaschütz (veja [16, Proposition 2.6]).

Lema 2.5.6. Seja $G$ um grupo. Então $G^{\prime} \cap Z(G) \leq \Phi(G)$.

DemonstraÇÃo. Basta mostrar que $G^{\prime} \cap Z(G)$ está contido em todo subgrupo maximal de $G$. Sendo assim, seja $M$ um subgrupo 
maximal arbitrário do grupo $G$. Se $Z(G) \subseteq M$ então $G^{\prime} \cap Z(G) \subseteq M$. Se $Z(G) \nsubseteq M$ então $G=M Z(G)$, pois $M$ é maximal. Segue das fórmulas (1.2.1) e (1.2.2) que $G^{\prime}=[M Z(G), M Z(G)]=[M, M] \leq M$ e, assim, $G^{\prime} \cap Z(G) \leq M$.

A proposição abaixo diz que todo grupo finito-por-nilpotente, cujo subgrupo de Frattini é trivial, é central-por-finito.

Proposição 2.5.7. [9, Lemma 2.1] Seja $G$ um grupo tal que $\Phi(G)=$ 1. Se, para algum $i \geq 1, \gamma_{i+1}(G)$ é finito então $[G: Z(G)]$ é finito. Em particular, se $G^{\prime}$ é finito então $[G: Z(G)]$ é finito.

Demonstração. Pela Proposição 2.5.6, $G^{\prime} \cap Z(G) \leq \Phi(G)$. Então $G^{\prime} \cap Z(G)=1$ e, assim, $\left[Z_{2}(G), G\right] \leq G^{\prime} \cap Z(G)=1$. Portanto, $Z_{2}(G) \leq Z(G)$ e, assim, $Z_{2}(G)=Z(G)$. É fácil ver, por indução sobre $j$, que $Z_{j}(G)=Z(G)$, qualquer que seja $j \geq 1$. Pelo Teorema de Hall, temos que $\left[G: Z_{2 i}(G)\right]$ é finito, e o resultado segue.

Halasi e Podoski ainda mostram [9, Theorem 1.1] que, se $\Phi(G)=1$ e $G^{\prime}$ é finito então $[G: Z(G)] \leq\left|G^{\prime}\right|^{2}$ e a igualdade vale se, e somente se, $G$ for abeliano.

No próximo resultado, que é obtido combinando o Teorema de Hall e o Teorema 2.4.4, Hatamian, Hassanzadeh e Kayvanfar provam que a recíproca do Teorema de Baer vale para um grupo $G$ tal que $Z_{2 i}(G) / Z_{i}(G)$ é finitamente gerado, para algum $i \geq 0$.

Proposição 2.5.8. [14, Theorem 1] Seja G um grupo tal que, para algum $i \geq 0$, o quociente $Z_{2 i}(G) / Z_{i}(G)$ é finitamente gerado. Se $\gamma_{i+1}(G)$ é finito então $\left[G: Z_{i}(G)\right]$ é finito.

Demonstração. Pelo Teorema de Hall, $G / Z_{2 i}(G)$ é finito e, em particular, finitamente gerado. Assim, pela Proposição 1.1.1, $G / Z_{i}(G)$ 
é finitamente gerado. Portanto, segue do Teorema 2.4.4 que $\left[G: Z_{i}(G)\right]$ é finito.

Outra classe de grupos que satisfazem a recíproca do Teorema de Baer é apresentada no teorema abaixo, cuja demonstração foi dada por Hall em [11].

TeOrema 2.5.9. Seja $G$ um grupo tal que $\gamma_{i+1}(G) \cap Z(G)=1$, para algum $i \geq 0$. Se $\gamma_{i+1}(G)$ é finito então $\left[G: Z_{i}(G)\right]$ é finito.

DemonstraçÃo. Pela Proposição 1.3.4, $\gamma_{i+1}(G) \cap Z_{n}(G)=1$, para todo $n \geq 1$. Assim, $\left[Z_{n}(G){ }_{i} G\right] \leq \gamma_{i+1}(G) \cap Z_{n}(G)=1$ e, portanto, $Z_{n}(G) \leq Z_{i}(G)$, para todo $n \geq 1$. Em particular, $Z_{2 i}(G)=Z_{i}(G)$. Do Teorema de Hall concluímos que $\left[G: Z_{2 i}(G)\right]=\left[G: Z_{i}(G)\right]$ é finito.

Em [20] Macdonald provou a recíproca do Teorema de Baer para grupos que satisfazem a condição minimal. Lembramos que um grupo $G$ satisfaz a condição minimal se não possui nenhuma cadeia descendente infinita de subgrupos $G>H_{1}>H_{2}>\cdots$. Para provar esse resultado vamos precisar do seguinte teorema devido a Baer sobre grupos nilpotentes que satisfazem a condição minimal.

TeOrema 2.5.10. [26, Theorem 3.14] Se um grupo nilpotente $G$ satisfaz a condição minimal então $[G: Z(G)]$ é finito.

A demonstração desse teorema foge do escopo desta dissertação e por isso não a apresentaremos aqui.

TeOrema 2.5.11. [20, p. 178] Seja G um grupo que satisfaz a condição minimal. Se, para algum $i \geq 0, \gamma_{i+1}(G)$ é finito então o indice $\left[G: Z_{i}(G)\right]$ é finito. 
Demonstração. Pelo Teorema de Hall, $\left[G: Z_{2 i}(G)\right]$ é finito. Como $Z_{2 i}(G)$ é nilpotente e também satisfaz a condição minimal, pelo Teorema 2.5.10, o grupo abeliano $Z\left(Z_{2 i}(G)\right)$ tem índice finito em $Z_{2 i}(G)$. Assim, temos que $\left[G: Z\left(Z_{2 i}(G)\right)\right]=\left[G: Z_{2 i}(G)\right]\left[Z_{2 i}(G): Z\left(Z_{2 i}(G)\right)\right]$ é finito. Segue do Teorema 2.4.7 que $\left[G: Z_{i}(G)\right]$ é finito.

Concluímos destacando um último resultado de Macdonald na mesma linha dos resultados desta seção.

Teorema 2.5.12. [20, p. 178] Seja G um grupo metabeliano. Se, para algum $i \geq 0, \gamma_{i+1}(G)$ é finito então $\left[G: Z_{i+1}(G)\right]$ é finito.

Note que, sendo $G$ metabeliano e $\gamma_{i+1}(G)$ finito, o índice $\left[G: Z_{i}(G)\right]$ não é necessariamente finito, como mostra o Exemplo 2.2.1, para o caso $i=1$. 


\section{CAPíTULO 3}

\section{Generalizando o Teorema de Hall}

Neste capítulo trataremos dos resultados obtidos por FernándezAlcober e Morigi no artigo [7]. O objetivo do artigo é provar uma generalização do Teorema 2.5.4 de Hall, que afirma que $\left[G: Z_{2 i}(G)\right]$ é finito caso $\gamma_{i+1}(G)$ seja finito, para algum $i \geq 1$.

Uma forma mais forte do Teorema de Hall é conhecida para $i=1$ : é suficiente assumir que $\left[G^{\prime}: G^{\prime} \cap Z(G)\right]$ é finito para concluir que $\left[G: Z_{2}(G)\right]$ é finito. Isaacs $[\mathbf{1 7}]$ foi quem primeiro obteve uma limitação de $\left[G: Z_{2}(G)\right]$ em função de $\left[G^{\prime}: G^{\prime} \cap Z(G)\right]$, quando $G$ é finito. Posteriormente, Fernández-Alcober e Moretó [6, Theorem E] mostraram que o resultado é válido quando $G$ é um grupo nilpotente qualquer (possivelmente infinito). Independentemente, Podoski e Szegedy $[\mathbf{2 4}$, Theorem 1] provaram o resultado para um grupo $G$ qualquer. O objetivo de [7] é generalizar este resultado para valores arbitrários de $i$ na forma do teorema abaixo.

Teorema A. [7, Theorem A] Sejam $G$ um grupo e $i \geq 0$ tais que $\left[\gamma_{i+1}(G): \gamma_{i+1}(G) \cap Z_{i}(G)\right]$ é finito. Então $\left[G: Z_{2 i}(G)\right]$ é finito e limitado em função de $\left[\gamma_{i+1}(G): \gamma_{i+1}(G) \cap Z_{i}(G)\right]$.

Forneceremos exemplos para ver que o Teorema A, em certo sentido, é a melhor generalização possível do Teorema de Hall.

Observamos que, usando o Teorema de Hall, já podíamos mostrar que se $\left[\gamma_{i+1}(G): \gamma_{i+1}(G) \cap Z_{i}(G)\right]$ é finito então $\left[G: Z_{3 i}(G)\right]$ é finito. De fato, $\left[\gamma_{i+1}(G): \gamma_{i+1}(G) \cap Z_{i}(G)\right]$ é igual à ordem de $\gamma_{i+1}\left(G / Z_{i}(G)\right)$. 
Então, segue do Teorema de Hall aplicado ao quociente $G / Z_{i}(G)$, que $\left[G / Z_{i}(G): Z_{2 i}\left(G / Z_{i}(G)\right)\right]$ é finito. Portanto, $\left[G: Z_{3 i}(G)\right]$ é finito, pois pela Proposição 1.3 .8 é igual a $\left[G / Z_{i}(G): Z_{2 i}\left(G / Z_{i}(G)\right)\right]$.

Observamos ainda, que Macdonald em [20, Corollary 2] já havia mostrado que se, para algum $i \geq 0,\left[\gamma_{i+1}(G): \gamma_{i+1}(G) \cap Z(G)\right]$ é finito então $\left[G: Z_{2 i}(G)\right]$ é finito.

\subsection{Seguindo os passos de P. Hall}

No Teorema de Schur vimos que existe uma função $f(n)$ tal que se $G$ é um grupo com $[G: Z(G)]$ finito então $\left|G^{\prime}\right| \leq f([G: Z(G)])$. Em uma situação como essa dizemos que $\left|G^{\prime}\right|$ é limitada em função de $[G: Z(G)]$. Nesta seção usaremos muitas vezes essa expressão.

Vamos começar com um resultado técnico que será essencial para provar o Teorema A.

Proposição 3.1.1. [7, Proposition 2.3] Sejam um grupo G e um inteiro $s \geq 1$ tais que $\left[\gamma_{s}(G): \gamma_{s}(G) \cap Z(G)\right]$ é finito. Então o índice $\left[G: C_{G}\left(\gamma_{s}(G)\right)\right]$ é limitado em função de $\left[\gamma_{s}(G): \gamma_{s}(G) \cap Z(G)\right]$.

DemonstraÇÃo. Denotamos, por simplicidade, $Z=\gamma_{s}(G) \cap Z(G)$ e $n=\left[\gamma_{s}(G): Z\right]$. A chave desta demonstração é o Corolário 1.6.7. Para que ele possa ser usado, encontraremos um subgrupo finitamente gerado $U$ de $G$ tal que $\gamma_{s}(G)=\gamma_{s}(U) Z$ e mostraremos que, de fato, todos os termos da série central descendente de $U$ são finitamente gerados.

Sejam $X=\left\{\left[g_{1}, \ldots, g_{s}\right] \mid g_{1}, \ldots, g_{s} \in G\right\}$, o conjunto dos comutadores de peso $s$, e $\bar{X}=\{x Z \mid x \in X\}$, sua imagem em $G / Z$. Como, por hipótese, $\left[\gamma_{s}(G): Z\right]=n$, temos que $m=|\bar{X}| \leq n$. Dessa forma, existem $x_{1}=\left[g_{11}, \ldots, g_{1 s}\right], \ldots, x_{m}=\left[g_{m 1}, \ldots, g_{m s}\right]$ tais 
que $\bar{X}=\left\{x_{1} Z, \ldots, x_{m} Z\right\}$. Note que, $\gamma_{s}(G) / Z=\langle\bar{X}\rangle$, então temos $\gamma_{s}(G)=\left\langle x_{1}, \ldots, x_{m}, Z\right\rangle$. Seja $U=\left\langle g_{i j} \mid 1 \leq i \leq m, 1 \leq j \leq s\right\rangle$. Como $x_{1}, \ldots, x_{m} \in \gamma_{s}(U)$, temos que $\gamma_{s}(G)=\left\langle x_{1}, \ldots, x_{m}, Z\right\rangle \leq \gamma_{s}(U) Z$. Por outro lado, $\gamma_{s}(U) \leq \gamma_{s}(G)$ e $Z \leq \gamma_{s}(G)$, logo $\gamma_{s}(U) Z \leq \gamma_{s}(G)$. Portanto, $\gamma_{s}(G)=\gamma_{s}(U) Z$, onde o número de geradores de $U$ é limitado em função de $n$.

Agora mostraremos que $\gamma_{2 s}(G)$ é finito, aplicando os Teoremas de Hall e de Baer. Note que a ordem de $\gamma_{s}(G / Z(G))$ é igual ao índice $\left[\gamma_{s}(G): \gamma_{s}(G) \cap Z(G)\right]=n$. Assim, pelo Teorema 2.5.4 de Hall, $\left[G / Z(G): Z_{2(s-1)}(G / Z(G))\right] \leq h(n)$, onde $h(n)=n^{\log _{2} n+\left(\log _{2} n\right)^{2(s-1)}}$. Pela Proposição 1.3.8, temos que $Z_{2 s-2}(G / Z(G))=Z_{2 s-1}(G) / Z(G)$. Então $\left[G: Z_{2 s-1}(G)\right]=\left[G / Z(G): Z_{2 s-1}(G) / Z(G)\right] \leq h(n)$. Portanto, pelo Teorema 2.3.2 de Baer, $\gamma_{2 s}(G)$ é finito e tem ordem limitada em função de $n$.

Como o número de geradores de $U$ e a ordem de $\gamma_{2 s}(U)$ são limitados em função de $n$, segue do Corolário 1.3.11 que cada termo da série central descendente de $U$ é gerado por um número finito de elementos, sendo esse número limitado em função de $n$.

Mostraremos agora que, para todo $1 \leq j \leq s$, existe um subgrupo $H_{j} \leq \gamma_{j}(G)$ tal que $\left[\gamma_{s-j+1}(U), H_{j}\right]=1$ e que $\left[\gamma_{j}(G): H_{j}\right]$ é finito e limitado em função de $n$. Em particular, se isso é verdade, existe um subgrupo $H_{1} \leq G$ tal que $\left[\gamma_{s}(G), H_{1}\right]=\left[\gamma_{s}(U) Z, H_{1}\right]=\left[\gamma_{s}(U), H_{1}\right]=1$ e que $\left[G: H_{1}\right]$ é finito e limitado em função de $n$. Isto implica que $H_{1} \leq C_{G}\left(\gamma_{s}(G)\right) \leq G$ e, assim, $\left[G: C_{G}\left(\gamma_{s}(G)\right)\right]$ é finito e limitado em função de $n$. Isto completa a demonstração do teorema. 
Argumentamos por indução reversa sobre $j$. Quando $j=s$, considere $H_{s}=Z=\gamma_{s}(G) \cap Z(G)$, que tem índice finito $n$ em $\gamma_{s}(G)$, por hipótese, e note que $\left[\gamma_{1}(U), H_{s}\right] \leq[U, Z(G)]=1$, como desejado.

Supondo, por indução, que existe um subgrupo $H_{j+1} \leq \gamma_{j+1}(G)$ tal que $\left[\gamma_{s-j}(U), H_{j+1}\right]=1$ e que $\left[\gamma_{j+1}(G): H_{j+1}\right]$ é finito e limitado em função de $n$, mostraremos que existe $H_{j} \leq \gamma_{j}(G)$ tal que $\left[\gamma_{s-j+1}(U), H_{j}\right]=\left[\gamma_{s-j}(U), U, H_{j}\right]=1$ e que $\left[\gamma_{j}(G): H_{j}\right]$ é finito e limitado em termos de $n$.

Pelo Lema 1.3.5 dos Três Subgrupos, é suficiente que $H_{j}$ satisfaça $\left[H_{j}, \gamma_{s-j}(U), U\right]=1=\left[H_{j}, U, \gamma_{s-j}(U)\right]$. Para isso, definiremos $H_{j}$ como a interseção de dois subgrupos específicos $K_{j}$ e $L_{j}$, ambos de índice finito em $\gamma_{s}(G)$, tais que $\left[K_{j}, \gamma_{s-j}(U), U\right]=1=\left[L_{j}, U, \gamma_{s-j}(U)\right]$.

Primeiro vemos como definir $K_{j}$. Defina $K_{j}=C_{\gamma_{j}(G)}\left(\gamma_{s-j}(U) Z / Z\right)$. Como $\left[\gamma_{j}(G), \gamma_{s-j}(U)\right] Z / Z \leq \gamma_{s}(G) Z / Z=\gamma_{s}(G) / Z$, que tem ordem $n$ e o número de geradores de $\gamma_{s-j}(U)$ é limitado em função de $n$, pelo Corolário 1.6.7, temos que o índice de $K_{j}$ em $\gamma_{j}(G)$ é finito e limitado em função de $n$. Note também que

$$
\left[K_{j}, \gamma_{s-j}(U), U\right] \leq[Z, U]=1
$$

Vamos definir agora um subgrupo auxiliar que será usado na construção do subgrupo $L_{j}$. Defina $D_{j+1}=C_{\gamma_{j+1}(G)}\left(\gamma_{s-j}(U)\right)$. Pela hipótese de indução, existe $H_{j+1} \leq \gamma_{j+1}(G)$ tal que $\left[H_{j+1}, \gamma_{s-j}(U)\right]=1$ e que $\left[\gamma_{j+1}(G): H_{j+1}\right]$ é finito e limitado em função de $n$. Assim, temos que $H_{j+1} \leq D_{j+1} \leq \gamma_{j+1}(G)$ e, portanto, o índice de $D_{j+1}$ em $\gamma_{j+1}(G)$ é finito e limitado em função de $n$.

No que segue, trabalharemos com quocientes por $D_{j+1}$, mas antes temos que mostrar que $D_{j+1}$ é normal em $U \gamma_{j}(G)$. Por um lado, 
pelo Lema 1.3.5, $\left[D_{j+1}, U, \gamma_{s-j}(U)\right]=1$, pois $\left[D_{j+1}, \gamma_{s-j}(U), U\right]=1 \mathrm{e}$ $\left[\gamma_{s-j}(U), U, D_{j+1}\right]=1$. Assim, $\left[D_{j+1}, U\right] \leq D_{j+1}$ e a Proposição 1.2.1 garante que $U \leq N_{G}\left(D_{j+1}\right)$. Por outro lado, $\left[\gamma_{j}(G), \gamma_{s-j}(U), D_{j+1}\right] \leq$ $\left[\gamma_{s}(G), D_{j+1}\right]=\left[\gamma_{s}(U) Z, D_{j+1}\right] \leq\left[\gamma_{s-j}(U) Z, D_{j+1}\right]=1$ e, além disso, $\left[\gamma_{s-j}(U), D_{j+1}, \gamma_{j}(G)\right]=1$, pela definição de $D_{j+1}$. Do Lema 1.3 .5 segue que $\left[D_{j+1}, \gamma_{j}(G), \gamma_{s-j}(U)\right]=1$. Assim, $\left[D_{j+1}, \gamma_{j}(G)\right] \leq D_{j+1}$ e, pela Proposição 1.2.1, temos que $\gamma_{j}(G) \leq N_{G}\left(D_{j+1}\right)$. Concluímos que $U \gamma_{j}(G) \leq N_{G}\left(D_{j+1}\right)$, ou seja, $D_{j+1}$ é normal em $U \gamma_{j}(G)$.

Considere o grupo quociente $U \gamma_{j}(G) / D_{j+1}$. Defina, finalmente, $L_{j}=C_{\gamma_{j}(G)}\left(U D_{j+1} / D_{j+1}\right)$. Observe que $\left[\gamma_{j}(G), U\right] D_{j+1} / D_{j+1}$ é subgrupo de $\gamma_{j+1}(G) / D_{j+1}$, que é finito e limitado em função de $n$, como vimos acima. Sendo o número de geradores de $U$ limitado em função de $n$, segue do Corolário 1.6.7 que o índice de $L_{j}$ em $\gamma_{j}(G)$ é finito e limitado em função de $n$. Note finalmente que, pelas definições de $L_{j}$ e de $D_{j+1}$,

$$
\left[L_{j}, U, \gamma_{s-j}(U)\right] \leq\left[D_{j+1}, \gamma_{s-j}(U)\right]=1
$$

Como já havíamos adiantado, definimos $H_{j}=K_{j} \cap L_{j}$ e notamos que o índice de $H_{j}$ em $\gamma_{j}(G)$ é finito e limitado em termos de $n$, pela Proposição 1.0.2, pois os índices de $K_{j}$ e de $L_{j}$ em $\gamma_{j}(G)$ são finitos e limitados em função de $n$. Agora, pelas equações (3.1.1) e (3.1.2), temos $\left[H_{j}, \gamma_{s-j}(U), U\right]=1=\left[H_{j}, U, \gamma_{s-j}(U)\right]$. Portanto, pelo Lema 1.3.5, $\left[\gamma_{s-j}(U), U, H_{j}\right]=\left[\gamma_{s-j+1}(U), H_{j}\right]=1$, o que mostra que $H_{j}$ possui as propriedades desejadas. Isto completa a indução e a demonstração.

Corolário 3.1.2. [7, Proposition 2.3] Sejam G um grupo e um inteiro $s \geq 1$ tais que $\left[\gamma_{s}(G): \gamma_{s}(G) \cap Z(G)\right]$ é finito. Então $\gamma_{s+1}(G)$ é finito e limitado em função de $\left[\gamma_{s}(G): \gamma_{s}(G) \cap Z(G)\right]$. 
Demonstração. Seja $n=\left[\gamma_{s}(G): \gamma_{s}(G) \cap Z(G)\right]$. Pela Proposição 3.1.1, temos que $\left[G: C_{G}\left(\gamma_{s}(G)\right)\right]$ é finito e limitado em função de $n$. Aplicando o Teorema 2.3.1 com $M=C_{G}\left(\gamma_{s}(G)\right), H=G$, $N=\gamma_{s}(G) \cap Z(G)$ e $K=\gamma_{s}(G)$ concluímos que $\left[\gamma_{s}(G), G\right]=\gamma_{s+1}(G)$ é finito com ordem limitada em função de $n$.

Ressaltamos que o Corolário 3.1.2 já aparece como o Teorema 1 do artigo [20] de Macdonald, com uma demonstração diferente da que apresentamos aqui.

Vejamos, agora, que a Proposição 3.1.1 e o Corolário 3.1.2 não são válidos para um subgrupo normal qualquer no lugar de $\gamma_{s}(G)$. Mais precisamente, se $N$ é um subgrupo normal de $G$ tal que $[N: N \cap Z(G)]$ é finito, não necessariamente temos que $\left[G: C_{G}(N)\right]$ é finito ou que $[N, G]$ é finito.

Sejam $p$ um primo e $N$ e $A$ dois $p$-grupos abelianos elementares tais que $N=\left\langle x_{0}, x_{1}, x_{2}, \ldots\right\rangle$ e $A=\left\langle a_{1}, a_{2}, \ldots\right\rangle$. Definimos a seguinte ação de $A$ em $N$ : para todo $i \geq 1$, a ação de $a_{i}$ é tal que $x_{0}^{a_{i}}=x_{0} x_{i}$ e $x_{j}^{a_{i}}=x_{j}$, quando $j \geq 1$. No produto semidireto $G=N \rtimes A$, para todo $i \geq 1$, temos $\left[x_{0}, a_{i}\right]=x_{i}$ e $\left[x_{j}, a_{i}\right]=1$, quando $j \geq 1$. Assim, é fácil ver que, $[N, G]=\left\langle x_{1}, x_{2}, \ldots\right\rangle$, que $C_{G}(N)=C_{G}\left(x_{0}\right)=N$ e que $Z(G)=\left\langle x_{1}, x_{2}, \ldots\right\rangle$. Concluímos que $[N: N \cap Z(G)]=p$, mas tanto $\left[G: C_{G}(N)\right]$ quanto $[N, G]$ são infinitos. Compare este exemplo com o Exemplo 2.2.1 e note o quanto são parecidos.

No que segue, precisaremos de uma identidade de grupos que deduziremos aqui. Sejam $G$ um grupo, $s \geq 1$ e $t \geq 0$. Note, por um lado, que $\gamma_{s}(G) /\left(\gamma_{s}(G) \cap Z_{t}(G)\right)$ é isomorfo a $\gamma_{s}\left(G / Z_{t}(G)\right)$. Por outro lado, $G / Z_{t}(G)$ é isomorfo a $\left(G / Z_{t-1}(G)\right) /\left(Z_{t}(G) / Z_{t-1}(G)\right)$ e, pela Proposição 1.3.8, $Z_{t}(G) / Z_{t-1}(G)=Z\left(G / Z_{t-1}(G)\right)$. Assim, $\gamma_{s}\left(G / Z_{t}(G)\right)$ é 
isomorfo a $\gamma_{s}\left(\left(G / Z_{t-1}(G)\right) / Z\left(G / Z_{t-1}(G)\right)\right)$. Portanto,

$$
\frac{\gamma_{s}(G)}{\gamma_{s}(G) \cap Z_{t}(G)} \cong \frac{\gamma_{s}\left(G / Z_{t-1}(G)\right)}{\gamma_{s}\left(G / Z_{t-1}(G)\right) \cap Z\left(G / Z_{t-1}(G)\right)}
$$

O próximo teorema [7, Theorem B] é um resultado técnico usado na demonstração do Teorema A, mas pode ser interessante por si só.

Teorema B. Seja $G$ um grupo tal que $\left[\gamma_{s}(G): \gamma_{s}(G) \cap Z_{t}(G)\right]$ é finito, para s e $t$ inteiros. Então, para todo inteiro $0 \leq j \leq t$, o indice $\left[\gamma_{s+j}(G): \gamma_{s+j}(G) \cap Z_{t-j}(G)\right]$ é finito e limitado em função de $\left[\gamma_{s}(G): \gamma_{s}(G) \cap Z_{t}(G)\right]$.

Demonstração. Defina $n=\left[\gamma_{s}(G): \gamma_{s}(G) \cap Z_{t}(G)\right]$. Argumentamos por indução sobre $t$. Para $t=0$ o resultado é trivial. Assumimos que o resultado é válido para $t-1$ e vamos prová-lo para $t$. Pela fórmula (3.1.3), temos que $\left[\gamma_{s}(\bar{G}): \gamma_{s}(\bar{G}) \cap Z(\bar{G})\right]=n$, onde $\bar{G}=G / Z_{t-1}(G)$. Segue do Corolário 3.1.2 que $\gamma_{s+1}(\bar{G})=\gamma_{s+1}\left(G / Z_{t-1}(G)\right)$ é finito e limitado em função de $n$. Isto é, $\left[\gamma_{s+1}(G): \gamma_{s+1}(G) \cap Z_{t-1}(G)\right]$ é finito e limitado em função de $n$. Então, pela hipótese de indução, para todo $0 \leq k \leq t-1$, temos que $\left[\gamma_{s+1+k}(G): \gamma_{s+1+k}(G) \cap Z_{t-1-k}(G)\right]$ é finito e limitado em função de $n$. Pondo $j=k+1$ o resultado segue.

Ressaltamos que o Teorema B já aparece em [20, Corollary 1] de Macdonald com uma demonstração similar.

Corolário 3.1.3. Seja $G$ um grupo. Se $\left[\gamma_{s}(G): \gamma_{s}(G) \cap Z_{t}(G)\right]$ é finito, para s e t inteiros, então $\gamma_{s+t}(G)$ é finito.

Basta considerar o caso $j=t$ no Teorema $\mathrm{B}$, que garante que $\left[\gamma_{s+t}(G): \gamma_{s+t}(G) \cap Z_{0}(G)\right]=\left|\gamma_{s+t}(G)\right|$ é finito. 
Notamos que, quando $s=i+1$ e $t=i$, o Corolário 3.1.3 garante que se $\left[\gamma_{i+1}(G): \gamma_{i+1}(G) \cap Z_{i}(G)\right]$ é finito então $\gamma_{2 i+1}(G)$ é finito.

Corolário 3.1.4. Sejam um grupo $G$ e inteiros $s$ e $t$ tais que $\left[\gamma_{s}(G): \gamma_{s}(G) \cap Z_{t}(G)\right]$ é finito. Então $\left[G: C_{G}\left(\gamma_{s}(G) /\left(\gamma_{s}(G) \cap Z_{t-1}(G)\right)\right]\right.$ é finito e limitado em função de $\left[\gamma_{s}(G): \gamma_{s}(G) \cap Z_{t}(G)\right]$.

Demonstração. Por comodidade denotamos $\bar{G}=G / Z_{t-1}(G)$ e $n=\left[\gamma_{s}(G): \gamma_{s}(G) \cap Z_{t}(G)\right]$. Observe que, pela fórmula (3.1.3), temos $\left[\gamma_{s}(\bar{G}): \gamma_{s}(\bar{G}) \cap Z(\bar{G})\right]=n$. Aplicando a Proposição 3.1.1 ao quociente $\bar{G}$, temos que $\left[\bar{G}: C_{\bar{G}}\left(\gamma_{s}(\bar{G})\right)\right]$ é finito e limitado em função de $n$. Pela Proposição 1.6.5, $\left[\bar{G}: C_{\bar{G}}\left(\gamma_{s}(\bar{G})\right)\right]=\left[G: C_{G}\left(\gamma_{s}(\bar{G})\right)\right]$. Como $\gamma_{s}(\bar{G})$ é isomorfo a $\gamma_{s}(G) /\left(\gamma_{s}(G) \cap Z_{t-1}(G)\right)$, o resultado segue.

A última parte da demonstração do Teorema A segue, em linhas gerais, as mesmas ideias da demostração do Teorema 2.5.4 de Hall. Lembramos que na demonstração do Teorema de Hall o $C_{G}\left(\gamma_{i+1}(G)\right)$ possui um papel fundamental. Este centralizador tem índice finito em $G$ e é tal que $\left[C_{G}\left(\gamma_{i+1}(G)\right){ }_{s} G, C_{G}\left(\gamma_{i+1}(G)\right)\right] \leq Z_{2 i-s-1}(G)$, para todo $s \geq 0$, com a convenção de que $Z_{j}(G)=1$ para $j<0$.

A chave para a generalização do resultado de Hall é mostrar que, aqui também, é possível definir um subgrupo $C$ de índice finito em $G$ tal que $\left[C,{ }_{s} G, C\right] \leq Z_{2 i-s-1}(G)$, para todo $s \geq 0$. No próximo lema, veremos a definição do subgrupo $C$ e mostraremos que ele possui a segunda propriedade enunciada acima.

Lema 3.1.5. Sejam $G$ um grupo e $i \geq 1$. Para $0 \leq j<i$, defina $C_{j}$, o centralizador em $G$ de $\gamma_{i+j+1}(G) /\left(\gamma_{i+j+1}(G) \cap Z_{i-j-1}(G)\right)$. Seja $C=\bigcap_{0 \leq j<i} C_{j}$. Então $\left[C,{ }_{s} G, C\right] \leq Z_{2 i-s-1}(G)$, para todo $s \geq 0$. 
Demonstração. Pela Proposição 1.6.4 $C_{j}$ é normal em $G$, pois o grupo quociente $\gamma_{i+j+1}(G) /\left(\gamma_{i+j+1}(G) \cap Z_{i-j-1}(G)\right)$ é um subgrupo normal do quociente $G /\left(\gamma_{i+j+1}(G) \cap Z_{i-j-1}(G)\right)$. Logo, $C$ é normal em G. Assim, pela Proposição 1.2.5, o comutador $\left[C,_{k} G\right]$ também é normal em $G$, para todo $k \geq 0$.

Primeiro mostraremos que

$$
\left[\left[C,{ }_{k} G\right], \gamma_{r}(G)\right] \leq Z_{2 i-r-k}(G), \quad \forall k \geq 0 \text { e } r \geq i+1
$$

Argumentaremos por indução sobre $k$. Pela definição de $C$, temos $\left[C, \gamma_{r}(G)\right] \leq Z_{2 i-r}(G)$, para todo $r \geq i+1$, e, portanto a afirmação vale para $k=0$. Agora assuma que a afirmação (3.1.4) vale para $k$. Note que, $\left[\gamma_{r}(G), G,\left[C,,_{k} G\right]\right]=\left[\gamma_{r+1}(G),\left[C,_{k} G\right]\right] \leq Z_{2 i-r-k-1}(G)$. Além disso, $\left[\left[C,{ }_{k} G\right], \gamma_{r}(G), G\right] \leq\left[Z_{2 i-k-r}(G), G\right] \leq Z_{2 i-k-r-1}(G)$. Pelo Lema 1.3.5, $\left[\left[C,_{k+1} G\right], \gamma_{r}(G)\right]=\left[\left[C,,_{k} G\right], G, \gamma_{r}(G)\right] \leq Z_{2 i-k-r-1}(G)$. Isto mostra que a afirmação (3.1.4) vale para $k+1$ e conclui a indução.

Agora vamos mostrar que $\left[C,{ }_{s} G, C,,_{2 i-s-1} G\right]=1$, para todo $0 \leq$ $s \leq 2 i-1$. Aplicando o Lema 2.5.1 com $M=\left[C,_{s} G\right]$ e $N=C$, temos

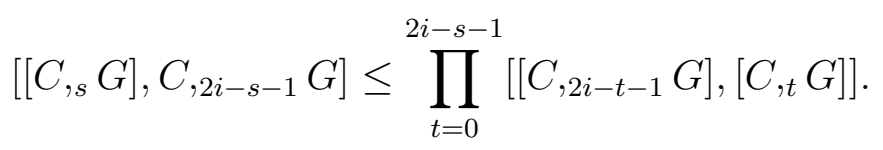

Considere os dois casos: $0 \leq t \leq i-1$ ou $i \leq t \leq 2 i-s-1$. Em ambos os casos vamos mostrar que o fator $\left[[C, 2 i-t-1, G],\left[C,{ }_{t} G\right]\right]$ do produto em (3.1.5) é trivial.

Se $t \leq i-1$ então $2 i-t \geq i+1$. Pela afirmação (3.1.4), temos que

$$
\left[[C, 2 i-t-1=[],[C, t, G]] \leq\left[\gamma_{2 i-t}(G),\left[C,{ }_{t} G\right]\right]=1\right.
$$


Se $i \leq t$ então $t+1 \geq i+1$. Novamente, de (3.1.4) segue que

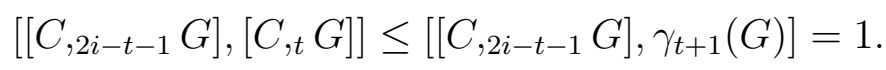

Portanto, todos os fatores do produto em (3.1.5) são triviais e, por consequência, $\left[C,{ }_{s} G, C, 2 i-s-1 G\right]=1$, para todo $0 \leq s \leq 2 i-1$, como queríamos. Segue da Proposição 1.3.3 que $\left[C,{ }_{s} G, C\right] \leq Z_{2 i-s-1}(G)$, para todo $0 \leq s \leq 2 i-1$. Para completar a demonstração falta verificar essa desigualdade para $s \geq 2 i$. Como $C$ é normal em $G$, se $s \geq 2 i$ temos que $\left[C,{ }_{s} G, C\right] \leq\left[C,,_{2 i-1} G, C\right] \leq Z_{0}(G)=1$. Isto completa a demonstração.

Na demonstração do Teorema de Hall, como $\gamma_{i+1}(G)$ é normal e finito, o centralizador $C=C_{G}\left(\gamma_{i+1}(G)\right)$ tem índice finito em $G$. Aqui, apenas com a hipótese de que $\gamma_{i+1}(G) /\left(\gamma_{i+1}(G) \cap Z_{i}(G)\right)$ é finito, é mais difícil mostrar que $C=C_{1} \cap \cdots \cap C_{i-1}$ tem índice finito em $G$. Será a definição mais sofisticada do subgrupo $C$, usada para provar o Teorema A, que vai garantir que $C$ tem índice finito em $G$. A demonstração desse fato é fundamentada pela Proposição 3.1.1 e seus corolários, como veremos a seguir. Para a comodidade do leitor, enunciamos novamente o Teorema A.

Teorema A. Sejam um grupo $G$ e um inteiro $i \geq 0$ tais que $\left[\gamma_{i+1}(G): \gamma_{i+1}(G) \cap Z_{i}(G)\right]$ é finito. Então $\left[G: Z_{2 i}(G)\right]$ é finito e limitado em função de $\left[\gamma_{i+1}(G): \gamma_{i+1}(G) \cap Z_{i}(G)\right]$.

DemonstraÇÃo. Para cada $0 \leq j<i$, chamemos de $C_{j}$ o centralizador em $G$ de $\gamma_{i+j+1}(G) /\left(\gamma_{i+j+1}(G) \cap Z_{i-j-1}(G)\right)$ e seja $C=\bigcap_{0 \leq j<i} C_{j}$ como fizemos no Lema 3.1.5. 
Primeiro mostramos que $[G: C]$ é finito e limitado em função de $n=\left[\gamma_{i+1}(G): \gamma_{i+1}(G) \cap Z_{i}(G)\right]$. Pelo Teorema B, para todo $0 \leq j<i$, temos que o índice $\left[\gamma_{i+j+1}(G): \gamma_{i+j+1}(G) \cap Z_{i-j}(G)\right]$ é finito e limitado em função de $n$. Segue do Corolário 3.1.4, com $s=i+j+1$ e $t=i-j$, que o centralizador em $G$ de $\gamma_{i+j+1}(G) /\left(\gamma_{i+j+1}(G) \cap Z_{i-j-1}(G)\right)$ tem índice finito em $G$, sendo esse índice limitado em função de $n$. Isto é, $\left[G: C_{j}\right]$ é finito e limitado em função de $n$, para todo $0 \leq j<i$. Portanto $[G: C]$ é finito e limitado em função de $n$, pela Proposição 1.0.2.

Agora, para cada $0 \leq s \leq i$, defina

$$
Q_{s}=\frac{\left[C,{ }_{s} G\right]}{\left[C,{ }_{s} G\right] \cap Z_{2 i-s}(G)} .
$$

Vamos mostrar, por indução reversa sobre $s$, que esses quocientes são finitos, com ordens limitadas em função de $n$. Em particular, a ordem de $Q_{0}=C /\left(C \cap Z_{2 i}(G)\right)$ é limitada em função de $n$ e, assim,

$$
\left[G: Z_{2 i}(G)\right] \leq\left[G: C \cap Z_{2 i}(G)\right]=[G: C] \cdot\left[C: C \cap Z_{2 i}(G)\right]<\infty .
$$

Portanto $\left[G: Z_{2 i}(G)\right]$ é finito e limitado em função de $n$, o que prova o teorema.

Façamos a indução. Primeiro notamos que $Q_{i}$ é isomorfo ao quociente $\left[C,_{i} G\right] Z_{i}(G) / Z_{i}(G) \leq \gamma_{i+1}(G) Z_{i}(G) / Z_{i}(G)$, pois $\left[C,_{i} G\right] \leq \gamma_{i+1}(G)$. Como $\gamma_{i+1}(G) Z_{i}(G) / Z_{i}(G)$ é isomorfo a $\gamma_{i+1}(G) /\left(\gamma_{i+1}(G) \cap Z_{i}(G)\right)$, que tem ordem $n$ por hipótese, segue que $\left|Q_{i}\right| \leq n$. Agora, assumindo que $Q_{s+1}=\left[C,_{s+1} G\right] /\left(\left[C,_{s+1} G\right] \cap Z_{2 i-s-1}(G)\right)$ é finito de ordem limitada em função de $n$, vamos mostrar que $Q_{s}$ é finito com ordem limitada em função de $n$. 
Nomeamos $W_{s}=\left[C_{s_{s+1}} G\right] \cap Z_{2 i-s-1}(G)$. Como $C$ tem índice finito em $G$, existem $g_{1}, \ldots, g_{m} \in G$ tais que $G=\left\langle g_{1}, \ldots, g_{m}, C\right\rangle$, onde $m \leq[G: C]$, que é limitado em função de $n$. Defina os grupos $U=$ $\left\langle g_{1}, \ldots, g_{m}\right\rangle$ e $H_{s}=C_{\left[C,{ }_{s} G\right]}\left(U W_{s} / W_{s}\right)$. Notamos que $\left[\left[C,{ }_{s} G\right], U\right] W_{s} / W_{s}$ é subgrupo de $\left[C,_{s+1} G\right] W_{s} / W_{s}=Q_{s+1}$. Como a ordem de $Q_{s+1}$ e o número de geradores de $U$ são limitados em função de $n$, do Corolário 1.6.7 segue que o índice de $H_{s}$ em $\left[C,{ }_{s} G\right]$ é finito e limitado em função de $n$. Vamos mostrar agora que $H_{s} \leq\left[C,_{s} G\right] \cap Z_{2 i-s}(G)$ e disso podemos concluir que $Q_{s}$ é finito de ordem limitada em função de $n$.

Vejamos que, de fato, isso acontece. Por definição, $H_{s} \leq\left[C,,_{s} G\right]$. Assim, basta mostrar que $H_{s} \leq Z_{2 i-s}(G)$. Note que, pela definição de $H_{s}$, tem-se que $\left[H_{s}, U\right] \leq W_{s} \leq Z_{2 i-s-1}(G)$. Por outro lado, temos que $\left[H_{s}, C\right] \leq\left[C,{ }_{s} G, C\right] \leq Z_{2 i-s-1}(G)$, pelo Lema 3.1.5. Logo, pela Proposição 1.2.8, temos $\left[H_{s}, G\right]=\left[H_{s}, U C\right] \leq Z_{2 i-s-1}(G)$ e, assim, $H_{s} \leq Z_{2 i-s}(G)$, como queríamos. Isto termina a indução e a demonstração.

Comparando a demonstração do teorema acima com a do Teorema 2.5.4 de Hall, notamos que a estrutura é a mesma. Ambas usam, da mesma maneira, um subgrupo $C \leq G$, embora a definição desse subgrupo se altere de um teorema para outro, como já observamos. Os quocientes $Q_{s}$ têm a mesma definição a partir do subgrupo $C$.

Em relação aos subgrupos $H_{s}$, que estão presentes em ambas as provas, embora tenham sido definidos de maneira diferente, na verdade as definições coincidem. Isto porque no Teorema A definimos $H_{s}=C_{\left[C,{ }_{s} G\right]}\left(\left\langle g_{1}, \ldots, g_{m}\right\rangle W_{s} / W_{s}\right)=\bigcap_{k=1}^{m} C_{\left[C,{ }_{s} G\right]}\left(g_{k} W_{s}\right)$ enquanto no Teorema de Hall definimos $H_{s}=\bigcap_{k=1}^{r} \operatorname{Ker}\left(\varphi_{g_{k}}\right)$. Agora, se lembrarmos da definição do homomorfismo $\varphi_{g_{k}}$ dada no Teorema de Hall, vemos que 
vale a identidade $\operatorname{Ker}\left(\varphi_{g_{k}}\right)=C_{[C, s G]}\left(g_{k} W_{s}\right)$, para cada $1 \leq k \leq r$, onde $W_{s}=\left[C,_{s+1} G\right] \cap Z_{2 i-s-1}(G)$. Portanto, as definições de $H_{s}$ coincidem, guardadas as diferenças entre os subgrupos $C$ em cada caso.

Terminamos esta seção com algumas questões que surgem naturalmente com respeito ao Teorema A:

(a) Se a hipótese mais fraca de que $\left[\gamma_{i+1}(G): \gamma_{i+1}(G) \cap Z_{i+1}(G)\right]$ é finito vale, ainda é possível mostrar que $\left[G: Z_{2 i}(G)\right]$ é finito?

(b) Seja G um grupo finitamente gerado. O Teorema 2.4.2 garante que se $\gamma_{i+1}(G)$ é finito então $\left[G: Z_{i}(G)\right]$ é finito. É possível mostrar que $\left[G: Z_{i}(G)\right]$ é finito sob a hipótese mais fraca do Teorema $A$ de que $\left[\gamma_{i+1}(G): \gamma_{i+1}(G) \cap Z_{i}(G)\right]$ é finito?

Ambas as perguntas (a) e (b) tem resposta negativa, como mostra o exemplo a seguir retirado de [7, página 426].

Dado um qualquer inteiro $n \geq 1$, considere o produto semidireto $G=G(n)=N \rtimes\langle a\rangle$, onde $a$ tem ordem infinita, $N=N(n)=$ $\left\langle x_{1}, \ldots, x_{n} \mid\left[x_{i}, x_{j}\right]=1\right\rangle$ é o grupo abeliano livre de posto $n$, e $a$ age sobre $N$ da seguinte forma: $x_{n}^{a}=x_{n}$ e $x_{j}^{a}=x_{j} x_{j+1}$, para $1 \leq j \leq n-1$. Ou seja, $\left[x_{n}, a\right]=1$ e $\left[x_{j}, a\right]=x_{j+1}$, para $1 \leq j \leq n-1$.

Dado $x \in N$, temos que $x=x_{1}^{k_{1}} \cdots x_{n}^{k_{n}}$, com $k_{1}, \ldots, k_{n}$ inteiros. Então $[x, a]=x^{-1} x^{a}=\left(x_{1}^{k_{1}} \cdots x_{n}^{k_{n}}\right)^{-1}\left(x_{1}^{a}\right)^{k_{1}} \cdots\left(x_{n}^{a}\right)^{k_{n}}$ e, como $N$ é abeliano e normal em $G$, temos que $[x, a]=\left(x_{1}^{-1} x_{1}^{a}\right)^{k_{1}} \cdots\left(x_{n}^{-1} x_{n}^{a}\right)^{k_{n}}=$ $\left[x_{1}, a\right]^{k_{1}} \cdots\left[x_{n}, a\right]^{k_{n}}$. Logo, usando as relações que definem $G$, temos

$$
[x, a]=\left[x_{1}^{k_{1}} \cdots x_{n}^{k_{n}}, a\right]=x_{2}^{k_{1}} \cdots x_{n}^{k_{n-1}} .
$$

Queremos mostrar que $G^{\prime}=\left\langle x_{2}, \ldots, x_{n}\right\rangle$. Por um lado, é fácil ver que $\left\langle x_{2}, \ldots, x_{n}\right\rangle \leq G^{\prime}$, pois $x_{2}=\left[x_{1}, a\right], \ldots, x_{n}=\left[x_{n-1}, a\right]$. Por outro 
lado, a equação (3.1.6) nos assegura que $[x, a] \in\left\langle x_{2}, \ldots, x_{n}\right\rangle$, qualquer que seja $x \in N$. Então, pela identidade (1.2.4), para todo $x \in N$ e todo $r \geq 1$, temos $\left[x, a^{r}\right]=[x, a][x, a]^{a} \cdots[x, a]^{a^{r-1}} \in\left\langle x_{2}, \ldots, x_{n}\right\rangle$, pois $\left\langle x_{2}, \ldots, x_{n}\right\rangle^{a} \leq\left\langle x_{2}, \ldots, x_{n}\right\rangle$. Além disso, pela fórmula (1.2.7), $\left[x, a^{-r}\right]=\left[x,\left(a^{r}\right)^{-1}\right]=\left(\left[x, a^{r}\right]^{-1}\right)^{a^{-r}} \in\left\langle x_{2}, \ldots, x_{n}\right\rangle$. Agora, dados $g, h \in G$ elementos arbitrários, temos $g=x a^{r}$ e $h=y a^{s}$, com $r$ e $s$ inteiros e $x, y \in N$, de forma que, pela identidade $(1.2 .2),[g, h]=$ $\left[x a^{r}, y a^{s}\right]=\left[x a^{r}, a^{s}\right]\left[x a^{r}, y\right]^{a^{s}}$. Como $N$ e $\langle a\rangle$ são abelianos, segue da identidade (1.2.1) que $[g, h]=\left[x, a^{s}\right]^{a^{r}}\left[a^{r}, y\right]^{a^{s}}=\left[x, a^{s}\right]^{a^{r}}\left(\left[y, a^{r}\right]^{-1}\right)^{a^{s}}$. Isto mostra que $[g, h] \in\left\langle x_{2}, \ldots, x_{n}\right\rangle$, para quaisquer $g, h \in G$, e por consequência $G^{\prime}=\left\langle x_{2}, \ldots, x_{n}\right\rangle$, como queríamos.

Em geral, para $2 \leq k \leq n$, temos $\gamma_{k}(G)=\left\langle x_{k}, \ldots, x_{n}\right\rangle$. Mostraremos isso por indução sobre $k$. O caso $k=2$ foi estabelecido logo acima. Suponha, por indução, que $\gamma_{k}(G)=\left\langle x_{k}, \ldots, x_{n}\right\rangle$. Dados elementos arbitrários $y \in \gamma_{k}(G)$ e $g \in G$, segue da hipótese de indução que o elemento $y=x_{k}^{\alpha_{k}} \cdots x_{n}^{\alpha_{n}}$, com $\alpha_{k}, \ldots, \alpha_{n}$ inteiros. Das relações que definem $G$ deduzimos que $[y, a]=\left[x_{k}^{\alpha_{k}} \cdots x_{n}^{\alpha_{n}}, a\right]=x_{k+1}^{\alpha_{k}} \cdots x_{n}^{\alpha_{n-1}}$ e, portanto, $[y, a] \in\left\langle x_{k+1}, \ldots, x_{n}\right\rangle$. Logo, da equação (1.2.7) segue que $\left[y, a^{-1}\right]=\left([y, a]^{-1}\right)^{a^{-1}} \in\left\langle x_{k+1}, \ldots, x_{n}\right\rangle$. Como $G=N \rtimes\langle a\rangle$, podemos escrever $g=x a^{r}$, com $x \in N$ e $r$ inteiro. Assim, usando a fórmula (1.2.2) e lembrando que $N$ é abeliano, temos $[y, g]=\left[y, x a^{r}\right]=\left[y, a^{r}\right]$. Portanto, $[y, g] \in\left\langle x_{k+1}, \ldots, x_{n}\right\rangle$, pela fórmula (1.2.4). Isto mostra que $\gamma_{k+1}(G) \leq\left\langle x_{k+1}, \ldots, x_{n}\right\rangle$. A inclusão contrária segue do fato de que $x_{k+1}=\left[x_{1, k} a\right], \ldots, x_{n}=\left[x_{n-k, k} a\right]$ todos pertencem à $\gamma_{k+1}(G)$. Portanto, $\gamma_{k+1}(G)=\left\langle x_{k+1}, \ldots, x_{n}\right\rangle$, o que conclui a indução.

Em particular, mostramos que $\gamma_{n}(G)=\left\langle x_{n}\right\rangle \leq Z(G)$ e, portanto, $G$ é nilpotente de classe $n$. Consequentemente, $Z_{n}(G)=G$. 
Queremos mostrar agora que $Z(G)=\left\langle x_{n}\right\rangle$. Seja $g \in Z(G)$ um elemento arbitrário. Suponha que $g=x a^{r}$, com $x \in N$ e $r \geq 1$. Como $g \in Z(G)$, devemos ter $1=\left[x_{n-1}, g\right]=\left[x_{n-1}, x a^{r}\right]=\left[x_{n-1}, a^{r}\right]$, pela identidade (1.2.2). Agora, pela identidade (1.2.4), temos que $\left[x_{n-1}, a^{r}\right]=\left[x_{n-1}, a\right]\left[x_{n-1}, a\right]^{a} \cdots\left[x_{n-1}, a\right]^{a^{r-1}}=x_{n} x_{n}^{a} \cdots x_{n}^{a^{r-1}}=x_{n}^{r}$, pelas relações que definem $G$. Logo, $x_{n}^{r}=1$, que é uma contradição com o fato de $N$ ser abeliano livre. Agora, suponha que $g=x a^{-r}$, com $x \in N$ e $r \geq 1$. Analogamente, devemos ter $1=\left[x_{n-1}, a^{-r}\right]=$ $\left(\left[x_{n-1}, a^{r}\right]^{-1}\right)^{a^{-r}}$, pela identidade (1.2.7). Logo, $1=\left[x_{n-1}, a^{r}\right]$, que também é uma contradição, como vimos acima. Dessas duas contradições concluímos que $g=x a^{0}$ com $x \in N$ e, portanto, $g \in N$. Escrevemos $g=x_{1}^{\alpha_{1}} \cdots x_{n}^{\alpha_{n}}$ e, usando a fórmula (3.1.6), temos que $1=[g, a]=x_{2}^{\alpha_{1}} \cdots x_{n}^{\alpha_{n-1}}$, o que implica que $\alpha_{1}=\cdots=\alpha_{n-1}=0$. Portanto $g=x_{n}^{\alpha_{n}}$. Isto mostra que $Z(G) \leq\left\langle x_{n}\right\rangle$ e, assim, $Z(G)=\left\langle x_{n}\right\rangle$, como queríamos.

Em geral, para todo $1 \leq k \leq n-1$, temos $Z_{n-k}(G)=\left\langle x_{k+1}, \ldots, x_{n}\right\rangle$. Pode-se mostrar esse fato, por indução sobre $k$, usando ideias análogas às que usamos acima. Portanto, para $0 \leq k \leq n$,

$$
\gamma_{k+1}(G)=Z_{n-k}(G)
$$

Em particular, temos que $Z_{n-1}(G)=\left\langle x_{2}, \ldots, x_{n}\right\rangle$ e, como $x_{1}$ e $a$ ambos têm ordem infinita, segue que $\left[G: Z_{n-1}(G)\right]$ é infinito.

Seja $i \geq 1 \mathrm{um}$ inteiro fixado. Escolhendo $G=G(2 i+1)$, segue da identidade (3.1.7), que $\gamma_{i+1}(G)=Z_{i+1}(G)$. Então o índice $\left[\gamma_{i+1}(G): \gamma_{i+1}(G) \cap Z_{i+1}(G)\right]=1$, mas $\left[G: Z_{2 i}(G)\right]$ é infinito. Dando uma resposta negativa à pergunta (a), isso mostra que, mesmo para grupos nilpotentes finitamente gerados, a hipótese mais fraca de que 
$\left[\gamma_{i+1}(G): \gamma_{i+1}(G) \cap Z_{i+1}(G)\right]$ é finito não implica que $\left[G: Z_{2 i}(G)\right]$ é finito.

Por outro lado, escolhendo agora $G=G(2 i)$, segue da equação (3.1.7), que $\gamma_{i+1}(G)=Z_{i}(G)$. Então $\left[\gamma_{i+1}(G): \gamma_{i+1}(G) \cap Z_{i}(G)\right]=1$, mas $\left[G: Z_{i}(G)\right]$ é infinito e, de fato, $\left[G: Z_{j}(G)\right]$ é infinito, qualquer que seja $0 \leq j \leq 2 i-1$. Dando uma resposta negativa à pergunta (b), isso mostra que a hipótese do Teorema A de que $\left[\gamma_{i+1}(G): \gamma_{i+1}(G) \cap Z_{i}(G)\right]$ é finito não é suficiente para mostrar que $\left[G: Z_{i}(G)\right]$ é finito, mesmo para grupos (nilpotentes) finitamente gerados. Com essa hipótese, não podemos garantir nem mesmo que $\left[G: Z_{j}(G)\right]$ é finito, para algum inteiro $0 \leq j \leq 2 i-1$.

\subsection{Grupos capable e grupos $i$-capable}

Nesta seção apresentamos os conceitos de grupos capable e grupos i-capable. Depois mostraremos que o Teorema A de Fernández-Alcober e Morigi pode ser interpretado como uma recíproca do Teorema de Baer para a classe dos grupos $i$-capable.

Foi Baer em [1] quem começou o estudo sistemático da seguinte questão: quais condições um grupo deve satisfazer para que seja isomorfo ao quociente central de algum grupo E? Seguindo M. Hall e Senior em [10], chamamos tais grupos de capable. Em linhas gerais, um grupo é capable se for isomorfo ao grupo de automorfismos internos de algum grupo. Mais precisamente, damos a seguinte definição.

Definição 3.2.1. Dizemos que um grupo $G$ é capable se existe um grupo $E$ tal que $G$ é isomorfo a $E / Z(E)$. Ou, equivalentemente, se existe um grupo $E$ tal que $G$ é isomorfo a $\operatorname{Inn}(E)$. 
Observamos que existem grupos que não são capable, como por exemplo os grupos cíclicos não triviais. De fato, se um grupo cíclico não trivial fosse capable, existiria um grupo abeliano cujo grupo de automorfismos internos é não trivial, o que é um absurdo. Assim, ser capable implica em restrições sobre a estrutura do grupo.

O artigo [1] de Baer tem por objetivo principal determinar todos os grupos capable abelianos que são produtos diretos de grupos cíclicos (não necessariamente de uma quantidade finita deles). A proposição a seguir é consequência dos resultados de Baer. Para $n \geq 0$, denotamos por $\mathbb{Z}_{n}$ o grupo cíclico de ordem $n$, onde por convenção $\mathbb{Z}_{0}=\mathbb{Z}$.

Proposição 3.2.2. Seja A um grupo abeliano finitamente gerado escrito como $A=\mathbb{Z}_{n_{1}} \oplus \cdots \oplus \mathbb{Z}_{n_{r}}$, com $n_{j} \mid n_{j+1}$, para todo $1 \leq j \leq r-1$. Então A é capable se, e somente se, $r \geq 2$ e $n_{r}=n_{r-1}$.

Assim, sabemos por exemplo que $\mathbb{Z}_{2} \oplus \mathbb{Z}_{2}$ e $\mathbb{Z}_{3} \oplus \mathbb{Z} \oplus \mathbb{Z}$ são capable enquanto $\mathbb{Z}_{2}$ e $\mathbb{Z}_{3} \oplus \mathbb{Z}_{6}$ não são capable.

Em [13] P. Hall enfatizou que caracterizações de grupos capable são importantes na classificação dos p-grupos. Beyl em [3] e Ellis em [5], usando homologia, fornecem várias condições necessárias e condições suficientes para que certos grupos sejam capable.

Dois artigos relativamente recentes tratam da recíproca do Teorema de Schur para grupos capable. Em [17, Theorem A] Isaacs mostra, para grupos finitos capable, que o índice do centro é limitado por uma função que depende da ordem do subgrupo derivado. Em [24, Corollary 2] Podoski e Szegedy estendem o resultado de Isaacs para grupos capable quaisquer, sem exigir que a ordem do grupo seja finita. A principal importância de [24] é a cota mais apurada, obtida pelos autores, para o índice do centro em função da ordem do subgrupo derivado. Pois, 
essencialmente, a recíproca do Teorema de Schur para grupos capable já havia sido estabelecida por Macdonald em [20, Corollary 2]. (Obviamente, Macdonald não usava o termo capable, pois esse viria a ser definido apenas dois anos depois por M. Hall).

Outros resultados sobre grupos capable aparecem em [17] e [15]. Se $G$ é um $p$-grupo capable finito com $\left|G^{\prime}\right|=p$ então $[G: Z(G)]=p^{2}$ (veja $[\mathbf{1 7}$, p. 2853] e suas referências). Heineken $[\mathbf{1 5}$, p. 248] mostra que se $G$ é um grupo capable finito tal que $G^{\prime}$ é isomorfo ao $p$-grupo abeliano elementar de ordem $p^{2}$ então $[G: Z(G)]<p^{6}$. Heineken construiu, para cada primo $p>2$ e cada $n \geq 1$, um grupo capable finito $G$ tal que $G^{\prime}$ é um $p$-grupo abeliano elementar de ordem $p^{n} \operatorname{com}[G: Z(G)]=p^{2 n+\left(\begin{array}{l}n \\ 2\end{array}\right)}$ e $\left|G^{\prime}\right|=|Z(G)|($ veja [15, Proposition 3]).

O resultado abaixo, que é uma recíproca do Teorema de Schur, ilustra o quanto a definição de grupo capable é pertinente no contexto desta dissertação.

Teorema 3.2.3. [24, Corollary 2] Seja $G$ um grupo capable. Se $G^{\prime}$ é finito então $[G: Z(G)] \leq\left|G^{\prime}\right|^{2 \log _{2}\left|G^{\prime}\right|}$.

Este teorema fornece mais um critério para decidir se um grupo é capable ou não: se um dado grupo $G$ não satisfaz a recíproca do Teorema de Schur então ele não pode ser capable. No entanto, há grupos que satisfazem a recíproca do Teorema de Schur mas não são capable. Portanto, satisfazer a recíproca do Teorema de Schur não é um critério decisivo, apenas um critério eliminatório. A seguir fornecemos um exemplo de um grupo não capable que satisfaz a recíproca do Teorema de Schur. 
Exemplo 3.2.4. Considere o grupo $G=C_{p^{\infty}} \times A_{5}$, o produto direto do $p$-grupo de Prüfer pelo grupo alternado de grau 5. Lembramos que $C_{p^{\infty}}=\left\langle x_{1}, x_{2}, \ldots \mid x_{1}^{p}=1, x_{2}^{p}=x_{1}, x_{3}^{p}=x_{2}, \ldots\right\rangle$ e que $C_{p^{\infty}}$ é um grupo divisível. Lembramos ainda que $A_{5}$ é um grupo simples. Note que, $G^{\prime}=A_{5}$, pois $C_{p^{\infty}}$ é abeliano, e $Z(G)=C_{p^{\infty}}$, pois $Z\left(A_{5}\right)=1$. Então $G^{\prime}$ e $[G: Z(G)]$ são finitos. Aplicando [5, Proposition 2 (ii)], vemos que $G$ é non-capable, pois $G^{a b}=G / G^{\prime} \cong C_{p^{\infty}}$ é divisível e $Z(G)=C_{p^{\infty}}$ contém um elemento não trivial de ordem finita. Este exemplo mostra que nem todo grupo em que $G^{\prime}$ e $[G: Z(G)]$ são finitos é capable.

Generalizando a terminologia de M. Hall e Senior foi proposta, simultaneamente por Burns e Ellis em [4, p. 406] e por Moghaddam e Kayvanfar em [21], a definição de grupo $i$-capable, para $i \geq 1$.

DefiniÇÃo 3.2.5. Seja $i \geq 1$ um inteiro. Dizemos que um grupo $G$ é $i$-capable se existe um grupo $E$ tal que $G$ é isomorfo a $E / Z_{i}(E)$. Ou, recursivamente, dizemos que $G$ é 1 -capable se for capable e, para $i \geq 2$, dizemos que $G$ é $i$-capable se existe um grupo $(i-1)$-capable $K$ tal que $G$ é isomorfo a $\operatorname{Inn}(K)$.

Se um grupo $G$ é $i$-capable, para algum $i \geq 2$, então $G$ é $j$-capable, para todo $1 \leq j \leq i$. Basta notar que, para todo grupo $E$, temos que $E / Z_{i}(E)$ é isomorfo a $\left(E / Z_{i-j}(E)\right) / Z_{j}\left(E / Z_{i-j}(E)\right)$. Logo, se $G$ é isomorfo a $E / Z_{i}(E)$ então $G$ é isomorfo a $H / Z_{j}(H)$, onde $H=E / Z_{i-j}(E)$.

Em [4, Theorem 1.3] Burns e Ellis caracterizam todos os grupos i-capable abelianos finitamente gerados. Enunciamos esse resultado a seguir. 
Proposição 3.2.6. Seja A um grupo abeliano finitamente gerado e $i \geq 1$ um inteiro fixado. Então A é $i$-capable se, e somente se, $A$ é capable.

Em seguida Burns e Ellis [4, Theorem 1.4] provam a seguinte proposição, a qual mostra que a Proposição 3.2.6 não é válida para grupos não abelianos.

Proposição 3.2.7. Existe um grupo capable que não é 2-capable.

Observamos que o grupo usado na demonstração da Proposição 3.2.7 dada em [4] é um 2-grupo finito, sendo em particular um grupo nilpotente.

Concluímos esta seção mostrando que o Teorema A pode ser visto como uma recíproca do Teorema de Baer para grupos $i$-capable.

ProposiçÃo 3.2.8. Seja i um inteiro positivo. São equivalentes:

(1) se $H$ é um grupo tal que $\left[\gamma_{i+1}(H): \gamma_{i+1}(H) \cap Z_{i}(H)\right]$ é finito então o indice $\left[H: Z_{2 i}(H)\right]$ é finito;

(2) se $G$ é um grupo $i$-capable tal que $\gamma_{i+1}(G)$ é finito então o indice $\left[G: Z_{i}(G)\right]$ é finito.

DemonstraçÃo. Vejamos primeiro que (1) implica em (2). Seja $G$ um grupo $i$-capable tal que $\gamma_{i+1}(G)$ é finito. Como $G$ é $i$-capable, existe um grupo $E$ tal que $G$ é isomorfo a $E / Z_{i}(E)$. Assim,

$\left|\gamma_{i+1}(G)\right|=\left|\operatorname{gamma}_{i+1}\left(E / Z_{i}(E)\right)\right|=\left[\gamma_{i+1}(E): \gamma_{i+1}(E) \cap Z_{i}(E)\right]<\infty$

Então, pelo item (1) aplicado ao grupo $E$, temos que $\left[E: Z_{2 i}(E)\right]$ é finito. Como $\left[E: Z_{2 i}(E)\right]=\left[E / Z_{i}(E): Z_{i}\left(E / Z_{i}(E)\right)\right]=\left[G: Z_{i}(G)\right]$, segue que $\left[G: Z_{i}(G)\right]$ é finito. 
Agora mostramos que (2) implica em (1). Seja $H$ um grupo tal que $\left[\gamma_{i+1}(H): \gamma_{i+1}(H) \cap Z_{i}(H)\right]$ é finito. Defina $G=H / Z_{i}(H)$ e note que

$$
\left[\gamma_{i+1}(H): \gamma_{i+1}(H) \cap Z_{i}(H)\right]=\left|\gamma_{i+1}\left(H / Z_{i}(H)\right)\right|=\left|\gamma_{i+1}(G)\right|<\infty
$$

Pelo item (2) aplicado ao grupo $i$-capable $G$ temos que $\left[G: Z_{i}(G)\right]$ é finito. Como $\left[G: Z_{i}(G)\right]=\left[H / Z_{i}(H): Z_{i}\left(H / Z_{i}(H)\right)\right]=\left[H: Z_{2 i}(H)\right]$, segue que $\left[H: Z_{2 i}(H)\right]$ é finito. 


\section{Índice Remissivo}

ação de grupos, 27

cadeia ascendente, 5

centralizador, 11

centro, 11

classe de nilpotência, 24

comprimento derivado, 23

comutador, 10

comutador de peso $n, 10$

condição maximal, 5

conjugado, 10

finito-por-nilpotente, 55

grupo $i$-capable, 82

grupo capable, 79

grupo de expoente finito, 3

grupo finitamente gerado, 3

grupo metabeliano, 23

grupo nilpotente, 24

grupo periódico, 3

grupo residualmente finito, 51

grupo solúvel, 23

homomorfismo transfer, 32

Identidade de Hall-Witt, 17

identidades de comutadores, 10
Lema dos Três Subgrupos, 17

normalizador, 11

p-grupo extra especial, 25

produto central, 48

produto entrelaçado, 48

representação permutacional, 28

série abeliana, 23

série central ascendente, 16

série central descendente, 14

série derivada, 23

subconjunto normal, 12

subgrupo comutador, 11

subgrupo de Frattini, 60

subgrupo derivado, 14

subgrupo maximal, 60

Teorema de Baer, 46

Teorema de Hall, 57

Teorema de

Reidemeister-Schreier, 9

Teorema de Schreier, 8

Teorema de Schur, 39 


\section{Referências Bibliográficas}

[1] R. Baer, Groups with preassigned central and central quotient group, Trans. Amer. Math. Soc., 44 (1938), 387-412.

[2] R. Baer, Representations of groups as quotient groups. II. Minimal central chains of a group, Trans. Amer. Math. Soc., 58 (1945), 348-389.

[3] F. R. Beyl, U. Felgner and P. Schmid, On groups occurring as center factor groups, J. Algebra, 61 (1979), 161-177.

[4] J. Burns and G. Ellis, On the nilpotent multipliers of a group, Math. Z., 226 (1997) 405-428.

[5] G. Ellis, On the capability of groups, Proc. Edinburgh Math. Soc., 41 (1998), 487-495.

[6] G. A. Fernández-Alcober and A. Moretó, A finiteness condition on normal subgroups of nilpotent groups, J. Group Theory 5 (2002), 301-315.

[7] G. A. Fernández-Alcober and M. Morigi, Generalizing a theorem of P. Hall on finite-by-nilpotent groups, Proc. Amer. Math. Soc., 137 (2009) n.2, 425-429.

[8] D. Gorenstein, Finite Groups. 2nd Ed., Chelsea, New York, 1980.

[9] Z. Halasi and K. Podoski, Bounds in groups with trivial Frattini subgroup, J. Algebra, 319 n. 3 (2008), 893-896.

[10] M. Hall and J. K. Senior, The groups of order $2^{n}(n \leq 6)$, MacMillan, New York, 1964.

[11] P. Hall, Finite-by-nilpotent groups, Proc. Camb. Math. Soc., 52 (1956), 611616.

[12] P. Hall and G. Higman, On the $p$-lenght of $p$-soluble groups, Proc. London Math. Soc. (3) 6 (1956) 1-42.

[13] P. Hall, The classification of prime-power groups, J. Reine Angew. Math., (Crelle) 182 (1940), 130-141. 
[14] R. Hatamian, M. Hassanzadeh and S. Kayvanfar, A converse of Baer's theorem, Ricerche Mat., 63 (2014), 183-187.

[15] H. Heineken, Nilpotent groups of class two that can appear as central quotient groups, Rend. Sem. Mat. Univ. Padova, 84 (1990), 241-248.

[16] N. S. Hekster, On the structure of n-isoclinism classes of groups, J. Pure Appl. Algebra, 40 (1986) 63-85.

[17] I. M. Isaacs, Derived subgroups and centers of capable groups, Proc. Amer. Math. Soc., 129 (2001), 2853-2859.

[18] I. M. Isaacs, Finite group theory. Amer. Math. Soc., 2008.

[19] I. Kaplansky, An introduction to differential Algebra. Hermann, 1957.

[20] I. D. Macdonald, On central series, Proc. Edinburgh Math. Soc., 13 (1962) $175-178$.

[21] M. Moghaddam and S. Kayvanfar, A new notion derived from varieties of groups, Algebra Colloq., 4 (1997) 1-11.

[22] B. H. Neumann, Groups with finite classes of conjugate elements, Proc. London Math. Soc., 3 n. 1 (1951), 178-187.

[23] P. Niroomand, The converse of Schur's theorem, Arch. Math., (Basel), 94 n. 5 (2010) 401-403.

[24] K. Podoski and B. Szegedy, Bounds for the index of the centre in capable groups, Proc. Amer. Math. Soc., 133 (2005) n. 12, 3441-3445.

[25] D. J. S. Robinson, A course in the theory of groups. 2nd Ed., Springer-Verlag, 1996.

[26] D. J. S. Robinson, Finiteness conditions and generalized soluble groups. Part 1, Springer-Verlag, 1972.

[27] M. Rosenlicht, On a result of Baer, Proc. Amer. Math. Soc., 13 (1962), 99-101.

[28] J. J. Rotman, Advanced Modern Algebra. Prentice Hall, 2002.

[29] J. J. Rotman, An introduction to the theory of groups. 4th Edition, SpringerVerlag, 1994.

[30] J. Wiegold, Multiplicators and groups with finite central factor-groups, Math. Z., 89 (1965), 345-347. 